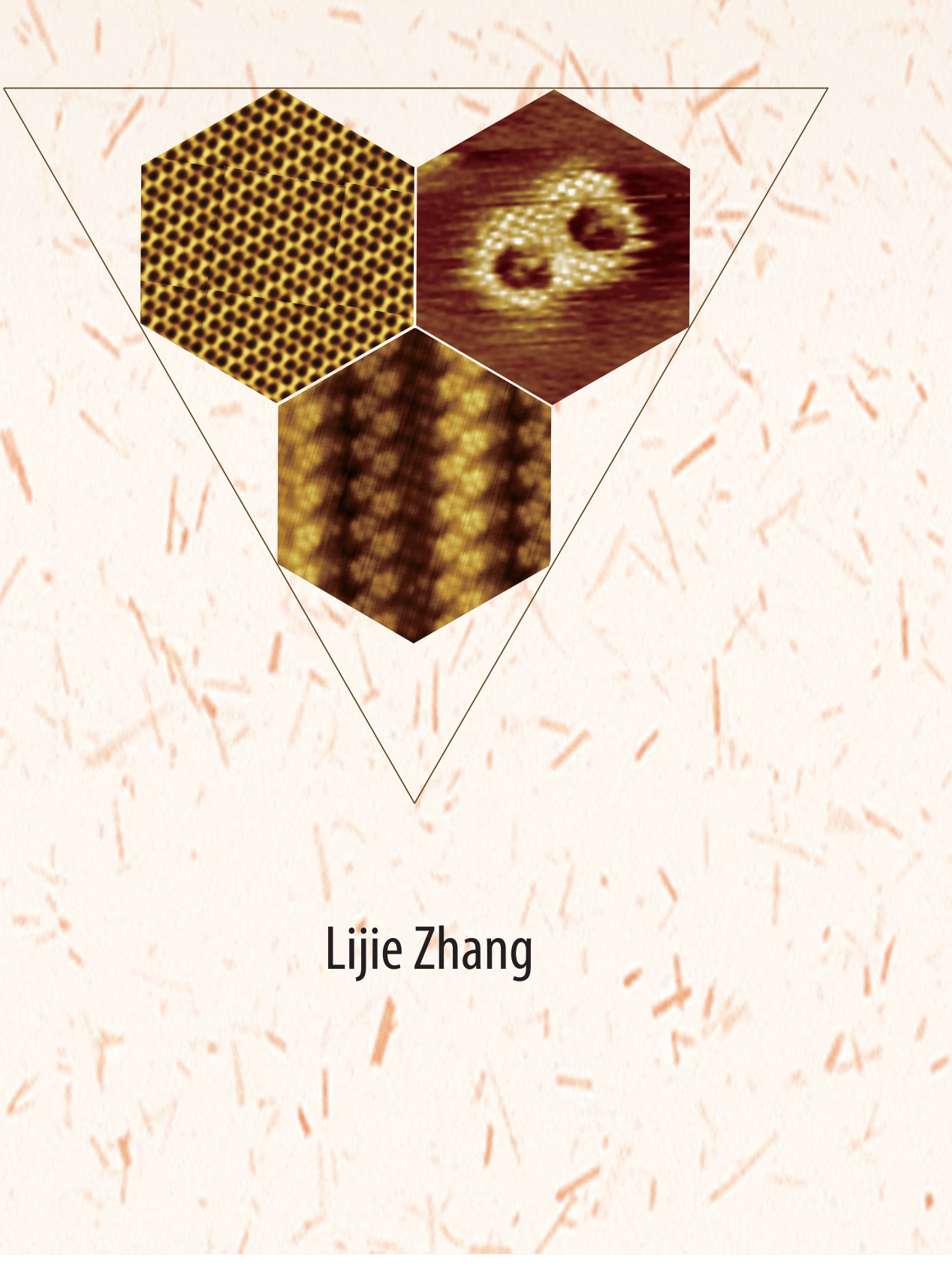




\section{Structural and electronic properties of one- and two- dimensional systems studied by scanning tunneling microscopy}

Lijie Zhang 


\section{Composition of graduation committee:}

Chairman and secretary: $\quad$ Prof.dr.ir. J.W.M. Hilgenkamp

Supervisor: $\quad$ Prof.dr.ir. H.J.W. Zandvliet

Co-supervisor: Dr. A. van Houselt

Members: $\quad$ Prof.dr.ir B. Poelsema

Prof.dr.ir. M. Huijben

Prof.dr. G. Palasantzas

Prof.dr.ir. N. Oncel

The work described in this thesis was carried out in the Physics of Interfaces and Nanomaterials group, MESA+ Institute for Nanotechnology, University of Twente, the Netherlands. This work is funded by the China Scholarship Council.

Published by Physics of Interfaces and Nanomaterials, University of Twente.

Cover Design: Lijie Zhang

Printed by: Gildeprint drukkerijen

CL. Zhang, 2017, Enschede, the Netherlands

No part of this publication may be stored in a retrieval system, transmitted or reproduced in any way, including but not limited to photocopy, photograph, magnetic or other record, without prior agreement and written permission of the publisher.

ISBN: 978-90-365-4347-7

DOI: $10.3990 / 1.9789036543477$ 


\title{
STRUCTURAL AND ELECTRONIC PROPERTIES OF ONE- AND TWO- DIMENSIONAL SYSTEMS STUDIED BY SCANNING TUNNELING MICROSCOPY
}

\author{
DISSERTATION \\ to obtain \\ the degree of doctor at the University of Twente, \\ on the authority of the rector magnificus, \\ Prof. Dr. T.T.M. Palstra, \\ on account of the decision of the graduation committee, \\ to be publicly defended \\ on 8 September 2017 at 16.45
}

by

Lijie Zhang

born on 18 September 1984

in Hebei, China 
This dissertation has been approved by the supervisor:

Prof.dr.ir. H.J.W. Zandvliet

and the co-supervisor:

Dr. A. van Houselt 


\section{Contents}

1 Introduction 1

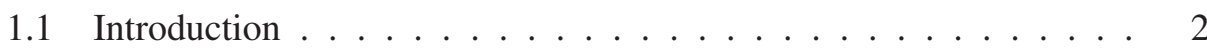

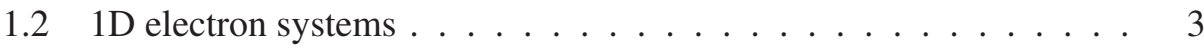

1.2.1 Free electron model . . . . . . . . . . . . . . . . . 3

1.2.2 Nearly free electron model . . . . . . . . . . . . . . . 4

1.3 2D Dirac materials . . . . . . . . . . . . . . 5

1.3.1 Graphene ................... 5

1.3.2 Germanene . . . . . . . . . . . . . . . . 10

1.4 Scope and outline . . . . . . . . . . . . . . . . . . 11

2 Experimental 17

2.1 The basic principle of STM . . . . . . . . . . . . 18

2.1 .1 The tunneling effect $\ldots \ldots \ldots \ldots$

2.1 .2 Tunneling current . . . . . . . . . . . . . . . 20

2.2 Scanning tunneling spectroscopy $\ldots \ldots \ldots \ldots \ldots \ldots \ldots$

2.3 Omicron STM-1 . . . . . . . . . . . . . . . . . . . . . 23

$3 \mathrm{Ge}(110)$ surface and Au-induced nanowires 27

3.1 Introduction . . . . . . . . . . . . . . . . . 28

3.2 Experimental details . . . . . . . . . . . . . . . . . . . . . . 29

3.3 Properties of the bare $\mathrm{Ge}(110)$ surface $\ldots \ldots \ldots . \ldots 30$ 
3.4 Au-induced nanowires $\ldots \ldots \ldots \ldots$

3.5 Conclusions . . . . . . . . . . . . . . . . . . . . 36

4 Pt-induced nanowires on $\mathrm{Ge}(110)$

4.1 Introduction . . . . . . . . . . . . . . . . . . . . . 44

4.2 Experimental details $\ldots \ldots \ldots \ldots \ldots$. . . . . . . . . 45

4.3 Nanowire formation . . . . . . . . . . . . . . . . . . . . . 46

4.4 3D clusters . . . . . . . . . . . . . . . . . . . . . . . . . . 49

4.5 Conclusions . . . . . . . . . . . . . . . . . . . 50

5 Germanene termination of Ge-Pt crystals on $\mathrm{Ge}(110)$

5.1 Introduction . . . . . . . . . . . . . . . . . . 58

5.2 Experimental details $\ldots \ldots \ldots \ldots$. . . . . . . . . 60

5.3 3D Ge-Pt nanocrystals . . . . . . . . . . . . . . . 61

5.4 Spectroscopy . . . . . . . . . . . . . . . . 65

5.5 Conclusions . . . . . . . . . . . . . . . . . . 70

6 Synthesis of germanene on MoS $_{2}$

6.1 Introduction . . . . . . . . . . . . . . 76

6.2 Experimental and calculation details . . . . . . . . . . . 77

6.3 Results and discussion . . . . . . . . . . . . . . 78

$6.3 .1 \quad \mathrm{MoS}_{2} \ldots \ldots \ldots \ldots \ldots \ldots$

6.3.2 Germanene islands . . . . . . . . . . . . . . . . . . . 79

6.3.3 Monolayer germanene . . . . . . . . . . . . . . . 81

6.3.4 Density functional theory calculations . . . . . . . . . 82

6.3.5 Ambient experiments . . . . . . . . . . . . . . 85

6.4 Conclusions . . . . . . . . . . . . . . . . . . . . 85 
Summary

Samenvatting

Publications

99

Acknowledgements 



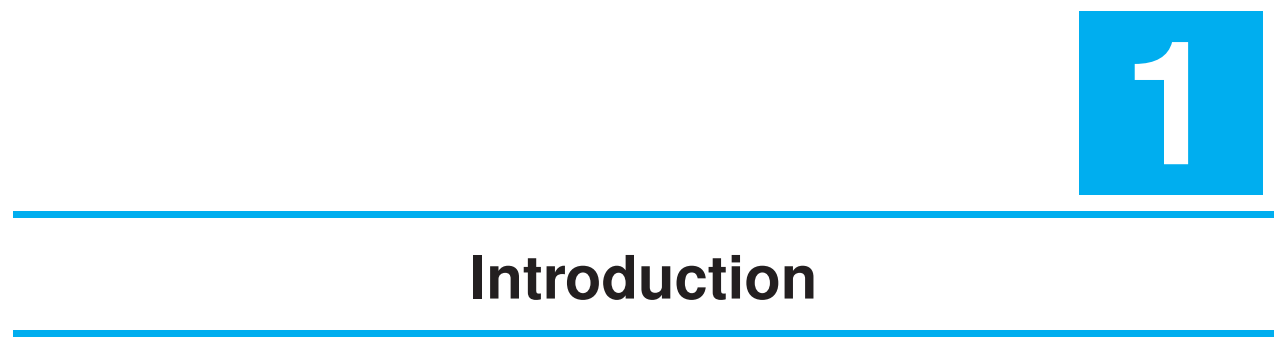

"The eternally incomprehensible thing about the world is its comprehensibility."

- Albert Einstein 


\subsection{Introduction}

The story of materials is the story of human civilization. Technological revolutions have occured quite frequently in modern history especially in the past century. Richard Feynman's lecture: There's plenty of room at the bottom which was held in $1959^{1}$ marked the beginning of the era of nanotechnology. If a structure has at least, in one of its dimensions, a size smaller than $100 \mathrm{~nm}$ one refers to this object as a nanostructure.

The properties of electron systems depend on its dimensions. If electrons are restricted to one-dimensional (1D) systems, interesting exotic phenomena will emerge. The $1 \mathrm{D}$ electron system is often described by the Luttinger liquid theory. ${ }^{2-4}$ The Luttinger liquid theory is a theoretical model to describe interacting electrons in a one-dimensional conductor where the Fermi liquid breaks down. The charactertistic feature of a Luttinger liquid is that the charge and spin degrees of freedom of the electrons are separated: the electron loses its identity and splits up into two quasi-particles: one only carries the spin (spinon) and the other only carries the charge (holon). As an example of Luttinger liquid system we refer to the experiments on carbon nanotubes ${ }^{5-8}$ and quantum wires. ${ }^{9-11}$ However, experimental claims of Luttinger liquid behavior are still under debate. ${ }^{12,13}$ We will not elaborate on the Luttinger liquid system here. Nanowires, atomic chains, synthesized by self-organization of metals on semiconductor surface, are known to be quasi onedimensional systems since 2003, see Gurlu et al. ${ }^{14}$

Graphene, the first real two-dimensional (2D) crystalline material, was isolated successfully in 2004 by Geim and Novoselov, ${ }^{15}$ who were awarded the Nobel Prize in physics in 2010: "for groundbreaking experiments regarding the two-dimensional material graphene". The electrons near the $K$ and $K^{\prime}$ points of the Brillouin zone of graphene behave as relativistic massless particles that are described by the Dirac equation, i.e., the relativistic variant of the Schrödinger equation. The electronic states of graphene near the Dirac points are described by a linear dispersion relation. Here, we will address the basic theory of 1D and 2D electron systems. 


\subsection{D electron systems}

1D electron systems are of great interest because they exhibit exotic physical phenomena, such as quantization of conductance, ${ }^{16}$ a Peierls instability, ${ }^{17}$ spin-and charge-density waves ${ }^{18,19}$ and Luttinger liquid behavior. ${ }^{12,20,21}$ In this paragraph, we will briefly address the theory of $1 \mathrm{D}$ electron systems.

\subsubsection{Free electron model}

The free electron model is the simplest model to explain the behavior of electrons. In this model both electron-electron interactions and electron-phonon interactions are ignored. In a 1D system, we assume that electons are confined to a line segment with length $L$. The eigenfunctions $\left(\Psi_{n}(x)\right)$ and eigenvalues $\left(E_{n}\right)$ can be extracted from the time-independent Schrödinger equation.

$$
-\frac{\hbar^{2}}{2 m} \frac{\partial^{2}}{\partial x^{2}} \Psi_{n}(x)=E_{n} \Psi_{n}(x)
$$

Using the periodic boundary conditions, $\Psi_{n}(x=0)=\Psi_{n}(x=L)$, we find

$$
\begin{aligned}
\Psi_{n}(x) & =C e^{i k x} \\
E_{n} & =\frac{\hbar^{2} k^{2}}{2 m}=\frac{\hbar^{2}}{2 m}\left(\frac{2 \pi n}{L}\right)^{2}
\end{aligned}
$$

with $\mathrm{n}=0, \pm 1, \pm 2, \ldots, \infty$

The energy dispersion curve, $E(k)$, is quadratic in $k$ and the density of states in $k$ space, $D(k)$, is $L / 2 \pi$. The total number of free electrons, $N$, allows us to determine the Fermi wave vector

$$
N=\int_{k=-k_{F}}^{k_{F}} 2 D(k) d k=\frac{2 L}{\pi} k_{F}
$$

where we have assumed that both $N$ and $L$ are very large. The factor 2 in front of the $D(k)$ term arises due to the spin degeneracy. The density of states in energy 
space can easily be derived by setting $2 D(k) d k=D(E) d E$ :

$$
D(E)=\frac{2 D(k)}{d E / d k}=\frac{L / \pi}{\hbar^{2} k / m}=\frac{L}{\pi \hbar} \sqrt{m / 2 E}
$$

For an energy band with its minimum at $E=E_{0}$ the $1 / \sqrt{E}$ term should be replaced by $1 / \sqrt{E-E_{0}}$. Since both positive and negative $k$-values lead to the same energy the total density of states should be multiplied by an additional factor 2 , i.e.

$$
D(E)=\frac{2 L}{\pi \hbar} \sqrt{m / 2\left(E-E_{0}\right)}
$$

Sometimes, the density of states is given per unit length,

$$
D(E)=\frac{2}{\pi \hbar} \sqrt{m / 2\left(E-E_{0}\right)}
$$

As shown above, the density of states of one-dimensional electron gas is proportional to $E^{-1 / 2}$. The singularity at the minimum of the energy, i.e. $E_{0}$, is referred to as a van Hove singularity. ${ }^{22}$

\subsubsection{Nearly free electron model}

The free-electron model has its limits and cannot describe the opening of a band gap. We extend the free-electron model by taking into account the periodic potencial of the lattice,

$$
\left(-\frac{\hbar^{2}}{2 m} \frac{\partial^{2}}{\partial x^{2}}+V(x)\right) \Psi_{n}(x)=E_{n} \Psi_{n}(x)
$$

where the potential is periodic with the lattice constant $a$, i.e. $V(x+a)=V(x)$. It can be expanded in the following Fourier series: $V(x)=\sum_{G} V_{G} e^{i G x}$ where, $G$ is a reciprocal lattice vector $(G=2 \pi m / a, m \in Z)$. Based on the Bloch theorem the solutions of Schrödinger equation have the form:

$$
\psi_{k}(x)=u_{k}(x) e^{i k x}
$$


where $u_{k}(x)$ has the periodicity of the crystal lattice, i.e. $u_{k}(x+a)=u_{k}(x)$. The incorporation of a periodic potential gives rise to forbidden and allowed energy bands due to the opening of bandgaps at the edges and center of the Brillouin zone, $k= \pm \frac{\pi n}{a}$ :

$$
E=\frac{1}{2}\left(E_{k-G}^{0}+E_{k}^{0}\right) \pm \sqrt{\frac{1}{4}\left(E_{k-G}^{0}-E_{k}^{0}\right)^{2}+\left|V_{G}\right|^{2}}
$$

where,

$$
E_{k-G}^{0}=\frac{\hbar^{2}(k-G)^{2}}{2 m}=\frac{\hbar^{2}\left(k-\frac{2 \pi m}{a}\right)^{2}}{2 m}
$$

\subsection{D Dirac materials}

Dirac materials are a class of materials where the electrons behave as massless relativistic particles obeying the Dirac equation rather than the Schrödinger equation. In the vicinity of the Dirac point, the dispersion relation is linear forming a cone-like structure referred to as a Dirac cone. The discovery of graphene, the first real 2D Dirac material, opened the door to a total new research field.

\subsubsection{Graphene}

Graphene, a monolayer of $s p^{2}$ hybridized carbon atoms arranged in hexagonal structure is shown in Figure 1.1. The honeycomb lattice of graphene consists of two sublattices (signed A and B in Figure 1.1) with a lattice constant of $2.46 \AA$, while the carbon-carbon distance is $a=1.42 \AA$. The lattice vectors can be written as,

$$
\begin{aligned}
& \overrightarrow{a_{1}}=\sqrt{3} a(1,0) \\
& \overrightarrow{a_{2}}=\sqrt{3} a\left(\frac{1}{2}, \frac{\sqrt{3}}{2}\right)
\end{aligned}
$$


The reciprocal lattice vectors are,

$$
\begin{aligned}
\overrightarrow{b_{1}} & =\frac{4 \pi}{3 a}\left(\frac{\sqrt{3}}{2},-\frac{1}{2}\right) \\
\overrightarrow{b_{2}} & =\frac{4 \pi}{3 a}(0,1)
\end{aligned}
$$

In the first Brillouin zone there are four high-symmetry points: $\Gamma, M, K$ and $K^{\prime}$

$$
\begin{aligned}
\Gamma & =(0,0) \\
M & =\frac{2 \pi}{3 a}\left(\frac{\sqrt{3}}{2},-\frac{1}{2}\right) \\
K & =\frac{4 \sqrt{3} \pi}{9 a}(1,0) \\
K^{\prime} & =\frac{4 \sqrt{3} \pi}{9 a}\left(\frac{1}{2},-\frac{\sqrt{3}}{2}\right)
\end{aligned}
$$
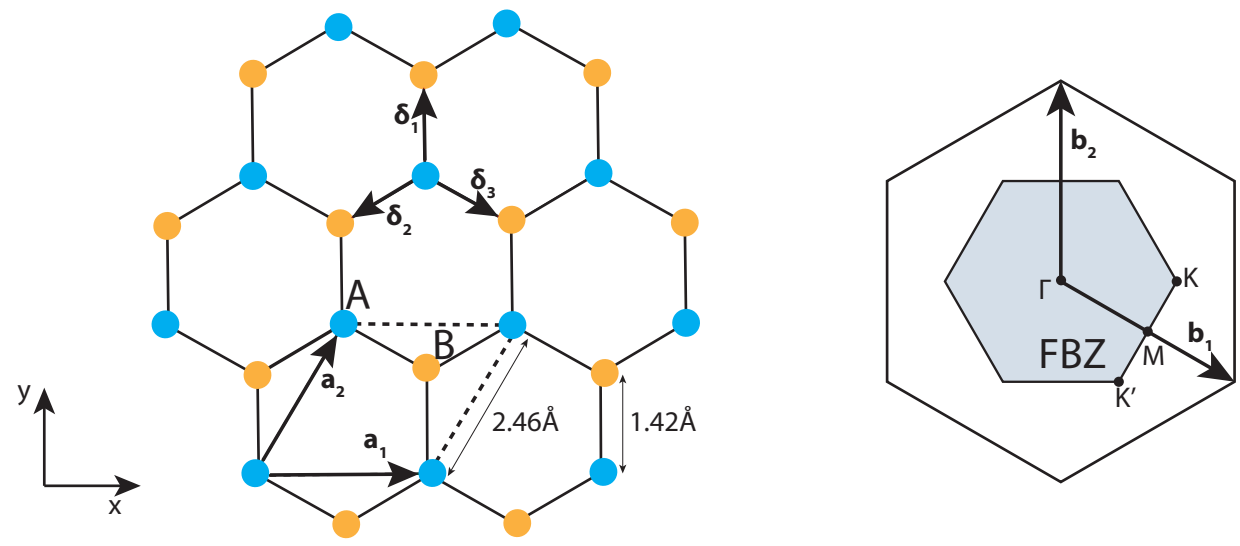

Figure 1.1: Honeycomb structure of graphene consisting of sublattice A (blue) and $\mathrm{B}$ (orange) and its corresponding first Brillouin zone. $\overrightarrow{a_{1}}$ and $\overrightarrow{a_{2}}$ are the lattice unit vectors, $\overrightarrow{\delta_{1}}, \overrightarrow{\delta_{2}}$ and $\overrightarrow{\delta_{3}}$ are the nearest-neighbor vectors. 
The $K$ and $K^{\prime}$ points are referred to as Dirac points. The real space nearest-neighbor vectors are given by,

$$
\begin{aligned}
& \overrightarrow{\delta_{1}}=a(0,1) \\
& \overrightarrow{\delta_{2}}=a\left(-\frac{\sqrt{3}}{2},-\frac{1}{2}\right) \\
& \overrightarrow{\delta_{3}}=a\left(\frac{\sqrt{3}}{2},-\frac{1}{2}\right)
\end{aligned}
$$

There are two atoms in each unit cell of graphene. In graphene only electrons in the $\pi$ bonds formed by $p_{z}$ orbitals contribute to the Dirac properties. The atomic $p_{z}$ orbital wavefunction of each atom can be written as, $\phi\left(\vec{r}-\vec{R}_{j}\right)$. Where $\vec{R}_{j}=$ $m_{j} \overrightarrow{a_{1}}+n_{j} \overrightarrow{a_{2}}$ is an arbitray lattice site, $m$ and $n$ are integers. The normalized trial wavefunction are,

$$
\begin{aligned}
\varphi_{1} & =\frac{1}{\sqrt{N}} \sum_{j} e^{i \vec{k} R_{j}^{A}} \phi\left(\vec{r}-\overrightarrow{R_{j}^{A}}\right) \\
\varphi_{2} & =\frac{1}{\sqrt{N}} \sum_{j} e^{i \vec{k} R_{j}^{B}} \phi\left(\vec{r}-\overrightarrow{R_{j}^{B}}\right)
\end{aligned}
$$

where $N$ is the number of unit cells in the crystal. According to Bloch's theorem, the wavefunction of graphene can be written as a linear combination of the two wavefunctions,

$$
\begin{aligned}
\Psi(\vec{r}) & =c_{1} \varphi_{1}+c_{2} \varphi_{2} \\
& =\frac{1}{\sqrt{N}} \sum_{j}\left[e^{i \vec{k} R_{j}^{A}} c_{1} \phi\left(\vec{r}-\overrightarrow{R_{j}^{A}}\right)+e^{i \vec{k} R_{j}^{B}} c_{2} \phi\left(\vec{r}-\overrightarrow{R_{j}^{B}}\right)\right]
\end{aligned}
$$

These wavefunctions can be substituted in the Schrödinger equation,

$$
H \Psi=E \Psi
$$

which gives,

$$
H\left|c_{1} \varphi_{1}+c_{2} \varphi_{2}\right\rangle=E_{\vec{k}}\left|c_{1} \varphi_{1}+c_{2} \varphi_{2}\right\rangle
$$


Next we multipy with $\left\langle\varphi_{1}\right|$ and $\left\langle\varphi_{2}\right|$.

$$
\begin{aligned}
c_{1}\left\langle\varphi_{1}|H| \varphi_{1}\right\rangle+c_{2}\left\langle\varphi_{1}|H| \varphi_{2}\right\rangle & =E_{k}\left(c_{1}\left\langle\varphi_{1} \mid \varphi_{1}\right\rangle+c_{2}\left\langle\varphi_{1} \mid \varphi_{2}\right\rangle\right) \\
c_{1}\left\langle\varphi_{2}|H| \varphi_{1}\right\rangle+c_{2}\left\langle\varphi_{2}|H| \varphi_{2}\right\rangle & =E_{k}\left(c_{1}\left\langle\varphi_{2} \mid \varphi_{1}\right\rangle+c_{2}\left\langle\varphi_{2} \mid \varphi_{2}\right\rangle\right)
\end{aligned}
$$

In addition, we ignore the overlap of the neighboring $p_{z}$ orbitals, then,

$$
\left\langle\varphi_{i} \mid \varphi_{j}\right\rangle=\delta_{i j}= \begin{cases}1 & \text { if } i=j \\ 0 & \text { if } i \neq j\end{cases}
$$

Rewrite these expressions in a matrix form,

$$
\left|\begin{array}{cc}
\left\langle\varphi_{1}|H| \varphi_{1}\right\rangle-E_{\vec{k}} & \left\langle\varphi_{1}|H| \varphi_{2}\right\rangle \\
\left\langle\varphi_{2}|H| \varphi_{1}\right\rangle & \left\langle\varphi_{2}|H| \varphi_{2}\right\rangle-E_{\vec{k}}
\end{array}\right|=0
$$

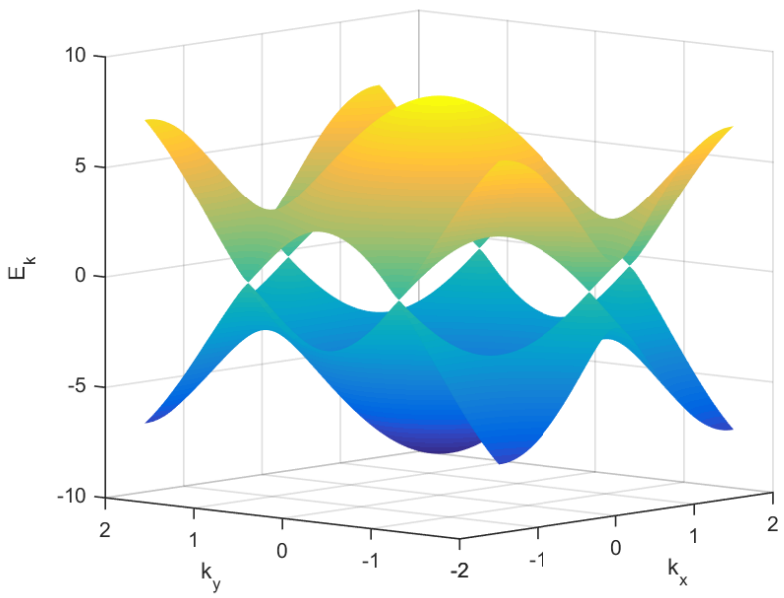

Figure 1.2: Band structure of graphene within the tight-bingding approximation 
The solution is,

$E_{\vec{k}}=\frac{1}{2}\left[\left\langle\varphi_{1}|H| \varphi_{1}\right\rangle+\left\langle\varphi_{2}|H| \varphi_{2}\right\rangle \pm \sqrt{\left(\left\langle\varphi_{1}|H| \varphi_{1}\right\rangle-\left\langle\varphi_{2}|H| \varphi_{2}\right\rangle\right)^{2}+4\left|\left\langle\varphi_{1}|H| \varphi_{2}\right\rangle\right|^{2}}\right]$

If we only consider nearest-neighbors interactions we obtain in the tight-bonding approach,

$$
\begin{aligned}
\left\langle\varphi_{1}|H| \varphi_{2}\right\rangle & =\frac{1}{N} \sum_{j} e^{i \vec{k}\left(R_{j}^{A}-R_{j}^{B}\right)}\left\langle\phi\left(\vec{r}-R_{j}^{A}\right)|H| \phi\left(\vec{r}-R_{j}^{B}\right)\right\rangle \\
& =\left(e^{i \vec{k} \overrightarrow{\delta_{1}}}+e^{i \vec{k} \overrightarrow{\delta_{2}}}+e^{i \vec{k} \overrightarrow{\delta_{3}}}\right)\left\langle\phi\left(\vec{r}-R_{j}^{A}\right)|H| \phi\left(\vec{r}-R_{j}^{B}\right)\right\rangle \\
& =\xi_{\vec{k}} t
\end{aligned}
$$

where,

$$
\begin{aligned}
t & =\left\langle\phi\left(\vec{r}-\overrightarrow{R_{j}^{A}}\right)|H| \phi\left(\vec{r}-\overrightarrow{R_{j}^{B}}\right)\right\rangle \\
\xi_{\vec{k}} & =e^{i \vec{k} \bar{\delta}_{1}}+e^{i \vec{k} \overrightarrow{\delta_{2}}}+e^{i \vec{k} \delta_{3}} \\
& =e^{i k_{y} a}+e^{i\left(-\frac{\sqrt{3}}{2} a k_{x}-\frac{1}{2} k_{y} a\right)}+e^{i\left(\frac{\sqrt{3}}{2} a k_{x}-\frac{1}{2} k_{y} a\right)} \\
& =e^{i a k_{y}}+2 e^{-i a \frac{1}{2} k_{y}} \cos \left(\frac{\sqrt{3}}{2} a k_{x}\right)
\end{aligned}
$$

and,

$$
\begin{aligned}
\left\langle\varphi_{1}|H| \varphi_{1}\right\rangle & =\left\langle\varphi_{2}|H| \varphi_{2}\right\rangle \\
& =\xi_{p}
\end{aligned}
$$

Then,

$$
E_{\vec{k}}=\xi_{p} \pm\left|\xi_{\vec{k}}\right| t
$$


where,

$$
\begin{aligned}
\left|\xi_{\vec{k}}\right| & =\sqrt{\left(e^{i a k_{y}}+2 e^{-i a \frac{1}{2} k_{y}} \cos \left(\frac{\sqrt{3}}{2} a k_{x}\right)\right)\left(e^{-i a k_{y}}+2 e^{i a \frac{1}{2} k_{y}} \cos \left(\frac{\sqrt{3}}{2} a k_{x}\right)\right)} \\
& =\sqrt{3+2 \cos \left(\sqrt{3} k_{y} a\right)+4 \cos \left(\frac{3}{2} k_{x} a\right) \cos \left(\frac{\sqrt{3}}{2} k_{y} a\right)}
\end{aligned}
$$

Set $\xi_{p}=0$,

$$
E_{\vec{k}}= \pm t \sqrt{3+2 \cos \left(\sqrt{3} k_{y} a\right)+4 \cos \left(\frac{3}{2} k_{x} a\right) \cos \left(\frac{\sqrt{3}}{2} k_{y} a\right)}
$$

Based on formula 1.37 we plot the band structure of graphene, see Figure 1.2.

\subsubsection{Germanene}

The unique electronic structure of graphene has inspired the search for other 2D materials with similar properties. Silicon and germanium, like carbon are all group IV elements of the periodic table. Silicene and germanene, the graphene analogues of silicon and germanium respectively, might exhibit similar electronic properties as graphene. Since this thesis deals only with germanene, we will restrict ourselves here to germanene. Germanene is predicted to have a slightly buckled honeycomb lattice (Figure 1.3), which is caused by a weaker $\pi-\pi$ interaction and a distinct coupling of $\sigma$ and $\pi$ bonds. For free-standing germanene, density functional theory (DFT) studies report a buckling in the range of $0.64 \sim 0.74 \AA$, depending on the computational scheme. ${ }^{23}$ However, a small buckling will greatly affect the electronic properties of germanene. The spin-orbit coupling opens a band gap of 23.9 meV in germanene. ${ }^{24}$ This means that germanene can be an ideal candidate to exhibit the quantum spin Hall effect at experimentally accessible temperatures.

In 2005, Kane and Mele 2526 showed that the intrinsic spin-orbit coupling of graphene can open up a gap at the Dirac points. The edge of graphene possess spin-polarized topologically protected edge states. The energy dispersion near Dirac 
points can be written as,

$$
E_{\vec{k}}= \pm \sqrt{\left(v_{F} \hbar \vec{k}\right)^{2}+\Delta_{S O}^{2}}
$$

where $\Delta_{S O}$ is the effective spin-orbit gap. Figure 1.4 shows the schematic diagram of the electronic structure near the Dirac points K and K' without (Figue 1.4(A)) and with (Figue 1.4(B)) spin-orbit coupling. In the cartoon in Figure 1.4(C), the spin up (red line) and spin down (green line) electrons in the edge channels travelling in opposite directions are shown. The intrinsic spin-orbit coupling in graphene is extremely weak, which makes the realization of the quantum spin Hall effect state for graphene almost impossible. For germanene the spin-orbit gap is predicted to be $23.9 \mathrm{meV}$, and therefore germanene is a good candidate to exhibit the quantum spin Hall effect at realistic temperatures. ${ }^{24}$ The first successful realization of the quantum spin Hall effect is reported by the Molenkamp group in University of Würzburg. They used a mercury telluride heterostructure. ${ }^{27}$

\subsection{Scope and outline}

This thesis deals with the synthesis and characterization of metal-induced nanowires on $\mathrm{Ge}(110)$ surface and germanene. The experimental techniques that are used in this thesis are introduced in Chapter 2.

Chapter 3 deals with a study of the bare Ge(110) and Au-induced 1D nanowires by STM and STS. The different reconstructions of Ge(110) surface are explored by
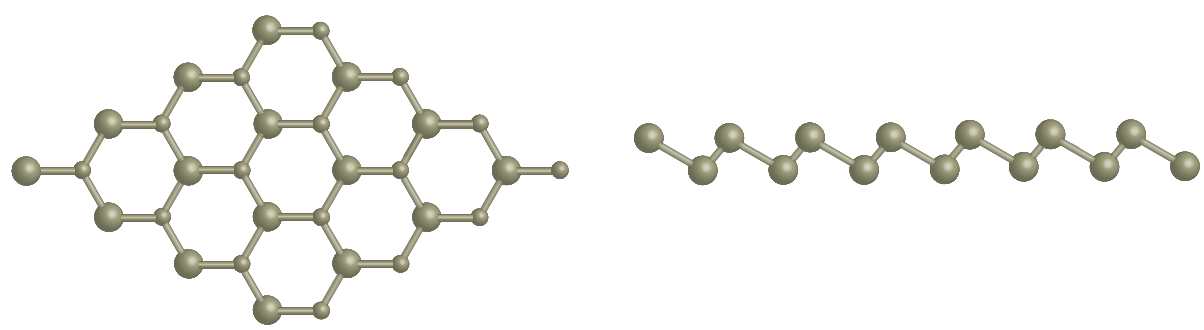

Figure 1.3: Ball and stick model of germanene. The honeycomb lattice is composed of two hexagonal sub-lattices. Left panel: top view. Right panel: side view. 

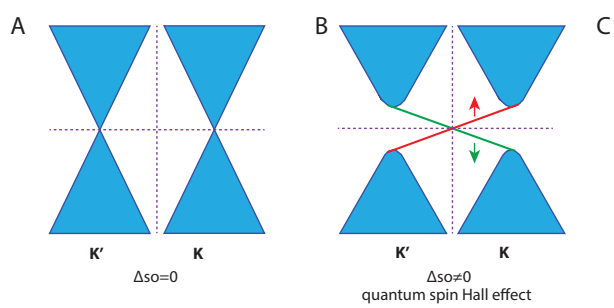

C

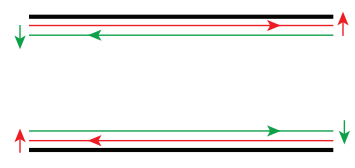

Figure 1.4: Band structure with and without spin orbit coupling

STM. STS data demonstrate three well-defined electronic states. Furthermore, the structural and electronic properties of Au-induced nanowires on $\mathrm{Ge}(110)$ are investigated.

In Chapter 4, the structural and electronic properties of Pt-induced nanowires on $\mathrm{Ge}(110)$ are studied. Various features of nanowires at various Pt coverages are explored by STM and STS.

In Chapter 5, we show that several monolayers of Pt deposited on $\mathrm{Ge}(110)$ followed by annealing at $1100 K$ results in the formation of 3D metallic Pt-Ge nanocrystals. The outermost layer of these crystals exhibits a honeycomb structure. Based on the structural and electronic properties obtained by STM, we propose that the outermost layer of the Pt-Ge nanocrystal is germanene. The zigzag edges of germanene are reconstructed and display a $4 \times$ periodicity. Near the reconstructed germanene zigzag edges the shape of the differential conductivity changes from a V-shape in the interior to a more parabolic-like shape at the edge.

In Chapter 6, we report the successful synthesis of germanene on molybdenum disulfide $\left(\mathrm{MoS}_{2}\right)$, a band gap material. Pre-existing defects in the $\mathrm{MoS}_{2}$ surface act as preferential nucleation sites for the germanene islands. The lattice constant of the germanene layer $(3.8 \pm 0.2 \AA)$ is about $20 \%$ larger than the lattice constant of the $\mathrm{MoS}_{2}$ substrate (3.16 $\mathrm{A}$ ). Scanning tunneling spectroscopy measurements performed on the virtually continuous germanene layers reveal a V-shaped density of states, which is a clear hallmark of a two-dimensional Dirac material. These experimental results are in very good agreement with density functional theory calculations. 


\section{Bibliography}

[1] Feynman, R. P. There's plenty of room at the bottom. Engineering and Science 23, 22-36 (1960).

[2] Luttinger, J. M. An exactly soluble model of a many-fermion system. Journal of Mathematical Physics 4, 1154-1162 (1963).

[3] Haldane, F. D. M. 'Luttinger liquid theory' of one-dimensional quantum fluids. i. properties of the Luttinger model and their extension to the general 1D interacting spinless Fermi gas. Journal of Physics C: Solid State Physics 14, 2585 (1981).

[4] Kane, C. \& Fisher, M. P. Transport in a one-channel Luttinger liquid. Physical Review Letters 68, 1220 (1992).

[5] Yao, Z., Postma, H. W. C., Balents, L. \& Dekker, C. Carbon nanotube intramolecular junctions. Nature 402, 273 (1999).

[6] Bockrath, M. et al. Luttinger-liquid behaviour in carbon nanotubes. Nature 397, 598-601 (1999).

[7] Ishii, H., Kataura, H., Shiozawa, H., Yoshioka, H. et al. Direct observation of Tomonaga-Luttinger-liquid state in carbon nanotubes at low temperatures. Nature 426, 540 (2003).

[8] Lee, J. et al. Real space imaging of one-dimensional standing waves: Direct evidence for a Luttinger liquid. Physical Review Letters 93, 166403 (2004).

[9] Levy, E. et al. Luttinger-liquid behavior in weakly disordered quantum wires. Physical Review Letters 97, 196802 (2006).

[10] Tarucha, S., Honda, T. \& Saku, T. Reduction of quantized conductance at low temperatures observed in 2 to $10 \mu \mathrm{m}$-long quantum wires. Solid State Communications 94, 413-418 (1995).

[11] Auslaender, O. et al. Tunneling spectroscopy of the elementary excitations in a one-dimensional wire. Science 295, 825-828 (2002). 
[12] Park, J. et al. Absence of Luttinger liquid behavior in Au-Ge wires: A highresolution scanning tunneling microscopy and spectroscopy study. Physical Review B 90, 165410 (2014).

[13] de Jong, N. et al. Gold-induced nanowires on the Ge(100) surface yield a 2D and not a 1D electronic structure. Physical Review B 93, 235444 (2016).

[14] Gurlu, O., Adam, O. A., Zandvliet, H. J. W. \& Poelsema, B. Self-organized, one-dimensional Pt nanowires on Ge(001). Applied Physics Letters 83, 46104612 (2003).

[15] Novoselov, K. S. et al. Electric field effect in atomically thin carbon films. Science 306, 666-669 (2004).

[16] van Weperen, I., Plissard, S. R., Bakkers, E. P., Frolov, S. M. \& Kouwenhoven, L. P. Quantized conductance in an InSb nanowire. Nano Letters 13, 387-391 (2012).

[17] Urban, D. F. \& Grabert, H. Interplay of rayleigh and peierls instabilities in metallic nanowires. Physical Review Letters 91, 256803 (2003).

[18] Schäfer, J., Blumenstein, C., Meyer, S., Wisniewski, M. \& Claessen, R. New model system for a one-dimensional electron liquid: self-organized atomic gold chains on Ge(001). Physical Review Letters 101, 236802 (2008).

[19] Deshpande, V. V., Bockrath, M., Glazman, L. I. \& Yacoby, A. Electron liquids and solids in one dimension. Nature 464, 209-216 (2010).

[20] Egger, R., Zazunov, A. \& Yeyati, A. L. Helical Luttinger liquid in topological insulator nanowires. Physical Review Letters 105, 136403 (2010).

[21] Blumenstein, C. et al. Atomically controlled quantum chains hosting a Tomonaga-Luttinger liquid. Nature Physics 7, 776-780 (2011).

[22] Van Hove, L. The occurrence of singularities in the elastic frequency distribution of a crystal. Physical Review 89, 1189 (1953).

[23] Ye, M. et al. Tunable band gap in germanene by surface adsorption. Physica E: Low-dimensional Systems and Nanostructures 59, 60-65 (2014). 
[24] Liu, C.-C., Feng, W. \& Yao, Y. Quantum spin Hall effect in silicene and twodimensional germanium. Physical Review Letters 107, 076802 (2011).

[25] Kane, C. L. \& Mele, E. J. Quantum spin Hall effect in graphene. Physical Review Letters 95, 226801 (2005).

[26] Kane, C. L. \& Mele, E. J. $Z_{2}$ topological order and the quantum spin Hall effect. Physical Review Letters 95, 146802 (2005).

[27] König, M. et al. Quantum spin Hall insulator state in HgTe quantum wells. Science 318, 766-770 (2007). 



\section{Experimental}

The scanning tunneling microscope (STM) was invented in the early 1980s by Gerd Binnig and Heinrich Rohrer who were awarded the Nobel Prize in physics in 1986. STM is an instrument that can be used to image surfaces with atomic resolution. Its resolution is about $0.1 \mathrm{~nm}$ in lateral direction and better than $0.01 \mathrm{~nm}$ in the direction normal to the surface. STM is a fascinating tool for nanoscience and nanotechnology and is widely used to investigate physical, chemical, and biological processes. 


\subsection{The basic principle of STM}

The operation of STM relies on the quantum mechanical tunneling effect. A bias voltage, typically 0.01 to $2 \mathrm{~V}$, is applied between an atomically sharp conductive tip and the sample, that are less than $1 \mathrm{~nm}$ spaced apart from each other. The tunneling current, which is in the range of about 0.01 to $10 \mathrm{nA}$, depends exponentially on the tip-sample distance. For a positive sample bias, electrons tunnel from the tip to the unoccupied states of the sample. Conversely, for a negative sample bias, electrons tunnel from the sample to the empty states of the tip. Using a three-dimensional piezoelectric scanner the tip is scanned across the surface with picometer precision. A schematic view of the STM is shown in Figure 2.1. The tunneling current, in STM, depends strongly on the overlap of the wave functions of tip and surface, which can be described using the well-known Wentzel-Kramer-Brillouin (WKB) approximation: ${ }^{1}$

$$
I \propto \frac{V}{z} \exp (-2 \kappa z)
$$

where $V$ is the applied voltage, $z$ the separation between the tip apex and the substrate and $\kappa$ the decay constant.

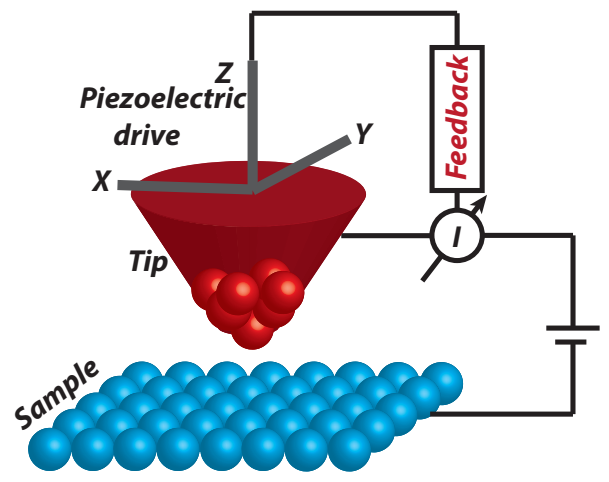

Figure 2.1: Schematic view of the scanning tunneling microscope 
A

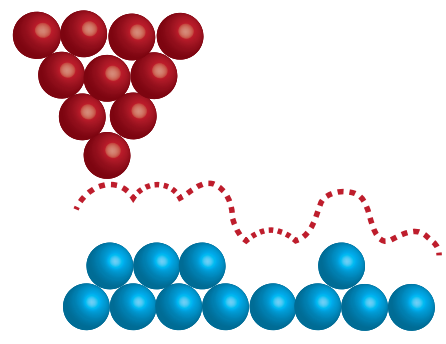

constant current mode
B

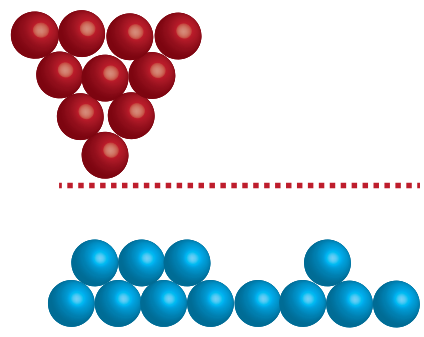

constant hight mode

Figure 2.2: Generic STM operating modes: (A) constant-current and (B) constantheight imaging.

There are two modes of STM operation, the so-called constant current and constant height modes, see Figure 2.2 for a cartoon. In the constant current mode the current is kept constant by adjusting the distance between tip and sample using a feedback loop. An irregular surface can be measured with high precision using the constant current mode, however in order to avoid crashes with the surface, the scan speed of the piezo scanner can not be very high. In the constant height mode, the tip is scanned across the surface while the feedback loop is disabled and therefore the height is kept constant. The constant height mode allows much faster scanning because the system does not have to move the scanner up and down, but it can only be used for relatively smooth surfaces.

\subsubsection{The tunneling effect}

A tunnel junction consists of a metallic tip and a conductive substrate, separated by a small vacuum gap. The simplest model to illustrate the principle of an STM is by considering a one-dimensional potential barrier. A schematic diagram of a onedimensional barrier is depicted in Figure 2.3. $E_{F}$ refers to the Fermi energy, $\Phi_{s}$ and $\Phi_{t}$ to the work functions of the electrodes of sample and tip, respectively, and $d$ is the width of the barrier. Let's consider an electron with an energy of $E$ that encounters a barrier with potential energy $U$. The one-dimensional time-independent 
Schrödinger equation is given by,

$$
-\frac{\hbar^{2}}{2 m} \frac{d^{2} \Psi}{d x^{2}}+U \Psi(x)=E \Psi
$$

where $m$ is the mass of electron, $\Psi(x)$ is the wave function of the electron. When $E>U$, the electron can easily tunnel through the barrier. In case that $E<U$ it is classically forbidden that electron makes it to the other side of the barrier, however in quantum mechanics there is a non-zero probability that the electron can tunnel though the barrier. The solution of the wave function is given by $\Psi(x)=$ $\Psi(0) e^{(-\kappa z)}$, where $\kappa=\frac{\sqrt{2 m(E-U)}}{\hbar}, \kappa$ is referred to as the inverse decay length.

\subsubsection{Tunneling current}

The tunnelling current in STM depends, besides the gap width, also on the electronic structure of the sample and the tip. ${ }^{2}$ In order to reduce the effect of the electronic structure of the tip on the tunneling signal one often selects a metal tip. Most metals have a rather featureless density of states near the Fermi level. Electrochemically etched W wires and mechanically cut PtIr wires are the most commonly used STM

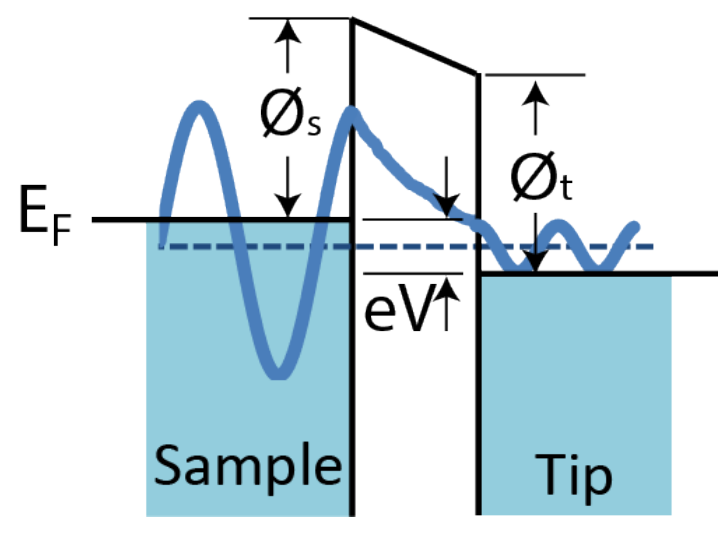

Figure 2.3: Schematic of tunneling between sample and tip under a bias 
tips. Here we briefly address a few models that are used to describe the tunneling process and that go a bit beyond the simplistic one-dimensional barrier model.

Bardeen's approach Bardeen approach ${ }^{3}$ relies on the transfer Hamiltonian method. Using Fermi's golden rule he obtained an expression for the tunneling matrix element for an electron that tunnels from a tip to a substrate, or vice versa, $M_{s t}$ is given by,

$$
M_{s t}=\frac{\hbar^{2}}{2 m} \int\left(\psi_{s}^{*} \vec{\nabla} \psi_{t}-\psi_{t} \vec{\nabla} \psi_{s}^{*}\right) d \vec{S}
$$

where $\psi_{s}^{*}$ and $\psi_{t}$ are the wave function of the two electrodes (sample and tip). In the low bias range and low temperature the tunneling current $I$ in Bardeen's formalism is given by,

$$
I=\frac{4 \pi e}{\hbar} \int_{0}^{e V} \rho_{s}\left(E_{F}-e V+E\right) \rho_{t}\left(E_{F}+e V+E\right)\left|M_{s t}\right|^{2} d E
$$

where $\rho_{s}, \rho_{t}$ are the local density of the states of the sample and the tip, respectively. $V$ is the applied voltage, $M_{s t}$ is the aforementioned tunneling matrix element. From this expression it is immediately obvious that the tunnel current depends on the electronic structure of both electrodes, i.e. tip and substrate. It is also clear that the tunneling current increases with increasing sample bias.

Tersoff-Hamann model Tersoff and Hamann proposed in 1985 a slightly different approach. ${ }^{4}$ They assumed that the apex tip can be modelled by a small sphere with a radius $R$ and subsequently derived constant current contours. Tersoff and Hamann assumed that electronic structure of the tip is featureless and found,

$$
I=32 \pi^{3} \hbar^{-1} e^{2} V \varphi^{2} D_{t}\left(E_{F}\right) R^{2} \kappa^{-4} e^{2 \kappa R} \int \psi_{s}\left(\vec{r}_{0}\right)^{2} d\left(E_{s}-E_{F}\right)
$$

where $\overrightarrow{r_{0}}$ the distance with respect to the center of the tip, $D_{t}$ is the density of the states of the tip; $\psi_{s}$ is the wave function of the sample and $\varphi$ is the work function of the sample. 
Chen's model Chen ${ }^{5,6}$ pointed out that the tunneling current as obtained by Tersoff and Hamann's model is a little too small, which is due to the usage of an $s$-like tip orbital. Chen ${ }^{7}$ proposed a slightly different expression,

$$
\frac{d l n|I|}{d V}=-\frac{e \kappa L}{2 \phi_{s}}+\frac{\rho(e V)|M(e V)|^{2}}{\left|\int_{0}^{e V} \rho_{s}(\epsilon)\right| M(\epsilon)^{2} d \epsilon \mid}
$$

where $L$ is the average distance from the center of the tip atom to the center of the surface atom of the sample, and $\phi_{s}$ is the work function of the sample.

\subsection{Scanning tunneling spectroscopy}

Scanning tunneling spectroscopy (STS) is used to obtain the electronic properties of sample. STS spectra can be recorded simultaneously with a normal STM image. STS allows to study the electronic properties on an atomic scale, which makes it one of the most powerful spectroscopic techniques. Here, we will only mention the three most common STS techniques.

$I-V$ Spectroscopy $I-V$ spectroscopy is current versus bias voltage spectroscopy. During scanning the tip of the STM is kept at a fixed position above the sample with the feedback loop disabled. The potential difference between the sample and the tip (the sample bias) is varied and the tunneling current $I$ is recorded. The density of states, which is proportional to $d I / d V$ for small sample biases, can be obtained by numerical differentiating $I-V$ curves or by adding a small sinusoidal signal to the sample bias and record the $d I / d V$ signal directly with a lock-in amplifier.

$I$ - $t$ Spectroscopy Time-resolved current $(I-t)$ spectroscopy is also a commonly used technique. In this mode to the tip is place at a fixed location with the feedback loop disabled and subsequently the tunneling current is measured as a function of time. The time resolution is determined by the band width of the $I / V$ converter, which is typically in range of $100 \sim 500 \mathrm{kHz}$. Alternatively, the feedback loop can be switched on and the z-piezo voltage can be monitored as a function of time. In the latter mode the time resolution is set by the cut off frequency of the feedback loop. 
$I$ - $Z$ Spectroscopy In current distance $(I-Z)$ spectroscopy the z-piezo is modulated, while the tunneling current is measured. This spectroscopic mode provides information on the local work function or apparent barrier height.

\subsection{Omicron STM-1}

The results presented in this thesis are mainly obtained with a commercially available, Omicron STM-1. The Omicron STM-1 is a room temperature ultra-high vacuum STM. A photograph our UHV system is shown in Figure 2.4. There are three sub-chambers: a preparation chamber, an STM chamber and a load lock chamber. The pressure of the UHV chambers is below $5 \times 10^{-11}$ mbar. The preparation and STM chambers are pumped by a turbo-molecular pump and a ion getter pump integrated with Ti sublimation pump. The load lock is pumped by another turbo-

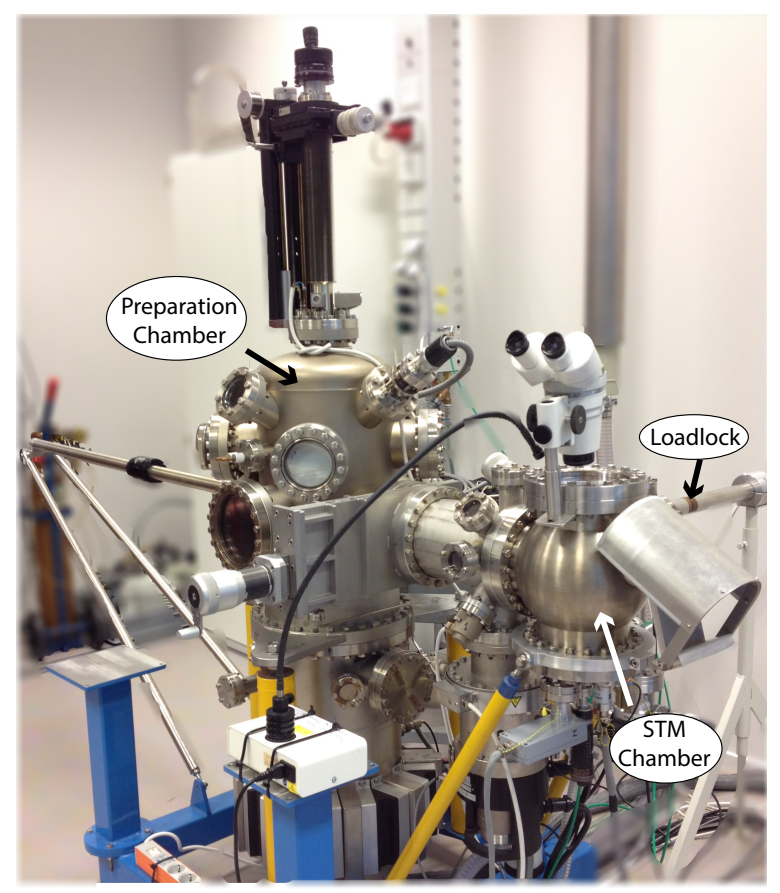

Figure 2.4: Photo of the STM-1 UHV systerm 
molecular pump. The preparation chamber is equipped an ion gun and heating stage for sample cleaning. The system contains several evaporators as well as a quadrupole for rest gas analysis. Samples and tips are stored in a carrousel. 


\section{Bibliography}

[1] Simmons, J. G. Electric tunnel effect between dissimilar electrodes separated by a thin insulating film. Journal of Applied Physics 34, 2581-2590 (1963).

[2] Fischer, Ø., Kugler, M., Maggio-Aprile, I., Berthod, C. \& Renner, C. Scanning tunneling spectroscopy of high-temperature superconductors. Reviews of Modern Physics 79, 353 (2007).

[3] Bardeen, J. Tunnelling from a many-particle point of view. Physical Review Letters 6, 57 (1961).

[4] Tersoff, J. \& Hamann, D. Theory and application for the scanning tunneling microscope. Physical Review Letters 50, 1998 (1983).

[5] Chen, C. J. Tunneling matrix elements in three-dimensional space: The derivative rule and the sum rule. Physical Review B 42, 8841 (1990).

[6] Chen, C. J. Effects of $m \neq 0$ tip states in scanning tunneling microscopy: The explanations of corrugation reversal. Physical Review Letters 69, 1656 (1992).

[7] Chen, C. J. Theory of scanning tunneling spectroscopy. Journal of Vacuum Science \& Technology A 6, 319-322 (1988). 



\section{$\mathrm{Ge}(110)$ surface and Au-induced nanowires}

In this chapter we will study the structural and electronic properties of the bare $\mathrm{Ge}(110)$ surface as well the Au modified Ge(110) surface. The elementary building block of the reconstructed $\mathrm{Ge}(110)$ surface is a pentagon, i.e. a cluster consisting of five germanium atoms. These pentagons can be arranged in different registries. Scanning tunneling microscopy measurements reveal the coexistence of two ordered domains $((16 \times 2)$ and $c(8 \times 10))$ and a disordered domain. Scanning tunneling spectra recorded on the $(16 \times 2), c(8 \times 10)$ and disordered domains are very similar and reveal three well-defined electronic states. Subsequently we have studied the structural and electronic properties of Ge(110) after the deposition of sub-monolayer amounts of $\mathrm{Au}$. Upon Au deposition and annealing nanowires are formed that are aligned along the 'ridges' of the unreconstructed Ge(110) surface (this direction is referred to as the [110] direction). Scanning tunneling spectroscopy measurements recorded on these nanowires reveal that the nanowires are not metallic, suggesting that the nanowires are composed of Ge atoms. 


\subsection{Introduction}

The low-index surfaces of the group IV semiconductors have been studied in great detail, except the (110) surface. ${ }^{1-13}$ This is quite remarkable given the fact that the (110) is intrinsically anisotropic, in contrast to its (100) and (111) counterparts. The surface free energy per unit area of the (110) surface is higher than that of the (100) and (111) surfaces and therefore the (110) surface has the tendency to facet. The bulk truncated Ge(110) surface has a rectangular symmetry and is composed of zigzag rows of atoms that run in the [110] direction. The Ge(110) surface reconstructs into rather large unit cells, which are very complex and involve several atomic layers. The most common surface reconstructions of $\mathrm{Ge}(110)$ are the $(16 \times$ $2)$ and $c(8 \times 10)$ reconstructions. Despite a number of detailed studies there is no full consensus yet on the exact model for these reconstructions. ${ }^{1-11}$ The $(16 \times 2)$ and $c(8 \times 10)$ are both composed of five-membered atom rings, hereafter referred as pentagons and feature small (17 151 ) facets at the steps. The structural models of the $(16 \times 2)$ and $c(8 \times 10)$, as discussed by Ichikawa, ${ }^{8,9}$ are consistent with existing scanning tunneling microscopy data. A careful reflection high energy electron diffraction study by Ichikawa, Fujii and Sugimoto ${ }^{14}$ revealed that prolonged annealing at temperatures below $650 K$ resulted in a $(16 \times 2)$ reconstructed surface. Therefore we can safely conclude that the $(16 \times 2)$ reconstruction is the thermodynamically most stable reconstruction, whereas the $\mathrm{c}(8 \times 10)$ reconstruction is only a metastable and transient reconstruction. The $(16 \times 2)$ reconstruction undergoes an order-disorder transition at a temperature of about $700 \mathrm{~K}$. For a detailed description and discussion of the $(16 \times 2)$ and $\mathrm{c}(8 \times 10)$ reconstructions, as well as a brief overview of the history of the Ge(110) surface we refer to Ichikawa's papers ${ }^{2,3,8-10,14}$ as well as a recent paper by Mullet and Chiang. ${ }^{13}$ The vast majority of papers published on $\mathrm{Ge}(110)$ deal with the structural properties of the surface, whereas the electronic properties received much less attention. ${ }^{5,15}$

Metal-induced self-organizing atomic nanowires on the surface of semiconductors, such as silicon and germanium, as a quasi-1D electron system, have received extensive attention over the past decades. ${ }^{16-23}$ Au atomic chains growing on $\mathrm{Ge}(001)$, initially reported by Wang et al., ${ }^{19}$ revealed the development of $(4 \times 2)$ and $\mathrm{c}(8 \times$ 2) surface reconstructions with metallic properties. The giant missing row (GMR) model, proposed by van Houselt, ${ }^{20}$ is the most accepted model for Au-induced 
nanowires on $\mathrm{Ge}(001) .{ }^{24-26}$ Quite recently a revised GMR model suggested by Seino et al. ${ }^{27}$ is energetically slightly more stable than the previous one. Besides its interesting structural properties, $\mathrm{Au} / \mathrm{Ge}(001)$ systems are considered to the potential candidate for the observation of a Tomonaga-Luttinger liquid (TLL) phenomenon. ${ }^{28,29}$ However, high resolution STM/STS study failed to demonstrate the existence of TLL behavior for this system. ${ }^{25}$ de Jong et al. ${ }^{30}$ combined STM, LEED and ARPES techniques demonstrating this system is a $2 \mathrm{D}$, rather than a $1 \mathrm{D}$ electronic system. As a consequence of the four-fold symmetry of a $\{001\}$ crystal plane, nanowires form $90^{\circ}$ rotated domains, which is contrary to the desire of singledomain nanowires. The (110) surfaces of $\mathrm{Si}$ and $\mathrm{Ge}$, which exhibit in contrast to their (001) and (111) counterparts, anisotropic two-fold in-plane surface symmetry, could be a suitable substrate for the single domain nanowires. Lately, germanium has emerged as a substrate for the wafer-scale growth of graphene. ${ }^{31-33} \mathrm{Ge}(110)$ offers better rotational alignment of graphene domains compared to Ge(111) substrate. ${ }^{34}$ The structural and electronic properties of Au-indued nanowires on Ge(110) have not been studied in great detail, to date only one paper has been published. ${ }^{35}$ Au-induced nanowires on $\mathrm{Si}(110)$ are studied by STM and angle-resolved photoemission showed that the nanowires are metallic. ${ }^{36}$

Here, in this chapter, we present a combined scanning tunneling microscopy and spectroscopy study of the elementary building block, i.e. a pentagon, of the $\mathrm{Ge}(110)$ surface. The local density of states of the two most common reconstructions of the $\mathrm{Ge}(110)$ surface, i.e. the $(16 \times 2)$ and $\mathrm{c}(8 \times 10)$ reconstructions, will be extracted from spatially resolved scanning tunneling spectra. The formation of nanowires after deposition of Au on Ge(110) are also studied by STM/STS and low energy electron microscopy (LEEM).

\subsection{Experimental details}

Experiments were performed with an Omicron room temperature scanning tunneling microscope (STM-1) operating in an ultra-high vacuum (UHV) chamber with a base pressure below $5 \times 10^{-11}$ mbar. The Ge(110) samples were cut from nominally flat, single-side-polished n-type substrates. Samples were mounted on Mo holders and the contact to any other metal during preparation and experiment was carefully avoided. The Ge(110) samples have been degassed for about 24 hours at 
$700 \mathrm{~K}$ and subsequently cleaned by the method described in detail in ref. ${ }^{37}$ This cleaning method involves several cycles of Argon ion bombardment followed by annealing at temperatures of $1100( \pm 25) K$. After five to seven of these cleaning cycles the $\mathrm{Ge}(110)$ samples were atomically clean and exhibited well-ordered reconstructed $(16 \times 2)$ and $\mathrm{c}(8 \times 10)$ domains as well as some disordered regions. The relative occupation of the various reconstructions can be tuned by varying the cooling time after a high temperature anneal. A slow cooling rate leads to an increase of the $(16 \times 2)$ domains at the expense of the $c(8 \times 10)$ and disordered domains. Since we aim at a detailed study of all domains we have rapidly cooled down our samples.

Subsequently, Au is deposited on $\mathrm{Ge}(110)$ subtrate by resistively heating a W wire wrapped with high purity $\mathrm{Au}(99.995 \%)$. Ge(110) substrate is kept at room temperature. After Au deposition, the sample was annealed at $750( \pm 25) K$ for 5 minutes and subsequently cooled down to room temperature before observation in the STM. Low energy electron diffraction was also employed in these experiments.

All the STM observations are obtained in constant-current mode with electrochemical etched tungsten tips.

\subsection{Properties of the bare $\mathrm{Ge}(110)$ surface}

In Figure 3.1(A) a large-scale filled-state STM image of a Ge(110) surface is shown. The Ge $(110)$ surface exhibits regions with $c(8 \times 10)$ and $(16 \times 2)$ reconstructions, as well as some disordered regions. In the insets of Figure 3.1 zoom-ins of the different phases are displayed. Interestingly, all phases, including the disordered phase, are composed of five-membered atom rings (hereafter referred to as pentagons). In Figures 3.2(A) and 3.2(B) simplified structural models of the $(16 \times 2)$ and $\mathrm{c}(8 \times$ 10) reconstructions are shown. For the fully relaxed models we refer to the work of Ichikawa. ${ }^{8,9}$ In these papers fully relaxed models of the $(16 \times 2)$ and the $c(8 \times 10)$ reconstructions are shown that are consistent with existing STM data. The zigzag

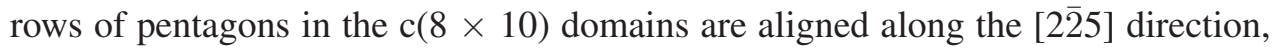
whilst the zigzag rows of pentagons in the $(16 \times 2)$ domains are aligned along the [11̄2] direction. Both straight and rough step edges are found on the surface. The straight steps are aligned along the zigzag pentagon rows of the $(16 \times 2)$ domains, i.e. along the [11 2$]$ direction. Both zigzag rows seem to be composed of pentagon 
twins, however a more careful inspection of the models (see refs. ${ }^{8,9}$ ) reveals that the pentagon twins are not exactly the same. There is another new reconstruction also found by the same sample preparation procedure. In Figure 3.3(A), a large-scale scanning tunneling microscopy image of the bare Ge(110) surface is shown. Figure $3.3(\mathrm{~B})$ is the zoom in image on the $(16 \times 2)$ reconstruction of Figure 3.3(A). Figure 3.3(B), shows a new reconstruction, which looks similar to the $(16 \times 2)$ reconstruction but is in fact a bit different. There is a 30 degree angle between the $(16 \times 2)$ pentagon twins and the twins of this new reconstruction. Furthermore, there is a dimer in between every pentagon twin.

In Figure 3.4(A) the $I-V$ spectrum of the bare Ge(110) surface is depicted. The $I-V$ curves recorded on the different regions of the $\mathrm{Ge}(110)$ surface, i.e. the $(16 \times 2)$, $c(8$ $\times 10)$ and disordered domains, are essential the same. This is not so strange since all reconstructions/phases are composed of the same elementary building blocks, i.e.

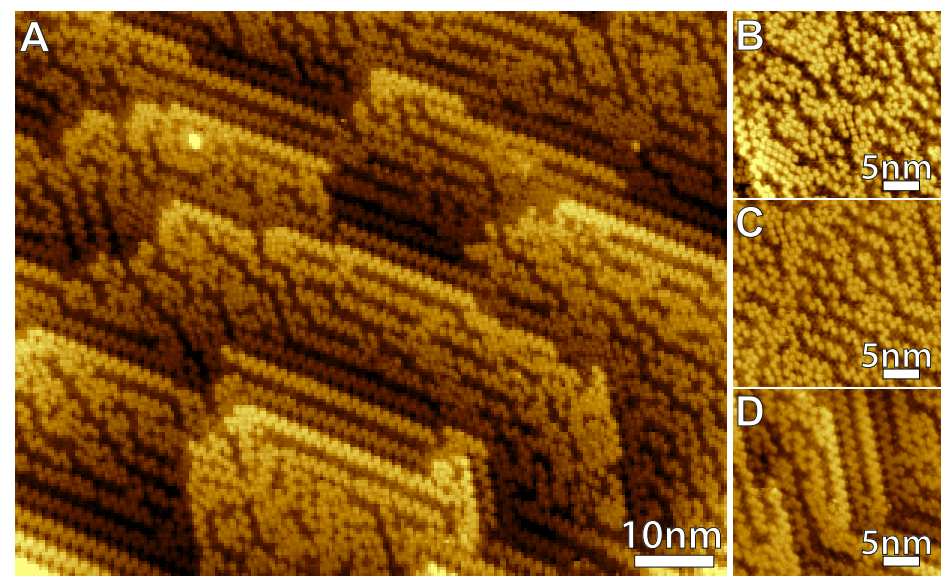

Figure 3.1: (A) Filled-state scanning tunneling microscopy image of Ge(110).

(B) Scanning tunneling microscope image of the disordered phase.

(C) Scanning tunneling microscope image showing a region that exhibits a $c(8 \times 10)$ phase (middle part of the image) as well as a disordered phase (at the left and right border of the image).

(D) Scanning tunneling microscope image of the $(16 \times 2)$ phase.

Set points are in (A) $-1.5 \mathrm{~V}, 0.5 \mathrm{nA},(\mathrm{B})-1.5 \mathrm{~V}, 0.29 \mathrm{nA},(\mathrm{C})-1.5 \mathrm{~V}, 0.29 \mathrm{nA}$ and (D) $-1.5 \mathrm{~V}$ and $0.29 \mathrm{nA}$. 


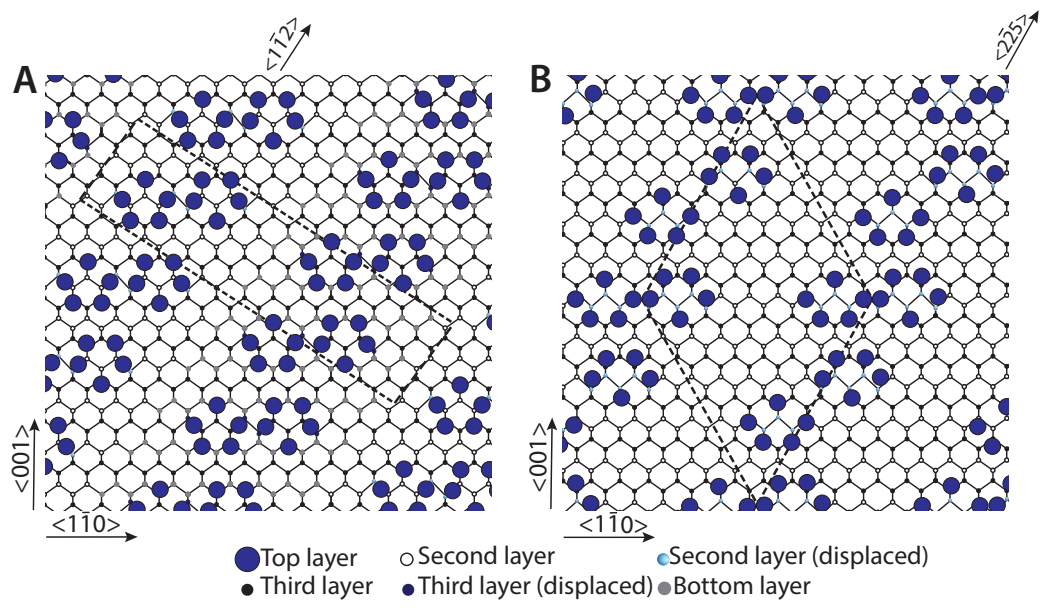

Figure 3.2: Simplified ball-and-stick models of the $(16 \times 2)$ reconstruction $(A)$ and the $\mathrm{c}(8 \times 10)$ reconstruction $(\mathrm{B})$. Fully relaxed models for both reconstructions can be found in refs. ${ }^{8,9}$
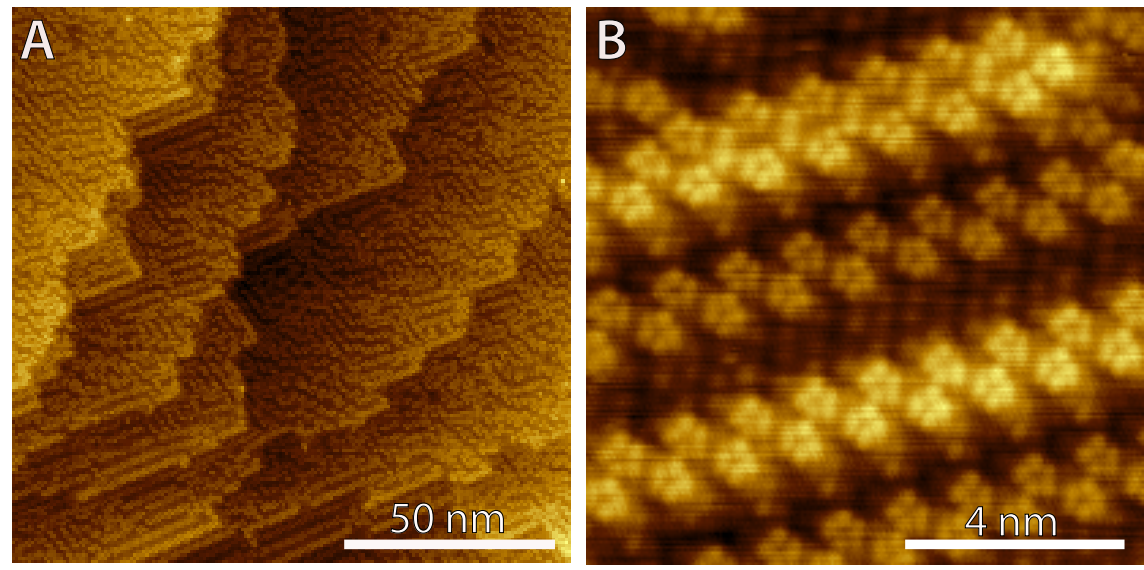

Figure 3.3: Scanning tunneling microscopy image of the bare surface of $\mathrm{Ge}(110)$, (A) large-scale STM image (B). Small scale, high resolution STM image (A). The setpoints are A: $-1.03 \mathrm{~V} 1.01 \mathrm{nA} \mathrm{B}:-1.03 \mathrm{~V}, 0.50 \mathrm{nA}$ 
the pentagons. In Figure 3.4(B) and 3.4(C) the differential conductivity $(d I / d V)$ and the normalized differential conductivity $((d I / d V) /(I / V))$ are shown. All $I-V$ traces are recorded with the same set points of $\mathrm{V}=-1.5 \mathrm{~V}$ and $\mathrm{I}=0.25 \mathrm{nA}$. In Figure 3.4(D) an STM image recorded at the used set points is depicted. In the normalized differential conductivity spectrum three well-defined peaks are resolved: two filledstates at $-1.1 \mathrm{~V}$ and $-0.3 \mathrm{~V}$ respectively and an empty-state at $0.4 \mathrm{~V}$. In Figures 3.4(E) and 3.4(F) STM images at the peak positions $0.4 \mathrm{~V}$ and $-0.35 \mathrm{~V}$ are shown. In contrast to Figure 3.4(D), where a clear structure in between the zigzag pentagon

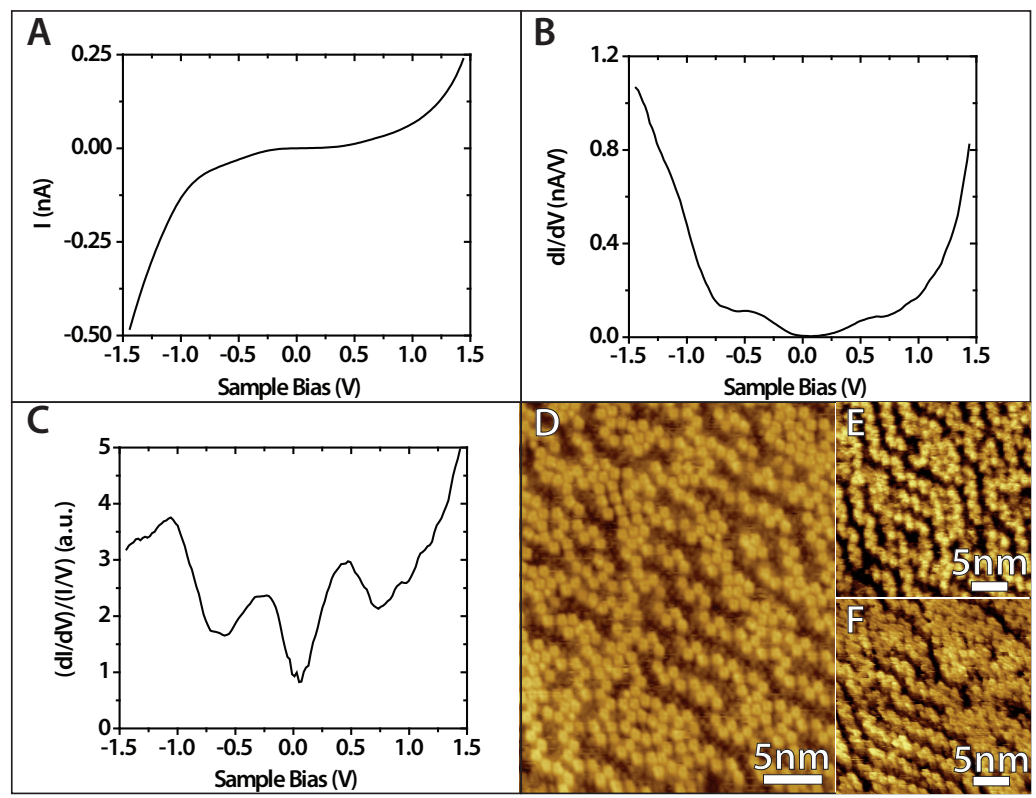

Figure 3.4: (A) Current-voltage (I-V) spectrum of the Ge(110) surface.

(B) Differential conductivity $(d I / d V)$ versus sample bias of the $\mathrm{Ge}(110)$ surface.

(C) Normalized differential conductivity $(d I / d V) /(I / V)$ versus sample of the $\mathrm{Ge}(110)$ surface. Set points for (A), (B) and (C) are $-1.5 \mathrm{~V}, 0.25 \mathrm{nA}$.

(D) Scanning tunneling microscope image of the $\mathrm{Ge}(110)$ surface at $-1.5 \mathrm{~V}$ and 0.29 nA.

(E) Scanning tunneling microscope image of the $\mathrm{Ge}(110)$ surface taken at $0.4 \mathrm{~V}$ and $0.15 \mathrm{nA}$.

(F) Scanning tunneling microscope image of the Ge(110) surface taken at $-0.35 \mathrm{~V}$ and $0.15 \mathrm{nA}$ 
rows appears, Figures 3.4(E) and 3.4(F) are composed of pentagons only.

Since to the best of our knowledge no spectroscopic data of the Ge(110) is available, we compare our results with spectroscopic data recorded on the closely related $\mathrm{Si}(110)-(16 \times 2)$ surface. ${ }^{38}$ Setvínet al. ${ }^{38}$ performed a very detailed study on the electronic structure of the $\mathrm{Si}(110)$ surface. In contrast to the Ge(110) surface, Setvín et al. found at least four electronic states for the $\operatorname{Si}(110)$ surface. Two electronic states are located very close to the Fermi level, one about $0.2 \mathrm{eV}$ below the Fermi level and one about $0.2 \mathrm{eV}$ above the Fermi level. These two states near the Fermi level can be ascribed to the pentagons, which are also the elementary building blocks of the $\mathrm{Si}(110)-(16 \times 2)$ surface. Another filled state at $-1.5 \mathrm{~V}$ is located in the middle of the pentagons and was ascribed to the underlying zigzag line of $\mathrm{Si}$ atoms. The energy of this electronic state is high enough to be assigned to $\mathrm{Si}-\mathrm{Si}$ back bonds. The electronic states we have found for $\mathrm{Ge}(110)$ at $-0.3 \mathrm{~V}$ and $0.4 \mathrm{~V}$ are both related to the pentagons, but our room temperature STS data has insufficient spatial resolution to resolve the exact position of the electronic states within the pentagons. The electronic state of $\mathrm{Ge}(110)$ located at $-1.1 \mathrm{~V}$ is also clearly present in the troughs between the zigzag pentagon rows and therefore we tentatively ascribe this state to Ge-Ge back bonds. For $\mathrm{Si}(110)-(16 \times 2)$ two more empty states are reported, ${ }^{38}$ one located at $1.2 \mathrm{~V}$ and another located at about $1.6 \sim 2.0 \mathrm{eV}$ above the Fermi level. Since we have not found any empty states up to $1.5 \mathrm{eV}$ for $\mathrm{Ge}(110)$ we will not further elaborate on these empty states.

\subsection{Au-induced nanowires}

After the deposition of sub-monolayer amont of $\mathrm{Au}$ on the $\mathrm{Ge}(110)$ and annealing at $750( \pm 25) K$ for 5 minutes self-organized atomic nanowires are formed. In Figure 3.5(A), a large scale filled-state STM image is shown. The nanowires have a well-defined width and extend to several tens of nanometers long, occasionally interrupted by defects or phase shifts. In contrasts to the $\mathrm{Ge}(001) / \mathrm{Au}$ system, the Au-induced self-organized nanowires on the $\mathrm{Ge}(110)$ substrate are aligned in the same crystallographic direction, namely the [1 $1 \overline{1} 0]$ direction. Figure 3.5(B) is a high resolution, zoom-in version of Figure 3.5(A). The most prominent structures in Figure 3.5(B) are the bright zigzag chains, which hereafter are referred to as nanowires. The atomic rows of the bare $\mathrm{Ge}(110)$ terrace also run along the [110] direction and 
are often comprised of bright and dim spots. There is no order in the arrangement of these bright and dim spots (highlighted by the light-blue ellipse). The bright zigzag nanowires mainly consist of multi-segmented features highlighted by the wiggled black lines in Figure 3.5(B). The double rows of protrusions can be in-phase or outof-phase. The height of the nanowires is around $2.5 \AA \quad$ (see the line profile shown in Figure 3.5(F)). In our study of the $\mathrm{Pt} / \mathrm{Ge}(110)$ system, the pentagons, characteristic of the bare $\mathrm{Ge}(110)$ surface, were partly preserved at low nanowire coverages. However, for the $\mathrm{Au} / \mathrm{Ge}(110)$ system we did not even find a single pentagon, indicating that the Au leads to a severe restructuring of the Ge(110) surface. It is
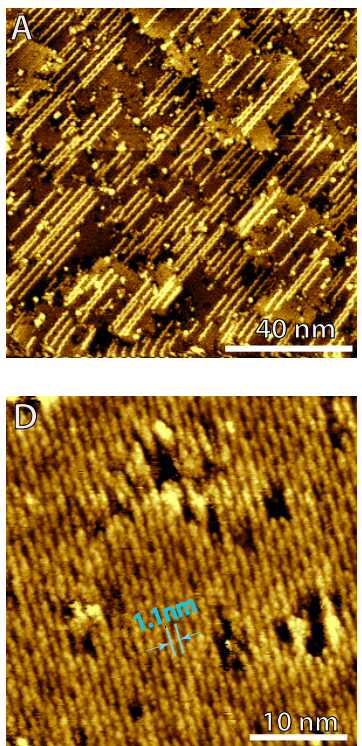
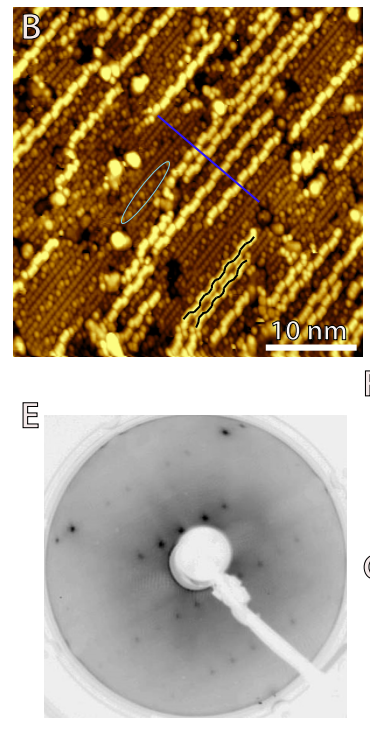
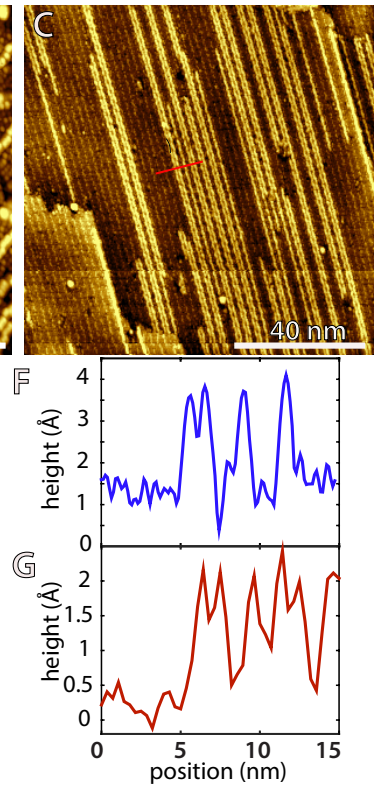

Figure 3.5: (A) large-scale STM image of Ge(110) after the deposition of a submonolayer amount of Au.

(B) small-scale STM image of Ge(110) after a sub-monolayer amount of Au.

(C) and (D) STM images of Ge(110) after the deposion of 1 monolayer of Au.

(E) LEED pattern, acquired at an electron energy of $120 \mathrm{eV}$.

(F) line profile in $(B),(G)$ line profile in $(C)$.

The set points are (A) $-1.67 \mathrm{~V}, 0.5 \mathrm{nA},(\mathrm{B})-1.85 \mathrm{~V}, 0.5 \mathrm{nA}$; (C) $-1.5 \mathrm{~V}, 0.6 \mathrm{nA}$, (D) $-1.23 \mathrm{~V}, 0.6 \mathrm{nA}$ 
important to point out here that the distance between the substrate rows is $5.65 \AA$ and the separation between the features within these substrate rows is $4.0 \AA$. These values correspond to, $a_{0}$, the lattice constant of germanium, and $\frac{1}{2} \sqrt{2} a_{0}$, respectively. This is a clear indication that the Ge(110) completely de-reconstructs upon the deposition of Au. By increasing the amount of Au the density of nanowires increases, see Figure 3.5(C). For Au coverages that exceed one monolayer a new type of nanowire emerges, see Figure 3.5(D). The separation between these slightly wiggling nanowires is $2 a_{0}$, i.e. $1.1 \mathrm{~nm}$, whereas the periodicity within the nanowires is $0.4 \mathrm{~nm}$. In Figure 3.5(E) a low energy electron diffraction (LEED) pattern of a $\mathrm{Ge}(110)$ surface after the deposition of about one monolayer of Au is shown. The $(2 \times 1)$ periodicity nicely agrees with the STM observations. The line profile shown in Figure 3(F) reveals that the trenches between the nanowires have a depth of about $2.5 \AA$ A. Recently, Watanabe et al. ${ }^{35}$ studied the Ge(110)/Au system using scanning tunneling microscopy. Their STM images qualitatively agree with our observations, however the distance between the nanowires, that they extracted from their STM images are at variance with our observations.

In order to study the electronic properties of the Au-induced atomic nanowires, we recorded local tunneling spectroscopy measurements on the nanowires as well as on the nanowire-free regions (see inset Figure 3.6). The differential conductance, obtained by numerically differentiating of the current-voltage traces ( $I-V$ spectra), are shown in Figure 3.6. The red and black lines refer to the spectra recorded on the nanowires and the nanowire-free regions, respectively. The differential conductivity curves of both regions reveal a band gap region near the Fermi level indicating that both regions are semiconducting. Based on these observations we tentatively conclude that the deposited $\mathrm{Au}$ atoms have diffused to sub-surface positions and therefore we arrive at the conclusion that the Au-induced nanowires are composed of Ge atoms.

\subsection{Conclusions}

In summary, we have used scanning tunneling microscopy and spectroscopy to study the structural and electronic properties of the Ge(110) surface. We observed the coexistence of three different phases at room temperature: the $(16 \times 2)$ phase, the $\mathrm{c}(8 \times 10)$ phase and a disordered phase. The elementary building block of 


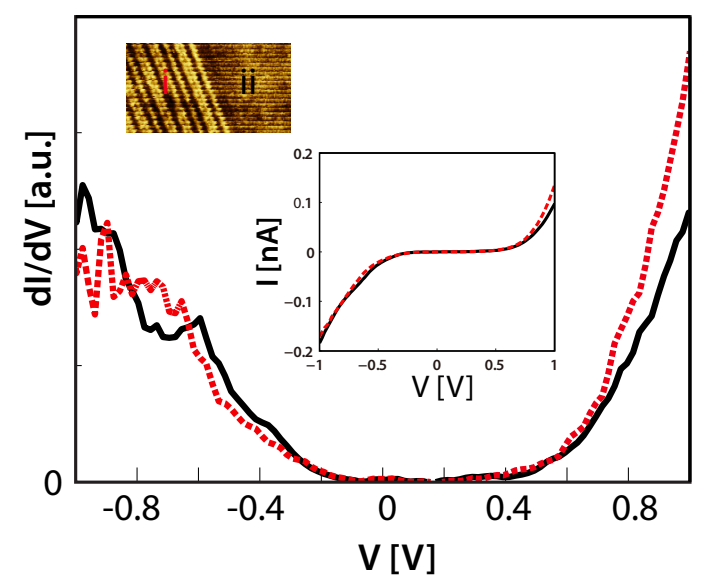

Figure 3.6: $d I / d V$ on the wires and off the wires, respectively. The set point for the IV spectra are $-1.5 \mathrm{~V}$ and $0.6 \mathrm{nA}$

three phases is a five-membered atom ring (pentagon). Scanning tunneling spectra recorded at the different phases reveal that there are hardly any differences between the phases. The scanning tunneling spectra exhibit three well-defined electronic states: two filled-states located $1.1 \mathrm{eV}$ and $0.3 \mathrm{eV}$ below the Fermi level and an empty state which is positioned $0.4 \mathrm{eV}$ above the Fermi level. The electronic states at $-0.3 \mathrm{eV}$ and $0.4 \mathrm{eV}$ can be ascribed to the pentagons, whilst we tentatively ascribe the electronic state located at $-1.1 \mathrm{eV}$ to Ge-Ge back bonds.

We have studied the structural and electronic properties of Au-induced atomic nanowires on $\mathrm{Ge}(110)$ by scanning tunneling microscopy, scanning tunneling spectroscopy and low energy electron microscopy. There are two types of atomic nanowires that both run along the [110] direction. Scanning tunneling spectroscopy measurements reveal that the $\mathrm{Au}$-induced atomic nanowires are semiconducting, suggesting that the nanowires are compose of germanium atoms. 


\section{Bibliography}

[1] Olshanetsky, B. Z., Repinsky, S. M. \& Shklyaev, A. A. LEED investigation of germanium surfaces cleaned by sublimation of sulphide films: structural transitions on clean Ge(110) surface. Surface Science 64, 224-236 (1977).

[2] Noro, H. \& Ichikawa, T. RHEED study of surface reconstruction at clean Ge(110) surface. Japanese Journal of Applied Physics 24, 1288 (1985).

[3] Ichikawa, T. et al. Ge(110) surface reconstruction observed with scanning tunneling microscopy. Solid State Communications 93, 541-545 (1995).

[4] Gai, Z., Zhao, R. G. \& Yang, W. S. Atomic structure of the Ge(101) surface. Physical Review B 57, R6795 (1998).

[5] Santoni, A., Petaccia, L., Dhanak, V. \& Modesti, S. High-temperature phase transitions at the Ge(110) surface. Surface Science 444, 156-162 (2000).

[6] Kim, H., Vailionis, A., Cahill, D. G. \& Greene, J. E. Ge(011)-c $(8 \times 10)$ surface structure and hydrogen desorption pathways: a temperature-programmed desorption and scanning tunneling microscopy study. Surface Science 457, 337-344 (2000).

[7] Takeuchi, N. Bond conserving rotation, adatoms and rest atoms in the reconstruction of $\mathrm{Si}(110)$ and $\mathrm{Ge}(110)$ surfaces: a first principles study. Surface Science 494, 21-27 (2001).

[8] Ichikawa, T. An ab initio study on the atomic geometry of reconstructed Ge(110) $16 \times 2$ surface. Surface Science 544, 58-66 (2003).

[9] Ichikawa, T. Atomic geometry of the $\mathrm{Ge}(110) \mathrm{c}(8 \times 10)$ structure. Surface Science 560, 205-212 (2004).

[10] Ichikawa, T. In situ stm observations of ordering behaviors on Ge(110) surfaces and atomic geometry of the $\mathrm{Ge}\{17151\}$ facet. Surface Science 560, 213-225 (2004).

[11] Stekolnikov, A. A., Furthmüller, J. \& Bechstedt, F. Structural elements on reconstructed Si and Ge(110) surfaces. Physical Review B 70, 045305 (2004). 
[12] Ohira, Y., Yoshimura, M. \& Ueda, K. Metastable phase of Si(110) surface: $5 \times 8$ reconstruction. Japanese Journal of Applied Physics 46, 5652 (2007).

[13] Mullet, C. H. \& Chiang, S. Reconstructions and phase transition of clean Ge(110). Surface Science 621, 184-190 (2014).

[14] Ichikawa, T., Fujii, H. \& Sugimoto, A. STM and RHEED study of Ge(110) reconstructions. SPRINGER PROCEEDINGS IN PHYSICS 87, 297-298 (2001).

[15] Popik, T. Y., Feyer, V., Shpenik, O. \& Popik, Y. V. The specific features of lowenergy electron backscattering from different Ge surfaces. Surface Science 499, L113-L118 (2002).

[16] Nogami, J., Park, S.-i. \& Quate, C. F. Indium-induced reconstructions of the Si(111) surface studied by scanning tunneling microscopy. Physical Review B 36, 6221 (1987).

[17] Oncel, N. et al. Quantum confinement between self-organized Pt nanowires on Ge(001). Physical Review Letters 95, 116801 (2005).

[18] Gurlu, O., Adam, O. A., Zandvliet, H. J. W. \& Poelsema, B. Self-organized, one-dimensional Pt nanowires on Ge(001). Applied Physics Letters 83, 4610 4612 (2003).

[19] Wang, J., Li, M. \& Altman, E. I. Scanning tunneling microscopy study of selforganized Au atomic chain growth on ge(001). Physical Review B 70, 233312 (2004).

[20] van Houselt, A., Fischer, M., Poelsema, B. \& Zandvliet, H. J. W. Giant missing row reconstruction of $\mathrm{Au}$ on $\mathrm{Ge}(001)$. Physical Review B 78, 233410 (2008).

[21] Stekolnikov, A. A., Bechstedt, F., Wisniewski, M., Schäfer, J. \& Claessen, R. Atomic nanowires on the $\mathrm{Pt} / \mathrm{Ge}(001)$ surface: Buried Pt-Ge versus top Pt-Pt chains. Physical Review Letters 100, 196101 (2008).

[22] Ma, X.-D. et al. Strain relief guided growth of atomic nanowires in a $\mathrm{Cu}_{3} \mathrm{~N}$ $\mathrm{Cu}(110)$ molecular network. Physical Review Letters 102, 205503 (2009). 
[23] Oncel, N. Atomic chains on surfaces. Journal of Physics: Condensed Matter 20, 393001 (2008).

[24] Niikura, R., Nakatsuji, K. \& Komori, F. Local atomic and electronic structure of Au-adsorbed $\mathrm{Ge}(001)$ surfaces: Scanning tunneling microscopy and x-ray photoemission spectroscopy. Physical Review B 83, 035311 (2011).

[25] Park, J. et al. Absence of luttinger liquid behavior in Au-Ge wires: A highresolution scanning tunneling microscopy and spectroscopy study. Physical Review B 90, 165410 (2014).

[26] Lichtenstein, T., Teiken, H., Pfnür, H., Wollschläger, J. \& Tegenkamp, C. Auchains grown on Ge (100): A detailed SPA-LEED study. Surface Science 632, 64-70 (2015).

[27] Seino, K. \& Bechstedt, F. Atomic configurations of Au-induced nanowires on Ge(001) stabilized by higher Au coverages. Physical Review B 93, 125406 (2016).

[28] Blumenstein, C. et al. Atomically controlled quantum chains hosting a Tomonaga-Luttinger liquid. Nature Physics 7, 776-780 (2011).

[29] Heimbuch, R., Kuzmin, M. \& Zandvliet, H. J. W. Origin of the Au/Ge (001) metallic state. Nature Physics 8, 697 (2012).

[30] de Jong, N. et al. Gold-induced nanowires on the Ge(100) surface yield a 2D and not a 1D electronic structure. Physical Review B 93, 235444 (2016).

[31] Wang, G. et al. Direct growth of graphene film on germanium substrate. Scientific Reports 3 (2013).

[32] Lee, J.-H. et al. Wafer-scale growth of single-crystal monolayer graphene on reusable hydrogen-terminated germanium. Science 344, 286-289 (2014).

[33] Lippert, G. et al. Graphene grown on ge (001) from atomic source. Carbon 75, 104-112 (2014).

[34] Rogge, P. et al. On the rotational alignment of graphene domains grown on $\mathrm{Ge}(110)$ and $\mathrm{Ge}(111)$. MRS Communications 5, 539-546 (2015). 
[35] Watanabe, T., Yamada, Y., Sasaki, M., Sakai, S. \& Yamauchi, Y. Pt-and Auinduced monodirectional nanowires on Ge(110). Surface Science 653, 71-75 (2016).

[36] McChesney, J., Crain, J., Himpsel, F. \& Bennewitz, R. Si(110)-5 × 2- Au: A metallic chain structure. Physical Review B 72, 035446 (2005).

[37] Zandvliet, H. J. W. The Ge(001) surface. Physics Reports 388, 1-40 (2003).

[38] Setvín, M. et al. Electronic structure of $\operatorname{Si}(110)-(16 \times 2)$ studied by scanning tunneling spectroscopy and density functional theory. Physical Review B 84, 115317 (2011). 



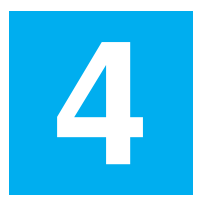

\section{Pt-induced nanowires on $\mathrm{Ge}(110)$}

In this chapter the structural and electronic properties of Pt-induced nanowires on Ge(110) surface are studied by scanning tunneling microscopy and low energy electron microscopy. The deposition of a sub-monolayer amount of Pt and subsequent annealing at $1100( \pm 25) \mathrm{K}$ results into nanowires which are aligned along the densely packed [110] direction of the Ge(110) surface. With increasing Pt coverage the nanowires form densely packed arrays with separations of $1.1 \pm 0.1 \mathrm{~nm}, 2.0 \pm$ $0.1 \mathrm{~nm}$ and $3.4 \pm 0.1 \mathrm{~nm}$. Ge pentagons reside in the troughs for nanowire separations of $3.4 \pm 0.1 \mathrm{~nm}$, however for smaller nanowire separations no pentagons are found. Spatially resolved scanning tunneling spectroscopy measurements reveal a filled electronic state at $-0.35 \mathrm{eV}$. This electronic state is present in the troughs as well as on the nanowires. The $-0.35 \mathrm{eV}$ state has the strongest intensity on the pentagons, which is in line with the results of the bare Ge(110) surface in the previous chapter. For Pt depositions exceeding two monolayers, pentagon free nanowire patches are found, that coexist with Ge-Pt clusters. Upon annealing at $1040 \mathrm{~K}$ these Ge-Pt clusters become liquid-like, indicating that we are dealing with eutectic $P t_{0.22} \mathrm{Ge}_{0.78}$ clusters. Low energy electron microscopy videos reveal the formation and spinodal decomposition of these eutectic Ge-Pt clusters. 


\subsection{Introduction}

In the past two decades the growth of self-assembled nanowires, with widths of only a few atoms and lengths up to micrometers, have attracted a lot of attention. ${ }^{1-13}$ This interest is due to the technological importance of nanowires, for instance as elementary electronic devices or as the ultimate nanoscale interconnections between electronic components on integrated circuits. But also from a scientific point of view nanowires, which can often be considered as quasi one-dimensional electron systems, are very interesting. Examples of intriguing and interesting physical properties of these quasi one-dimensional systems are spin and charge density waves ${ }^{14,15}$ and spin charge separation. ${ }^{16-18}$

The (110) surfaces of $\mathrm{Si}$ and Ge are, in contrast to their (001) and (111) counterparts, intrinsically anisotropic, which can be very beneficial for some practical applications. In addition the (110) surface free energy is substantially higher than the surface free energies of the (001) and (111) surfaces. The Ge(110) surface exhibits a rather complicated surface reconstruction. ${ }^{19,20}$ Despite the considerable attention for the $5 \mathrm{~d}$ transition metal ( $\mathrm{Au}, \mathrm{Pt}$, and Ir) induced nanowires on $\mathrm{Ge}(001),{ }^{2,4-6,21}$ not much attention has been yet paid to the deposition of the these $5 \mathrm{~d}$ transition metals on $\mathrm{Ge}(110)$.

However, during the last decade several reports on the deposition of Dy, Co, Ag, Au, $\mathrm{Pt}, \mathrm{Gd}, \mathrm{Y}, \mathrm{Mn}, \mathrm{Fe}$ and Ir on the closely related $\mathrm{Si}(110)$ surface have appeared. ${ }^{22-32}$ Visikovskiy and co-authors studied the $\mathrm{Pt} / \mathrm{Si}(110)$ system and they found four different nanowire phases for coverages smaller than one monolayer (1 ML), ${ }^{26}$ while for more than $1 \mathrm{ML}$ Pt they reported the formation of 3D silicides. ${ }^{27}$ Recently, Park et $a l .{ }^{29}$ studied the formation of Pt induced nanowires on Si(110) after the deposition of 1 ML Pt using angle-resolved photo-emission spectroscopy (ARPES) and scanning tunneling microscopy and spectroscopy (STM/STS). ${ }^{29}$ They found regularly spaced NWs aligned along the [1 $1 \overline{1} 0]$ direction with a periodicity along the NWs of 3 times the unit cell of the underlying $\operatorname{Si}(110)$ surface. Along the NWs they found a one-dimensional electronic channel, with characteristic features of a Rashba-type band splitting in the density of states and band dispersions. ${ }^{29}$

Compared to $\mathrm{Si}, \mathrm{Ge}$ has a smaller band gap, higher hole mobility and hole concentration, which is advantageous for high-speed devices, for instance p-channel transistors. ${ }^{33}$ Furthermore, Ge can achieve higher switching speeds than $\mathrm{Si}$, which 
could enable the development of faster chips. Metal induced nanowires on $\mathrm{Ge}(110)$ are therefore technologically and fundamentally interesting. The surface free energy per unit area of the (110) surface is higher than that of the (100) and (111) surfaces and therefore the (110) surface has the tendency to facet. The bulk truncated $\mathrm{Ge}(110)$ surface has a rectangular symmetry and is composed of zigzag rows of atoms that run in the [110] direction. The Ge(110) surface reconstructs into rather large unit cells, which are very complex and involve several atomic layers. The most common surface reconstructions of $\mathrm{Ge}(110)$ are the $(16 \times 2)$ and $\mathrm{c}(8 \times 10)$ reconstructions, which are both composed of five-membered atom rings, hereafter referred as pentagons and feature small (17 151$)$ facets at the steps. These structural models of the $(16 \times 2)$ and $c(8 \times 10)$ have been put forward by Ichikawa ${ }^{19,20}$ are consistent with existing scanning tunneling microscopy data.

Here, in this chapter, we present a combined scanning tunneling microscopy and spectroscopy (STM/STS) and low energy electron microscopy (LEEM) study of the formation of nanowires after deposition of $\mathrm{Pt}$ on $\mathrm{Ge}(110)$. Using LEEM the formation of germanium-platinum eutectic droplets at elevated temperatures will be studied. Upon cooling these eutectic droplets exhibit spinodal decomposition into a Ge-Pt phase and a pure Ge phase will occur. The latter phase segregates to the surface of the solidified droplets and forms a buckled honeycomb lattice, which will study in detail in the next chapter.

\subsection{Experimental details}

Experiments were performed with an Omicron room temperature scanning tunneling microscope (STM-1) operating in ultra-high vacuum (UHV) chamber with a base pressure below $5 \times 10^{-11}$ mbar. The Ge(110) samples were cut from nominally flat, single-side-polished n-type substrates. Samples were mounted on Mo holders and contact to any other metal during preparation and experiment was carefully avoided. The Ge(110) samples have been degassed for about 24 hours at $700 \mathrm{~K}$ and subsequently cleaned by the method described in detail in ref. ${ }^{34}$ This cleaning method involves several cycles of Argon ion bombardment followed by annealing at temperatures of $1100( \pm 25) K$. After five to seven of these cleaning cycles the $\mathrm{Ge}(110)$ samples were atomically clean. Subsequently, Pt is deposited on Ge(110) at room temperature. $\mathrm{Pt}$ is evaporated by resistively heating a $\mathrm{W}$ wire wrapped with 
high purity $\mathrm{Pt}(99.995 \%)$. After Pt-deposition the sample is annealed at $1100( \pm 25)$ $K$ for 5 minutes and subsequently cooled down to room temperature before we placed it into the STM for observation. LEEM experiments were performed with an Elmitec LEEM-III microscope with a base pressure of $5 \times 10^{-11} \mathrm{mbar}$. In that case the sample is heated by e-beam bombardment.

All the STM observations are performed in constant-current mode with an electrochemically etched tungsten tips.

\subsection{Nanowire formation}

After deposition of a sub-monolayer amount of $\mathrm{Pt}$ on $\mathrm{Ge}(110)$ and annealing at $1100 \mathrm{~K}$, self-organized nanowires are formed. A large scale filled-state STM image is shown in Figure 4.1(A). The orientation of the nanowires is along the [11̄0] direction. The nanowires can be several hundred nanometers long and basically cover the whole terrace. Small scale high-resolution images for different Pt coverages are shown in Figure 4.1(B)-(F). As can been seen in Figure 4.1(B) a low coverage of Pt leads to a few nanowires, whereas most of the surface resembles the bare and reconstructed Ge(110) surface, as is evidenced by the omnipresence of pentagons. The nanowires consist of a twin row of double lobed protrusions. These double lobed protrusions occur in-phase as well as out-of-phase registry. There is no long-range ordering along the nanowires due to the presence of defects, phase shifts and missing atoms. The troughs have a depth of $\sim 5 \AA$, suggesting that several atomic layers are involved.

At a slightly higher Pt coverage, large nanowire domains are formed, which basically cover the substrate terraces completely. A high resolution magnification of these nanowires is shown in Figure 4.1(C). In the troughs, in between the nanowires, pentagons are observed. As we show later, these pentagons have an electronic structure that resembles the Ge pentagons of the bare Ge(110) very well, and therefore we tentatively propose that these pentagons consist of Ge atoms.

The distance between adjacent nanowires in Figure 4.1(C) is $3.4 \pm 0.1 \mathrm{~nm}$ (measured from center to center, see the line profile in Figure 4.1(G)), which equals 6 times the surface lattice constant $a_{0}(=5.67 \AA)$ of the $\mathrm{Ge}(110)-(1 \times 1)$ unit cell along the [001] direction. In Figure 4.1(D) regions with NWs with a separation of $3.4 \mathrm{~nm}$ coexist with a regions with a smaller nanowires separation of $2.0 \pm 0.1 \mathrm{~nm}$. Note 
that the separation of $2.0 \mathrm{~nm}$ does not correspond to an integer multiple of $a_{0}$. The $3.4 \mathrm{~nm}$ wide troughs contain pentagons, whereas the $2.0 \mathrm{~nm}$ wide troughs are completely pentagon free. In Figure 4.1(E) only nanowires that are $2.0 \mathrm{~nm}$ spaced apart are found. A line profile along the green line is shown in Figure 4.1(H). The apparent height as extracted from Figure $4.1(\mathrm{H})$ is $2.5 \AA$, i.e. only half of the value found

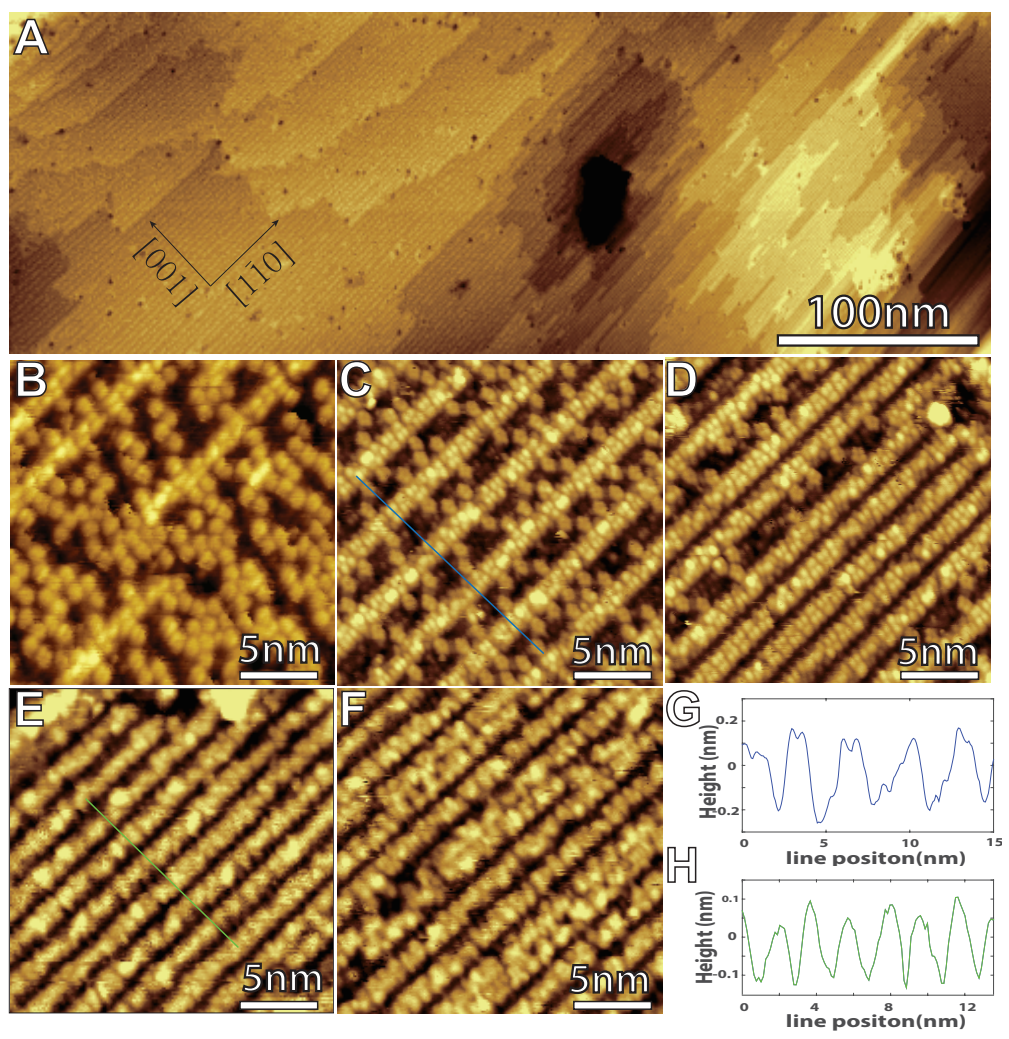

Figure 4.1: (A) Overview STM image (set points $-1.45 \mathrm{~V}, 0.52 \mathrm{nA}$ ) with nanowires oriented along the $[1 \overline{1} 0]$ direction. Note the difference in the density of the nanowires at the right and left hand side of the image;

(B)-(F) STM images of Pt induced nanowires with increasing nanowire density.

Set points are B: $-1.26 \mathrm{~V}, 0.24 \mathrm{nA},(\mathrm{C}):-1.5 \mathrm{~V}, 0.5 \mathrm{nA}$; (D): $-1.2 \mathrm{~V}, 0.5 \mathrm{nA}$; (E): $-1.5 \mathrm{~V}, 0.5 \mathrm{nA} ;(\mathrm{F}):-1.9 \mathrm{~V}, 0.5 \mathrm{nA}$.

$(\mathrm{G})$ and $(\mathrm{H})$ : line profiles along the blue and green lines in $(\mathrm{C})$ and $(\mathrm{E})$, respectively. 
in Figure 4.1(G). It is very likely that this is due to the fact that the STM tip has only limited access to the bottom of the narrow troughs. In Figure 4.1(F) nanowires separations of $1.1 \mathrm{~nm}$ and $2.0 \mathrm{~nm}$ are found. A separation of $1.1 \mathrm{~nm}$ corresponds to $2 a_{0}$. Although the density of the nanowires in Figure 4.1(B)-(F) is clearly different, the structure of the nanowires seems to be unchanged. The latter is in marked contrast to the $\mathrm{Pt} / \mathrm{Si}(110)$ system, where the structure of the Pt induced nanowires depends on the Pt coverage, i.e. on the nanowire separation. ${ }^{26}$

In order to obtain more information on the chemical composition of the Pt-induced nanowires scanning tunneling spectroscopy measurements were performed. Figure 4.2 shows the differential conductivity, $d I / d V$, measured at nanowires that are spaced $3.4 \mathrm{~nm}$ and $2.0 \mathrm{~nm}$ apart, respectively. The differential conductivities were obtained by numerically differentiating of the current-voltage traces $I-V$ curves. The dasheddotted red curve in Figure 4.2(A) is measured on top of the pentagons, which are located in the troughs. The $d I / d V$ measured in the 'empty' space in the troughs is shown by the dotted green lines, while the $d I / d V$ curves measured at the center and the edges of the NWs are represented by the solid black and dashed blue lines,
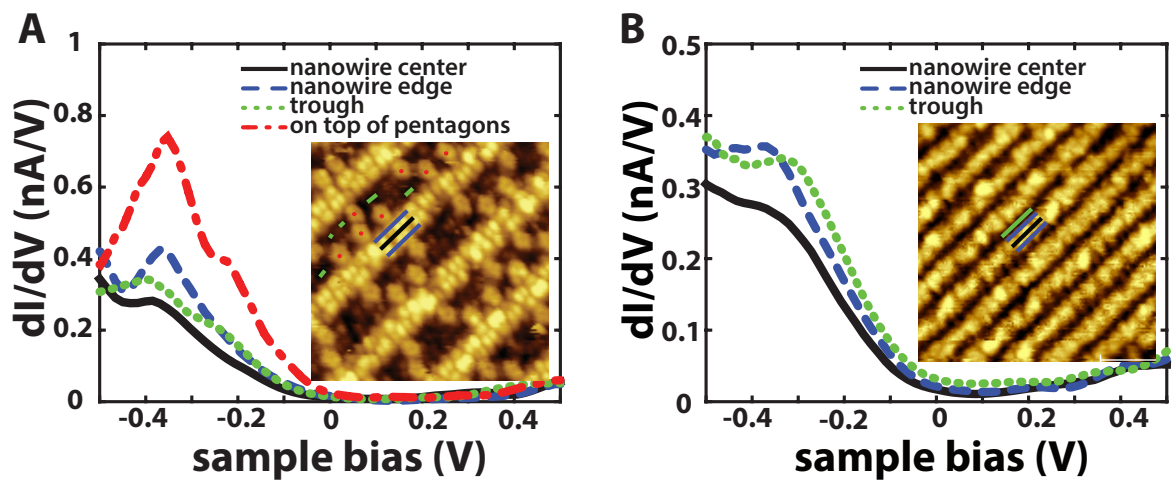

Figure 4.2: (A), (B) Average differential conductance-voltage ( $d I / d V)$ curves measured on areas with different nanowires densities. The black solid lines are measured on the nanowire center, the blue dashed lines are measured above the nanowire edges, the green dotted lines are measured above the 'empty' space in the troughs, while the red dashed-dotted line is measured above the pentagons in the troughs. The spectroscopy set points are both $-1.21 \mathrm{~V}, 0.5 \mathrm{nA}$. 
respectively. The overall appearance of the $d I / d V$ curves in Figure 4.2 is similar, except for a distinct peak at $-0.35 \mathrm{eV}$ in the curves taken on top of the pentagons. For the bare $\mathrm{Ge}(110)$ surface a peak in the density of states around $-0.3 \mathrm{eV}$ is reported. This peak is related to the Ge-pentagons on the Ge(110) surface. ${ }^{35}$ Based on these resemblance we propose that the pentagons that are located in troughs between the nanowires are composed of germanium atoms. The various differential conductivity spectra shown in Figure 4.2(A) and (B) are actually very similar, suggesting that also the Pt-induced nanowires are composed of germanium atoms. This makes sense, since $\mathrm{Pt}$ atoms strive to increase its location coordination number.

In case of the closely related Pt induced nanowires on Si(110) Park et al. observed a singularity in the density of states around $-0.2 \mathrm{eV}$, which is ascribed to Rashba-type surface bands, in connection with a characteristic dip at $-0.3 \mathrm{~V}$, which is assigned to crossing of the splitted bands. ${ }^{29}$ In the dashed blue and dotted green lines of Figure 4.2(B) a weak dip $(\sim-0.4 \mathrm{eV})$ and a small peak $(\sim-0.3 \mathrm{eV})$ can be observed. However, our room temperature STS data lacks sufficient resolution to resolve the exact spatial origin of these features, and hence additional measurements are needed to resolve the origin of these electronic states.

\subsection{D clusters}

Next to the nanowires formation, the formation of $3 \mathrm{D}$ clusters was observed for higher Pt amounts $(2 \sim 3 \mathrm{ML})$. We used LEEM to study the formation of this 3D clusters. A sample was annealed at $1040 \mathrm{~K}$, which is above the eutectic temperature for the Ge-Pt system three-dimensional $\mathrm{Pt}_{0.22} \mathrm{Ge}_{0.78}$ droplets are formed. ${ }^{35-37}$ Figure 4.3(A)-(D) shows snapshots from a LEEM movie during heating and subsequent cooling down cycle. When the temperature is increased to about $943 \mathrm{~K}$, droplets are visible on the surface in Figure 4.3(A). Upon further heating the droplets grow by coalescence resulting into a decrease of the number density of droplets (Figures 4.3(B) and (C)). A decrease of the temperature of the eutectic $\mathrm{Pt}_{0.22} \mathrm{Ge}_{0.78}$ droplets leads to decomposion into a $\mathrm{Ge}_{2} \mathrm{Pt}$ and a pure Ge phase (spinodal decomposition). Ge segregates to the surface of the solidifying clusters. The core of these clusters are composed of a $\mathrm{Ge}_{2} \mathrm{Pt}$, whereas the shell consist of germanium. Scanning tunneling microscopy and spectroscopy measurements of the outermost layer reveal a slightly buckled honeycomb lattice with the germanene lattice constant, which we 
will discuss in detail in next chapter. During the motion of the droplets across the surface they leave, just before they solidify, a trail of nanowire patches behind (see Figure 4.3(D)). In Figures 4.3(E) and (F) a large and small scale STM image of these nanowire bundles are depicted, respectively. The structure of these long nanowire bundles is very similar to the structure observed by Visikovskiy et al. for low Pt coverages on $\mathrm{Si}(110) .{ }^{26}$

\subsection{Conclusions}

We have investigated the structure and electronic properties of Pt induced nanowires on $\mathrm{Ge}(110)$ by STM and STS at room temperature. Three types of nanowires arrays were formed with spacing of $3.4 \mathrm{~nm}\left(6 a_{0}\right), 2.0 \mathrm{~nm}$ and $1.1 \mathrm{~nm}\left(2 a_{0}\right)$, respectively. STS data revealed an electronic state at $-0.35 \mathrm{eV}$, which is attributed to the $\mathrm{Ge}$
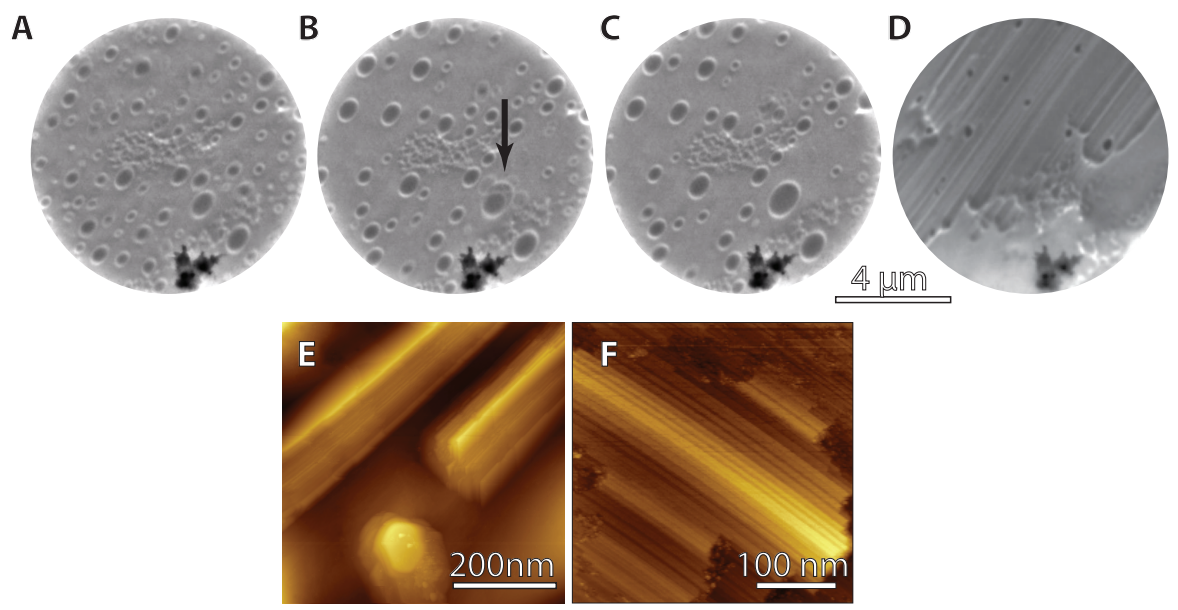

Figure 4.3: Snapshots from a LEEM movie (electron energy $0.5 \mathrm{eV}$, time lapse from (A)-(C): $\sim 10$ seconds) during heating and subsequent cooling. The temperatures in (A)-(D) are $943 K, 967 K, 967 K$ and $376 K$. The black feature in the bottom of the images is due to a defect in our detector. The black arrow in B points to a coalescence event of 4 individual droplets caught in action. STM images of the long nanowire features in (D) are shown in (E) (set point $-0.5 \mathrm{~V}, 0.2 \mathrm{nA}$ ) and (F) ( set point $-1.3 \mathrm{~V}, 0.6 \mathrm{nA}$ ) 
pentagons that are present in the nanowires troughs. At higher Pt coverages and annealing at $1040 \mathrm{~K}$ eutectic Ge-Pt droplets were formed. Upon cooling down 3D nanocrystals and patches with long nanowires with an irregular height and structure are found. 


\section{Bibliography}

[1] Nogami, J., Park, S.-i. \& Quate, C. F. Indium-induced reconstructions of the $\mathrm{Si}(111)$ surface studied by scanning tunneling microscopy. Physical Review $B$ 36, 6221 (1987).

[2] Oncel, N. et al. Quantum confinement between self-organized Pt nanowires on Ge(001). Physical Review Letters 95, 116801 (2005).

[3] Gurlu, O., Adam, O. A., Zandvliet, H. J. W. \& Poelsema, B. Self-organized, one-dimensional Pt nanowires on Ge(001). Applied Physics Letters 83, 4610 4612 (2003).

[4] Wang, J., Li, M. \& Altman, E. I. Scanning tunneling microscopy study of self-organized $\mathrm{Au}$ atomic chain growth on $\mathrm{Ge}(001)$. Physical Review B 70, 233312 (2004).

[5] van Houselt, A., Fischer, M., Poelsema, B. \& Zandvliet, H. J. W. Giant missing row reconstruction of $\mathrm{Au}$ on $\mathrm{Ge}(001)$. Physical Review B 78, 233410 (2008).

[6] Stekolnikov, A. A., Bechstedt, F., Wisniewski, M., Schäfer, J. \& Claessen, R. Atomic nanowires on the Pt/Ge(001) surface: Buried Pt-Ge versus top Pt-Pt chains. Physical Review Letters 100, 196101 (2008).

[7] $\mathrm{Ma}, \mathrm{X}$.-D. et al. Strain relief guided growth of atomic nanowires in a $\mathrm{Cu}_{3} \mathrm{~N}$ $\mathrm{Cu}(110)$ molecular network. Physical Review Letters 102, 205503 (2009).

[8] Oncel, N. Atomic chains on surfaces. Journal of Physics: Condensed Matter 20, 393001 (2008).

[9] Lichtenstein, T., Teiken, H., Pfnür, H., Wollschläger, J. \& Tegenkamp, C. Auchains grown on Ge (100): A detailed SPA-LEED study. Surface Science 632, 64-70 (2015).

[10] Niikura, R., Nakatsuji, K. \& Komori, F. Local atomic and electronic structure of Au-adsorbed $\mathrm{Ge}(001)$ surfaces: Scanning tunneling microscopy and X-ray photoemission spectroscopy. Physical Review B 83, 035311 (2011). 
[11] van Weperen, I., Plissard, S. R., Bakkers, E. P., Frolov, S. M. \& Kouwenhoven, L. P. Quantized conductance in an InSb nanowire. Nano Letters 13, 387-391 (2012).

[12] Do, E. H. \& Yeom, H. W. Electron quantization in broken atomic wires. Physical Review Letters 115, 266803 (2015).

[13] Churchill, H. O. et al. Toward single atom chains with exfoliated tellurium. Nanoscale Research Letters 12, 488 (2017).

[14] Vanpoucke, D. E. P. Modeling 1D structures on semiconductor surfaces: synergy of theory and experiment. Journal of Physics: Condensed Matter 26, 133001 (2014).

[15] Kane, C. L. \& Fisher, M. P. A. Transport in a one-channel Luttinger liquid. Physical Review Letters 68, 1220 (1992).

[16] Luttinger, J. M. An exactly soluble model of a many-fermion system. Journal of Mathematical Physics 4, 1154-1162 (1963).

[17] Losio, R. et al. Band splitting for $\mathrm{Si}$ (557)-Au: Is it spin-charge separation? Physical Review Letters 86, 4632 (2001).

[18] Jompol, Y. et al. Probing spin-charge separation in a Tomonaga-Luttinger liquid. Science 325, 597-601 (2009).

[19] Ichikawa, T. An ab initio study on the atomic geometry of reconstructed Ge(110) 16× 2 surface. Surface Science 544, 58-66 (2003).

[20] Ichikawa, T. Atomic geometry of the Ge(110) c $(8 \times 10)$ structure. Surface Science 560, 205-212 (2004).

[21] Mocking, T. F., Bampoulis, P., Oncel, N., Poelsema, B. \& Zandvliet, H. J. W. Electronically stabilized nanowire growth. Nature Communications 4 (2013).

[22] He, Z., Stevens, M., Smith, D. J. \& Bennett, P. A. Dysprosium silicide nanowires on Si(110). Applied Physics Letters 83, 5292-5294 (2003).

[23] He, Z., Smith, D. J. \& Bennett, P. A. Endotaxial silicide nanowires. Physical Review Letters 93, 256102 (2004). 
[24] Bhattacharjee, K., Roy, A., Kundu, K. \& Dev, B. N. Electronic structure of Ag-adsorbed nanowire-like stripes on $\operatorname{Si}(110)-(16 \times 2)$ surfaces. I. An in situ STM and STS experiment. Physical Review B 77, 115430 (2008).

[25] Kang, S. H., Kim, K. S. \& Yeom, H. W. Electronic structure of Au-induced surface phases on $\mathrm{Si}(110)$ : LEED and angle-resolved photoemission measurements. Physical Review B 78, 075315 (2008).

[26] Visikovskiy, A., Yoshimura, M. \& Ueda, K. Pt-induced structures on Si(110) studied by STM. Applied Surface Science 254, 7626-7629 (2008).

[27] Visikovskiy, A., Yoshimura, M. \& Ueda, K. Initial stages of platinum silicide formation on $\mathrm{Si}(110)$ studied by scanning tunneling microscopy. Japanese Journal of Applied Physics 48, 08JB11 (2009).

[28] Hong, I.-H., Tsai, Y.-F. \& Chen, T.-M. Self-organization of mesoscopically ordered parallel gd-silicide nanowire arrays on a $\operatorname{Si}(110)-16 \times 2$ surface: A massively parallel active architecture. Applied Physics Letters 98, 193118 (2011).

[29] Park, J. et al. Self-assembled nanowires with giant Rashba split bands. Physical Review Letters 110, 036801 (2013).

[30] Hus, S. M. \& Weitering, H. H. Formation of uni-directional ultrathin metallic $\mathrm{YSi}_{2}$ nanowires on Si(110). Applied Physics Letters 103, 073101 (2013).

[31] Zou, Z.-Q., Shi, G.-M., Sun, L.-M. \& Liu, X.-Y. Manganese nanoclusters and MnSi 1.7 nanowires formed on $\mathrm{Si}(110)$ : A comparative x-ray photoelectron spectroscopy study. Journal of Applied Physics 113, 024305 (2013).

[32] Mohottige, R. N. \& Oncel, N. Iridium-silicide nanowires on Si(110) surface. Surface Science 641, 237-241 (2015).

[33] Feng, J. et al. P-channel germanium FinFET based on rapid melt growth. Electron Device Letters, IEEE 28, 637-639 (2007).

[34] Zandvliet, H. J. W. The Ge(001) surface. Physics Reports 388, 1-40 (2003). 
[35] Bampoulis, P. et al. Germanene termination of ge ${ }_{2}$ pt crystals on $\mathrm{Ge}(110)$. Journal of physics: Condensed matter 26, 442001 (2014).

[36] Safaei, A., Poelsema, B., Zandvliet, H. J. W. \& van Gastel, R. Spinodal decomposition driven formation of Pt-nanowires on Ge(001). New Journal of Physics 16, 113052 (2014).

[37] Zhang, L., Bampoulis, P., van Houselt, A. \& Zandvliet, H. J. W. Twodimensional Dirac signature of germanene. Applied Physics Letters 107, 111605 (2015). 



\section{Germanene termination of Ge-Pt crystals on $\mathrm{Ge}(\mathbf{1 1 0})$}

The deposition of several monolayers of Pt on Ge(110) followed by annealing at $1100 \mathrm{~K}$ results in the formation of $3 \mathrm{D}$ metallic Pt-Ge nanocrystals. The outermost layer of these crystals exhibits a honeycomb structure. We propose that the outermost layer of the germanium-platinum nanocrystal is germanene. The differential conductivity of the interior of the germanene sheet has a V-shape, which is the hallmark of the two-dimensional Dirac system. The zigzag edges of germanene are reconstructed and display a $4 \times$ periodicity. Near the reconstructed germanene zigzag edges the shape of the differential conductivity changes from a $V$-shape to a more parabolic-like shape. 


\subsection{Introduction}

In 2004 Novoselov and Geim ${ }^{1}$ ignited a revolution in materials science by preparing graphene which consists of a single layer of $\mathrm{sp}^{2}$ hybridized carbon atoms that are arranged in a planar honeycomb registry. The charge carriers in graphene behave as relativistic massless particles that are described by the Dirac equation, i.e. the relativistic variant of the Schrödinger equation. In the vicinity of the Dirac point the dispersion relation is linear, i.e. $E=v_{F} \hbar k$, where $v$ is the Fermi velocity, $\hbar$ the reduced Planck constant and $k$ the wave vector. Graphene is a semimetal and the density of states scales linearly with energy. One of the interesting properties of finite graphene sheets is the existence of electronic states that are localized at the edges of graphene. Theory predicts that a zigzag terminated graphene edge is metallic, whereas an armchair terminated graphene edge is semiconducting. ${ }^{2-4}$ Scanning tunneling microscopy and spectroscopy studies of zigzag and armchair monatomic step edges of graphite have indeed confirmed these theoretical predictions. ${ }^{5,6}$

Since the rise of graphene there has been a growing interest in other two-dimensional materials that exhibit 'graphene'-like properties. The most obvious alternatives for graphene are the group IV elements, i.e. silicon, germanium and tin, might exhibit similar properties as graphene. Already in 1994 Takeda and Shiraishi ${ }^{7}$ performed quantum mechanical ab initio calculations on planar silicon and germanium structures that have the graphite structure. They pointed out that the lowest energy configuration was obtained if the two atoms of the honeycomb are slightly displaced with respect to each other in a direction normal to the planar structure. Their calculations also revealed that these buckled Si and Ge structures exhibited semi-metallic properties. Unfortunately, they did not pay any attention to the exact $\mathrm{k}$-dependence of the energy dispersion relations in the vicinity of the Fermi level. More than a decade later Guzmán-Verri and Lew Yan Voon ${ }^{8,9}$ showed, using tight binding calculations, that a 2D silicon sheet with the graphite structure has Dirac cones. Hence the electrons in these 2D silicon sheets behave as massless Dirac fermions. The Si and Ge analogues of graphene are referred to as silicene and germanene, respectively. First-principles calculations by Cahangirov et al. ${ }^{9}$ revealed that a single sheet of germanium atoms with a honeycomb structure is also stable. The freestanding germanene honeycomb lattice is not fully planar, but buckled. The two hexagonal sub-lattices of the honeycomb lattice are displaced vertically by 0.64 
$\AA$, which is slightly larger than the buckling in silicene $(0.44 \AA)$. The buckling results into a weaker $\pi$ bonding and the perpendicular $p_{z}$ orbital hybridizes with the in-plane orbitals. Similar to graphene and silicene the $\pi-\pi^{*}$ band crossings at the $K$ and $K^{\prime}$ points of the hexagonal Brillouin zone occur at the Fermi level. In the vicinity of the Fermi level the $E(k)$ dispersion relations are linear and therefore the charge carriers behave as massless Dirac fermions. The calculated Fermi velocity of germanene is, however, smaller than the Fermi velocity of graphene. Due to germanium's large atomic number the spin-orbit coupling is rather large. The spin-orbit gap of the $\pi$ orbitals of germanene at the Dirac points is $23.9 \mathrm{meV},{ }^{10}$ which is substantially larger than the spin-orbit gaps of graphene $(<0.05 \mathrm{meV})$ and silicene $(1.55$ $\mathrm{meV})$. The large spin-orbit gap makes germanene an excellent candidate to exhibit the quantum spin Hall effect. ${ }^{10}$ The existence of this quantum spin Hall effect was put forward by Kane and Mele. ${ }^{11}$ The quantum spin Hall effect state is characterized by a bulk gap and conducting spin-polarized edge states without dissipation at the sample boundaries. The quantum spin Hall effect in graphene is very difficult to access because of the required extremely low temperatures. In the case of germanene, however, the quantum spin Hall effect should occur near room temperature. In contrast to graphene, silicene and germanene do not occur in nature and therefore these materials have to be synthesized.

Silicene and silicene nanoribbons have already been grown on $\operatorname{Ag}(110), \operatorname{Ag}(111)$, $\mathrm{ZrB}_{2}(0001)$ and $\operatorname{Ir}(111)$ surfaces, ${ }^{12-18}$ but to the best of our knowledge there are only a few papers that report the growth of germanene. ${ }^{19-22}$ Dávila et al. ${ }^{20}$ have studied the growth germanene on Au (111) using scanning tunneling microscopy, synchrotron radiation core-level spectroscopy and density functional theory calculations. Li et al. ${ }^{19}$ have grown a single layer of germanium on a $\operatorname{Pt}(111)$ substrate and subsequently analyzed this germanium sheet with low energy electron diffraction and scanning tunneling microscopy. The low energy electron diffraction pattern reveals a $(\sqrt{1} 9 \times \sqrt{19})$ reconstruction that is slightly rotated with respect to the underlying $\mathrm{Pt}(111)$ lattice. These observations are confirmed by scanning tunneling microscopy images, albeit Li et al. ${ }^{19}$ did not manage to achieve atomic resolution. However, in a recent paper by Švec et al. ${ }^{23}$ it was suggested that the Ge induced $(\sqrt{1} 9 \times \sqrt{1} 9)$ reconstruction on $\mathrm{Pt}(111)$ is in fact a surface alloy composed of $\mathrm{Ge}_{3} \mathrm{Pt}$ tetramers that resembles a twisted kagome lattice.

In this chapter we study a new synthesis method of germanene by growing $\mathrm{Pt}$ 
on a $\mathrm{Ge}(110)$ substrate. The deposition of a few monolayers of Pt on $\mathrm{Ge}(110)$ followed by annealing at temperatures around $1100 \mathrm{~K}$ leads to the formation of these 3D nanocrystals on the Ge(110) substrate. These 3D crystals are terminated by a buckled honeycomb structure, which we interpret as germanene. The structural and electronic properties of germanene sheets that are found on 3D nanocrystals are studied further. We will show that the density of states of these germanene sheets hints to a 2D Dirac system, albeit the density of states does not completely vanish at the Fermi level. We will also study the structural properties as well as the density of states of the edges of the germanene sheets. We found zigzag edges of germanene that exhibit a $4 \times$ periodicity. Surprisingly, we did not find any evidence for the existence of a metallic edge state.

\subsection{Experimental details}

Experiments have been performed with a scanning tunneling microscope operating in ultra-high vacuum. The $\mathrm{Ge}(110)$ samples are cut from nominally flat $10 \times 10 \times 0.5$ $\mathrm{mm}^{3}$, singleside polished n-type substrates. Samples are mounted on Mo holders and contact of the samples to any other metal during preparation and experiment has been carefully avoided. The Ge(110) samples have been cleaned with the same method as descriped in previous chapters. This method involves several cycles of Argon ion sputtering at $500 \sim 800 \mathrm{eV}$ and annealing at 1100 ( \pm 25 ) $\mathrm{K}$. After checking the cleanliness and flatness of the substrate with scanning tunneling microscopy $\mathrm{Pt}$ was deposited onto the substrate at room temperature. Pt was evaporated by resistively heating a W wire wrapped with high purity $\mathrm{Pt}$ (99.995\%). After Pt-deposition the sample was shortly annealed at $1100( \pm 25) K$ and subsequently cooled slowly to room temperature before placing it into the scanning tunneling microscope for imaging. Tapping mode atomic force microscopy (AFM) was performed using an Agilent 5100 atomic force microscope (Agilent) and HI'RES-C14/CR-AU probes (MikroMasch), with a nominal spring-constant of $5 \mathrm{~N} / \mathrm{m}$ and resonance frequency of $160 \mathrm{kHz}$. 


\subsection{D Ge-Pt nanocrystals}

In Chapter 3 the structral properties of the bare $\mathrm{Ge}(110)$ surface have been present. In contrast to its low index counterparts the unreconstructed $\mathrm{Ge}(110)$ is anisotropic. The bulk terminated Ge(110) surface consists of atomic trenches that run in the [110] direction. The clean and reconstructed $\mathrm{Ge}(110)$ surface is well-documented and exhibits $(16 \times 2)$ and $c(8 \times 10)$ reconstructed domains as well as disordered domains. The elementary building block of all these reconstructions is a pentagon, a five-membered $\mathrm{Ge}$ atom ring. In Figure 5.1(A) a scanning tunneling microscopy image taken after the deposition of $2 \sim 3$ monolayers of Pt and annealing at 1100 $K$ is depicted. The surface is composed of flat regions (ii) and 3D crystals (i). In Figure 5.1(B) a scanning tunneling microscopy image recorded on-top of one of the 3D crystals is depicted. The step edges are very straight and their heights are quantized in units of $5.6 \pm 0.6 \AA \quad$ (see Figure 5.1(D)). Scanning tunneling spectra recorded on both regions reveal that the (ii) regions are semiconducting, whereas the (i) regions are metallic (see Figure 5.1(C)). It is important to point out that the formation of these 3D crystals only occurs upon annealing at temperatures higher than $1000 \sim 1100 K$. The phase diagram of Ge-Pt system exhibits an eutectic alloy at $1043 \mathrm{~K}$. This occurs at a composition of $22 \%$ and $78 \% \mathrm{Pt}$ and Ge, respectively. Low energy electron microscopy (LEEM) images show that slightly above this temperature liquid drops are formed and move as large entities across the surface. ${ }^{24}$

The eutectic droplets cover approximately $5 \%$ of the surface. Upon cooling down these droplets solidify and spinodal decomposition must be expected to occur. Ideally, two stable coexisting phases will form, which are most close to the eutectic composition. The (bulk-) phase diagram identifies a low density or even platinumfree phase and an ordered $\mathrm{Ge}_{2} \mathrm{Pt}$ phase. We anticipate that the cluster (i) in Figure 5.1(B) is actually a $\mathrm{Ge}_{2} \mathrm{Pt}$ crystallite. Interestingly, the shape of the Ge-Pt crystal is variable. In Figure 5.2 large scale images of a pyramidal shaped and a flat-topped $\mathrm{Ge}_{2} \mathrm{Pt}$ crystals are shown. The elongated $\mathrm{Ge}_{2} \mathrm{Pt}$ crystal has a length of $1.6 \mu \mathrm{m}$, a width of $0.5 \mu \mathrm{m}$ and a height of $80 \mathrm{~nm}$. The flat-topped $\mathrm{Ge}_{2} \mathrm{Pt}$ crystals are terminated by a buckled honeycomb lattice with a lattice constant of $4.2 \pm 0.2 \AA$ (see Figure 5.3(A) for a small scale scanning tunneling microscopy image). The buckled honeycomb lattice is composed of two triangular sub-lattices that are displaced with 
respect to each other in a vertical direction by $0.2 \AA$. This buckling is significantly smaller than the $0.6 \sim 0.7 \AA$ predicted for germanene by Cahangirov et al. ${ }^{9}$ and Garica et al. ${ }^{25}$ using density functional theory calculations. However the nearestneighbor distance between the atoms of the honeycomb lattice is $2.5 \pm 0.1 \AA$, i.e. very close to the predictions for free-standing germanene. ${ }^{9,25}$

To obtain more information we decided to have a more detailed look at the struc-
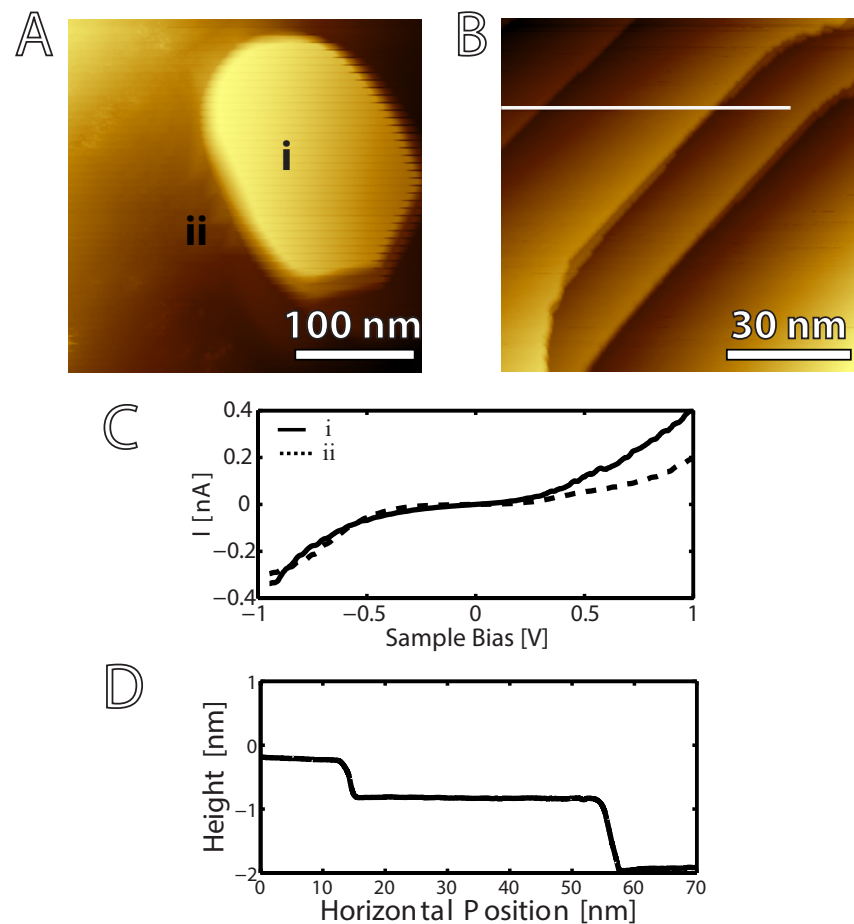

Figure 5.1: (A) Scanning tunneling microscopy image after deposition and annealing at $1100 \mathrm{~K}$ of $\mathrm{Pt}$ on $\mathrm{Ge}(110)$. Sample bias $-1.0 \mathrm{~V}$ and tunneling current 0.5 $\mathrm{nA}$ (please note that the regularly spaced lines on the cluster are due to the grid $I-V$ scan). The height of the cluster is $\sim 20 \mathrm{~nm}$ and the aspect ratio 3:4.

(B) Scanning tunneling microscopy image recorded on the cluster (region i) shown in (A). Sample bias $-1.4 \mathrm{~V}$ and tunneling current $0.5 \mathrm{nA}$.

(C) $I$ - $V$ curves recorded on regions (i) and (ii). Set points: Sample bias $-1.0 \mathrm{~V}$ and tunneling current $0.5 \mathrm{nA}$. (D) Line scan taken across the step edges in (B). 


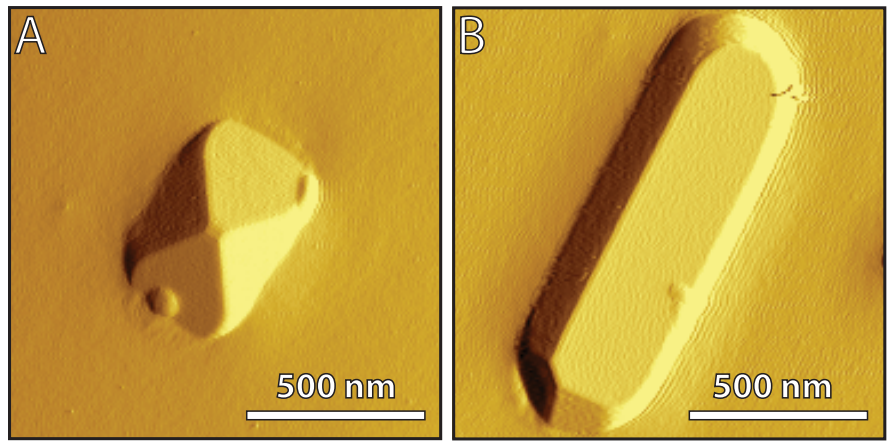

Figure 5.2: (A) Large scale atomic force microscopy image of a pyramidal shaped $\mathrm{Ge}_{2} \mathrm{Pt}$ crystal. The height of the cluster is $105 \mathrm{~nm}$.

(B) Large scale atomic force microscopy image of a flat-topped $\mathrm{Ge}_{2} \mathrm{Pt}$ crystal. The height of the cluster is $80 \mathrm{~nm}$.

ture of the crystallites' outermost layer. A well-ordered honeycomb lattice, which consists of two hexagonal sub-lattices is resolved. In Figure 5.3(A) filled state scanning tunneling microscopy image (3D representation) is shown. Scanning tunneling spectra are recorded on the atoms of the honeycomb, as well as in the center of the honeycomb (see Figure 5.3(B)). Both spectra are recorded at the same set point and it is evident that the center of the honeycomb is more metallic than the atoms of the honeycomb. The height difference between the brightest and dimmest atom of the honeycomb is $0.2 \AA$, the nearest-neighbor distance is $2.5 \pm 0.1 \AA$ and the honeycomb lattice constant is $4.2 \pm 0.2 \AA$. Aside from the fact that these lattice parameters are in favor of germanene we would like to emphasize that Pt tends to maximize its total number of neighbors and therefore the honeycomb lattice cannot consist of Pt atoms alone.

Our findings are intimately in line with a structural model published by Xie and Nesper ${ }^{26}$ for $\mathrm{SrGe}_{1.2} \mathrm{Si}_{0.8}$. They found a hexagonal (P6/mmm) structure with lattice vectors $a=0.436 \mathrm{~nm}$ and $c=0.454 \mathrm{~nm}$. Very similar values were obtained for $\mathrm{CaSi}_{1.2} \mathrm{Zn}_{0.8}$. A model of this structure is shown in Figure 5.3(C): the metal atoms ( $\mathrm{Sr}, \mathrm{Ca})$ are located in one plane in between planes with the semiconductor atoms with a honeycomb structure. The atomic density of the layer of semiconductor atoms (two atoms per honeycomb unit cell) is twice the atomic density of the 
Pt layer (one atom per honeycomb unit cell, see Figure 5.3(C)). The Ge layer is the terminating plane with its lower surface free energy and the natural tendency of the metal $(\mathrm{Pt})$ atoms to search for the highest coordination. The $I-V$ curves measured in the center of the honeycomb mainly probe the second $(\mathrm{Pt})$ layer, while the $I-V$

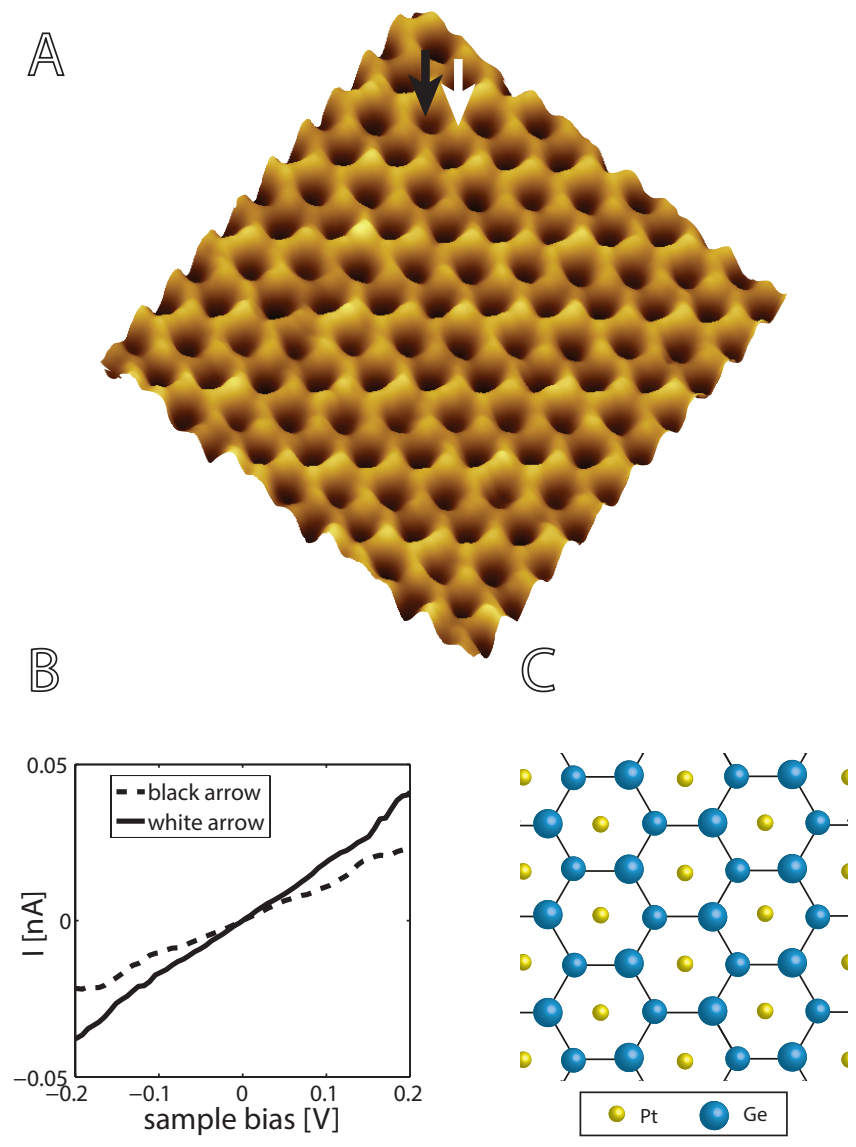

Figure 5.3: (A) Scanning tunneling microscopy image of the honeycomb lattice. Sample bias $-0.5 \mathrm{~V}$, tunnel current $0.2 \mathrm{nA}$. Image size $4.5 \mathrm{~nm} \times 4.5 \mathrm{~nm}$.

(B) $I-V$ curve recorded at a bright atom of the honeycomb lattice (dotted line, black arrow) and at the center of the honeycomb (solid line, white arrow).

(C) Schematic ball model. Top layer: buckled honeycomb lattice (germanene, in blue) second layer: platinum (in yellow). 
curves taken at the atoms of the honeycomb probe the top germanene layer (see Figure 5.3(B)). Further support for this attribution is coming from the parameter of the lattice taken from Figure 5.3(A), equaling $4.2 \pm 0.2 \AA$, in perfect agreement with. ${ }^{26}$ From the line profile in Figure 5.1(D) we extract a step height of $0.56 \pm$ $0.06 \mathrm{~nm}$. Note that we also observe a double step in the line profile. Regardless of the termination this determines a $c / a$ ratio of 1.29 , i.e. slightly larger than the value of 1.05 for the cases of. ${ }^{26}$ Since the ionic radius of Pt is smaller than both that of $\mathrm{Ca}$ and of $\mathrm{Sr}$ we conclude that the bond strength of the semiconductor plane and the metallic planes is less strong. Therefore, the terminating semiconductor planes incline towards an enhanced germanene character. This is further reinforced by the fact that, as shown in Figures 5.3(A) and 5.4(A), some atoms of the honeycomb appear a bit brighter than others. At small negative sample biases and positive sample biases it is clear that the honeycomb lattice consists of two hexagonal sub-lattices that are slightly displaced vertically with respect to each other. This is exactly what one would expect for germanene, albeit the observed buckling of $0.2 \AA$ is still substantially smaller than the predicted buckling for free-standing germanene $(0.64$ $\AA$ ). Therefore, we belive we have a strong case for a germanene termination of the (0001) surface of $\mathrm{Ge}_{2} \mathrm{Pt}$ crystallites on $\mathrm{Ge}(110)$.

\subsection{Spectroscopy}

Scanning tunneling spectroscopy was performed on a $13 \mathrm{~nm} \times 13 \mathrm{~nm}$ area. At every point of a 60 by 60 grid an $I-V$ curve was recorded with the feedback loop disabled. The voltage was swept between $-1 \mathrm{~V}$ and $1 \mathrm{~V}$. The set point tunnel current at -1 $\mathrm{V}$ was $0.6 \mathrm{nA}$. The differential conductivity, $d I / d V$, was obtained by numerically differentiating the $I-V$ curves. Subsequently, we took the average of all $3600 d I / d V$ curves (see Figure 5.4(B)). The $d I / d V$ curve, which is proportional to the local density of states, has a well-defined $\mathrm{V}$-shape reminiscent of the density of states of a 2D Dirac system. The slope of the $d I / d V$ curve is, however, a bit more steeper for the unoccupied states than for the occupied states. At first glance this seems at variance with a 2D Dirac system, for which the density of states should be fully symmetric around the Dirac point due to electron-hole symmetry. Two remarks regarding the shape of our $d I / d V$ measurements are in place here. First, our $d I / d V$ spectra are not recorded on a free-standing germanene sheet, but on a germanene sheet on a metal- 
lic $\mathrm{Ge}_{2} \mathrm{Pt}$ support. Second, also the electronic structure of the W tip has an effect on the measured $d I / d V$. The observed asymmetry of the $d I / d V$ curves can therefore also be due to the electronic structure of the underlying $\mathrm{Ge}_{2} \mathrm{Pt}$ substrate and/or W tip. We have investigated several samples and used various STM tips and in virtually all cases we find slightly asymmetric $d I / d V$ curves. In some cases the density of states at energies below the Fermi level was higher than the density of states above the Fermi level, whereas in other cases we observed a reversed asymmetry (see Figure 5.4(C)). However, in all cases the spatial variation of the $d I / d V$ curves within a scanning tunneling spectroscopy grid scan is very uniform. The non-zero value of $d I / d V$ at the minimum of the V-shaped curve is ascribed to the underlying metallic $\mathrm{Ge}_{2} \mathrm{Pt}$ substrate. The energy range of the V-shaped density of states is about $1 \mathrm{eV}$, which is significantly larger than the predicted $0.5 \sim 0.8 \mathrm{eV}$ energy range of the Dirac cone for free-standing germanene. ${ }^{9}$ As a possible explanation for this large energy range we suggest that the electronic states near the $\Gamma$ point of the germanene Brillouin zone are affected by the underlying $\mathrm{Ge}_{2} \mathrm{Pt}$ substrate. As a final remark regarding the shape of the $d I / d V$ spectrum we would like to emphasize that near the minimum of $d I / d V$ curve the shape deviates from a perfect V-shape due to thermal broadening.

Additional support for the linear dispersion relation of our germanene sheet can, in principle, be obtained from an angle-resolved photoemission study. Unfortunately the small size of our $\mathrm{Ge}_{2} \mathrm{Pt}$ crystals $(<1-2 \mu \mathrm{m})$ does not allow us to perform angle-resolved photoemission experiments. This is a pity because these experiments would also allow us to determine the Fermi velocity from the slope of the dispersion curve.

In Figure 5.5(A) a scanning tunneling microscopy image of germanene sheet is shown. Line scans taken across and along the step edge are depicted in Figures 5.5(B) and 5.5(C), respectively. A line scan taken along the step edge reveals a $4 a$ periodicity, where $a=0.42 \mathrm{~nm}$ is the lattice constant of germanene. The edges of germanene can be divided into two types of edges, the so-called zigzag and armchair edges. Although we did not succeed to obtain atomic resolution at the step edges, we determined the orientation of the step edges from atomically resolved images recorded on the flat terraces. Based on our observations we conclude that the step edge in Figure 5.5(A) is a zigzag type of step edge. The unreconstructed zigzag edge consists of a chain of hexagons. However, the zigzag edge of graphene 
can lower its energy by forming a chain of pentagon-heptagon pairs, the so-called Stone-Wales defects. ${ }^{27-29}$ This reconstruction of the zigzag edge is referred to as the $\mathrm{zz}(57)^{27}$ or the Stone-Wales reconstruction. The $\mathrm{zz}(57)$ reconstruction leads to a doubling of the periodicity of the edge. The periodicity of the germanene zigzag edge incture of the zigzag edge. However, it is not difficult to come up with simple structural models that are for instance composed of alternating units of $\mathrm{zz}(57)$ and zz(66) building blocks (see Figure 5.6 for a model of the zz(5766) edge). As a final remark we would like to mention that besides the abundant reconstructed zigzag edges we also found several rough edges (see the inset of Figure 5.5(A) for an example).

Near the step edge the differential conductivity is smaller than at the terraces (see Figure 5.5(D)). The shape of the $d I / d V$ curve has also changed substantially from an asymmetric V-shape to a flatter, more parabolic, shape. Interestingly, our observations are very similar to results obtained by Liu et al. ${ }^{30}$ for graphene sheets on a hexagonal boron nitride layer. In any case the reconstructed germanene zigzag edges do not exhibit a pronounced metallic edge state.

As pointed out by Koskinen, Malola, and Häkkinen ${ }^{27}$ there are two types of metal-
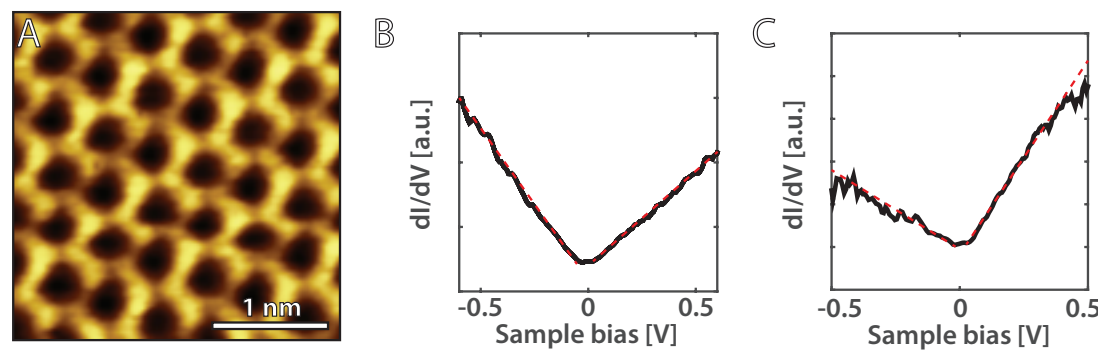

Figure 5.4: (A) Small scale $(3 \mathrm{~nm} \times 3 \mathrm{~nm})$ scanning tunneling microscopy image taken at the flat top plane of one of the germanene terminated $\mathrm{Ge}_{2} \mathrm{Pt}$ crystals. Sample bias $-0.5 \mathrm{~V}$, tunnel current $0.2 \mathrm{nA}$.

(B) Differential conductivity recorded at the interior of a germanene sheet. The $d I / d V$ curve is averaged over a $13 \mathrm{~nm}$ by $13 \mathrm{~nm}$ area using a $60 \times 60$ grid. Set point voltage is $-1 \mathrm{~V}$ and set point tunnel current is $0.6 \mathrm{nA}$.

(C) Differential conductivity recorded at the interior of a germanene sheet. The $d I / d V$ curve is averaged over a $13 \mathrm{~nm}$ by $13 \mathrm{~nm}$ area using a $60 \times 60$ grid. Set point voltage is $-0.54 \mathrm{~V}$ and set point tunnel current is $0.52 \mathrm{nA}$ 
lic zigzag edges states. The zigzag edge of graphene has a metallic edge state that does not stem from the dangling bonds at the edge, but from the topology of the $\pi$-electron network. The energy band of the metallic edge state of the zigzag edge is rather flat resulting in a well-defined peak in the density of states at the Fermi level. The armchair edge does not possess such a metallic edge state. The other metallic edge state comes from the dangling bonds of the edge atoms.

As a final remark we would like to stress that the $\mathrm{Ge}_{2} \mathrm{Pt}$ substrate is not an ideal substrate for germanene because of its metallic character. A band gap material, especially a wide band gap material, such as hexagonal boron nitride (h-BN) or alu-
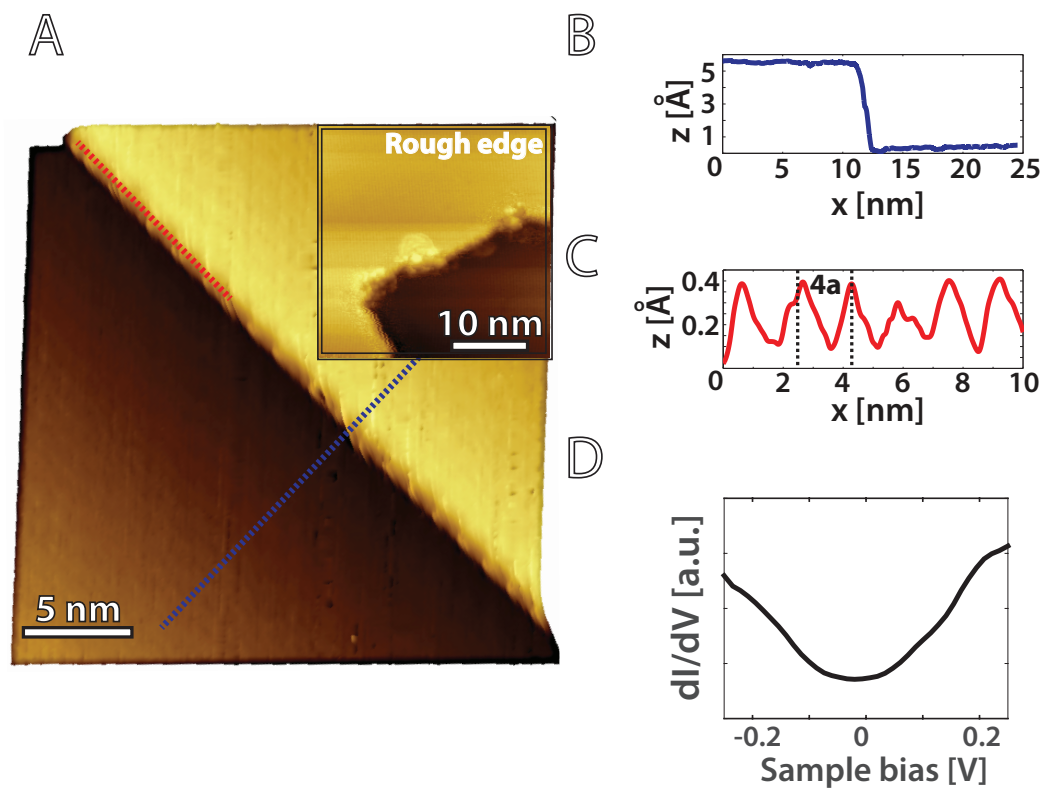

Figure 5.5: (A) Scanning tunneling microscopy image of a germanene step edge (25 $\mathrm{nm} \times 25 \mathrm{~nm}$ ). Inset: scanning tunnel microscopy image of a rough edge.

(B) Line scan taken across the step edge (blue dotted line). The monatomic step height is $0.56 \mathrm{~nm}$.

(C) Line scan taken along the step edge (red dotted line). The periodicity is $4 a$, where $a=0.42 \mathrm{~nm}$ is the lattice constant of germanene

(D) Differential conductivity recorded near the step edge. Set point voltage $-1.57 \mathrm{~V}$ and set point tunnel current $0.47 \mathrm{nA}$. 
minum nitride (AlN), is a much more appealing substrate for germanene. These substrates allow to decouple the important electronic states of the germanene near the Fermi level from the underlying substrate. h-BN is probably the best candidate because the lattice constant of the h-BN lattice is very comparable to the nearestneighbor distance of the germanene lattice.
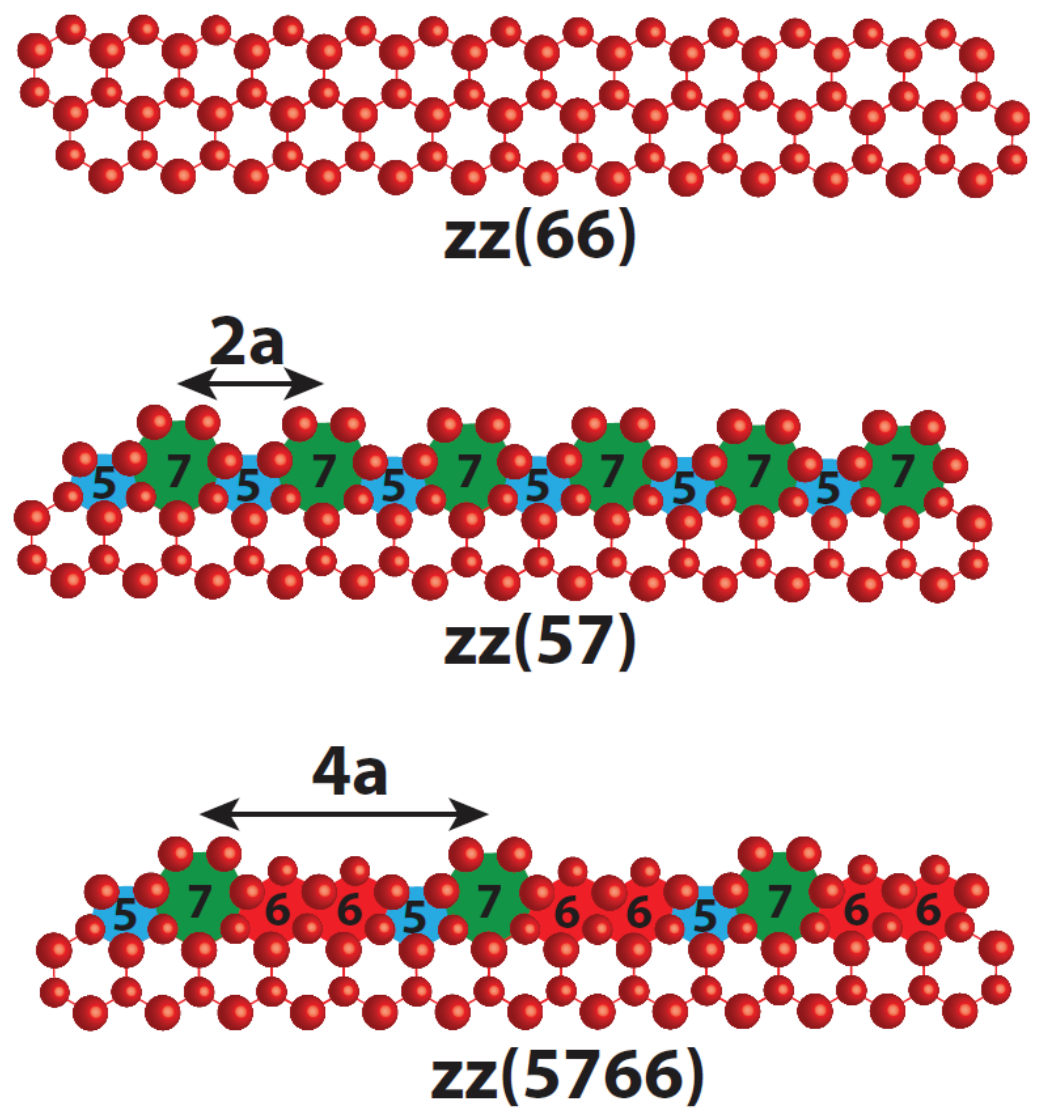

Figure 5.6: Schematic models of an unreconstructed zigzag edge (top panel), a zz(57) edge (middle panel) and a zz(5766) edge (bottom panel). 


\subsection{Conclusions}

In conclusion, the deposition and subsequent annealing of $\mathrm{Pt}$ on $\mathrm{Ge}(110)$ leads to the formation of microscopic Ge-Pt crystals. The outermost layer of these crystals exhibits a honeycomb structure. This honeycomb lattice is composed of two hexagonal sub-lattices that that are displaced vertically by $0.2 \AA$ with respect to each other. The nearest-neighbor distance is $2.5 \pm 0.1 \AA$. Based on our experimental observations we propose that the outermost layer of the Ge-Pt crystals is a germanene layer. The interior of the germanene sheets displays a V-shaped density of states indicative for a 2D Dirac system. However, the density of states at the minimum of the $\mathrm{V}$-shaped curve does not completely vanish, which we ascribe to the underlying metallic Ge-Pt substrate. Two types of step edges are found on the germanene sheets: straight reconstructed zigzag step edges with a $4 \times$ periodicity and rough step edges. Both type of step edges have a parabolic shaped $d I / d V$ curve. 


\section{Bibliography}

[1] Novoselov, K. S. et al. Electric field effect in atomically thin carbon films. Science 306, 666-669 (2004).

[2] Klein, D. J. Graphitic polymer strips with edge states. Chemical Physics Letters 217, 261-265 (1994).

[3] Fujita, M., Wakabayashi, K., Nakada, K. \& Kusakabe, K. Peculiar localized state at zigzag graphite edge. Journal of the Physical Society of Japan 65, 1920-1923 (1996).

[4] Nakada, K., Fujita, M., Dresselhaus, G. \& Dresselhaus, M. S. Edge state in graphene ribbons: Nanometer size effect and edge shape dependence. Physical Review B 54, 17954 (1996).

[5] Niimi, Y. et al. Scanning tunneling microscopy and spectroscopy studies of graphite edges. Applied Surface Science 241, 43-48 (2005).

[6] Kobayashi, Y., Fukui, K.-i., Enoki, T., Kusakabe, K. \& Kaburagi, Y. Observation of zigzag and armchair edges of graphite using scanning tunneling microscopy and spectroscopy. Physical Review B 71, 193406 (2005).

[7] Takeda, K. \& Shiraishi, K. Theoretical possibility of stage corrugation in $\mathrm{Si}$ and Ge analogs of graphite. Physical Review B 50, 14916 (1994).

[8] Guzmán-Verri, G. G. \& Voon, L. C. L. Y. Electronic structure of silicon-based nanostructures. Physical Review B 76, 075131 (2007).

[9] Cahangirov, S., Topsakal, M., Aktürk, E., Şahin, H. \& Ciraci, S. Two-and one-dimensional honeycomb structures of silicon and germanium. Physical Review Letters 102, 236804 (2009).

[10] Liu, C.-C., Feng, W. \& Yao, Y. Quantum spin Hall effect in silicene and twodimensional germanium. Physical Review Letters 107, 076802 (2011).

[11] Kane, C. L. \& Mele, E. J. Quantum spin Hall effect in graphene. Physical Review Letters 95, 226801 (2005). 
[12] Aufray, B. et al. Graphene-like silicon nanoribbons on $\operatorname{Ag}(110)$ : A possible formation of silicene. Applied Physics Letters 96, 183102 (2010).

[13] De Padova, P. et al. Evidence of graphene-like electronic signature in silicene nanoribbons. Applied Physics Letters 96, 261905 (2010).

[14] Lalmi, B. et al. Epitaxial growth of a silicene sheet. Applied Physics Letters 97, 223109 (2010).

[15] Lin, C.-L. et al. Structure of silicene grown on $\operatorname{Ag}(111)$. Applied Physics Express 5, 045802 (2012).

[16] Fleurence, A. et al. Experimental evidence for epitaxial silicene on diboride thin films. Physical Review Letters 108, 245501 (2012).

[17] Vogt, P. et al. Silicene: compelling experimental evidence for graphenelike two-dimensional silicon. Physical Review Letters 108, 155501 (2012).

[18] Acun, A., Poelsema, B., Zandvliet, H. J. W. \& Van Gastel, R. The instability of silicene on $\operatorname{Ag}(111)$. Applied Physics Letters 103, 263119 (2013).

[19] Li, L. et al. Buckled germanene formation on Pt (111). Advanced Materials 26, 4820-4824 (2014).

[20] Dávila, M., Xian, L., Cahangirov, S., Rubio, A. \& Le Lay, G. Germanene: a novel two-dimensional germanium allotrope akin to graphene and silicene. New Journal of Physics 16, 095002 (2014).

[21] Bampoulis, P. et al. Germanene termination of ge ${ }_{2}$ pt crystals on $\mathrm{Ge}(110)$. Journal of Physics: Condensed matter 26, 442001 (2014).

[22] Derivaz, M. et al. Continuous germanene layer on $\mathrm{Al}(111)$. Nano Letters 15, 2510-2516 (2015).

[23] Švec, M. et al. Silicene versus two-dimensional ordered silicide: Atomic and electronic structure of $\mathrm{Si}-(19 \times 19) \mathrm{R} 23.4^{\circ} / \mathrm{Pt}(111)$. Physical Review B 89, 201412 (2014). 
[24] Safaei, A., Poelsema, B., Zandvliet, H. J. W. \& van Gastel, R. Spinodal decomposition driven formation of Pt-nanowires on $\mathrm{Ge}(001)$. New Journal of Physics 16, 113052 (2014).

[25] Garcia, J. C., de Lima, D. B., Assali, L. V. C. \& Justo, J. F. Group IV graphene-and graphane-like nanosheets. The Journal of Physical Chemistry C 115, 13242-13246 (2011).

[26] Xie, Q.-X. \& Nesper, R. Crystal structure of calcium zinc silicide, $\mathrm{CaZn}_{x} \mathrm{Si}_{2-x}(\mathrm{x}=0.80)$, and strontium germanium silicide, $\mathrm{SrGe}_{x} \mathrm{Si}_{2-x}(\mathrm{x}=$ 1.20). Zeitschrift für Kristallographie-New Crystal Structures 219, 95-96 (2004).

[27] Koskinen, P., Malola, S. \& Häkkinen, H. Self-passivating edge reconstructions of graphene. Physical Review Letters 101, 115502 (2008).

[28] Girit, Ç. Ö. et al. Graphene at the edge: stability and dynamics. Science 323, 1705-1708 (2009).

[29] Koskinen, P., Malola, S. \& Häkkinen, H. Evidence for graphene edges beyond zigzag and armchair. Physical Review B 80, 073401 (2009).

[30] Liu, M. et al. Quasi-freestanding monolayer heterostructure of graphene and hexagonal boron nitride on $\operatorname{Ir}(111)$ with a zigzag boundary. Nano letters 14, 6342-6347 (2014). 



\section{Synthesis of germanene on MoS $_{2}$}

To date germanene has only been synthesized on metallic substrates. A metallic substrate is usually detrimental for the two-dimensional Dirac nature of germanene because the important electronic states near the Fermi level of germanene can hybridize with the electronic states of the metallic substrate. Here we report the successful synthesis of germanene on molybdenum disulfide $\left(\mathrm{MoS}_{2}\right)$, a band gap material. Pre-existing defects in the $\mathrm{MoS}_{2}$ surface act as preferential nucleation sites for the germanene islands. The lattice constant of the germanene layer $(3.8 \pm$ $0.2 \AA$ ) is about $20 \%$ larger than the lattice constant of the $\mathrm{MoS}_{2}$ substrate ( $3.16 \AA$ ). Scanning tunneling spectroscopy measurements performed on the virtually continuous germanene layers reveal a $V$-shaped density of states, which is a clear hallmark of a two-dimensional Dirac material. These experimental results are in very good agreement with density functional theory calculations. 


\subsection{Introduction}

The discovery that graphene, a single layer of $s p^{2}$ hybridized carbon atoms arranged in a honeycomb registry, is stable, has resulted in numerous intriguing and exciting scientific breakthroughs. ${ }^{1-3}$ The electrons in graphene behave as relativistic massless fermions that are described by the Dirac equation, i.e. the relativistic variant of the Schrödinger equation. One might anticipate that elements with a similar electronic configuration, such as silicon ( $\mathrm{Si})$, germanium $(\mathrm{Ge})$ and tin $(\mathrm{Sn})$, also have a "graphene-like" allotrope. Unfortunately, silicene (the silicon analogue of graphene), germanene (the germanium analogue of graphene) and stanene (the tin analogue of graphene) have not been found in nature and therefore these twodimensional materials have to be synthesized. ${ }^{4-6}$ Theoretical calculations have revealed that the honeycomb lattices of the "graphene-like" allotropes of silicon and germanium are not fully planar, but slightly buckled. ${ }^{7,8}$ The honeycomb lattices of these two-dimensional materials consist of two triangular sub-lattices that are slightly displaced with respect to each other in a direction normal to the honeycomb lattice. Despite this buckling the two-dimensional Dirac nature of the electrons is predicted to be preserved. ${ }^{7,8}$ Another salient difference with graphene is that silicene and germanene have a substantially larger spin-orbit gap than graphene $(<0.05 \mathrm{meV}) .{ }^{9}$ Silicene's spin-orbit gap is predicted to be $1.55 \mathrm{meV}$, whereas the predicted spin-orbit gap of germanene is even $23.9 \mathrm{meV} .{ }^{10}$ This is very interesting because graphene and also silicene and germanene are in principle two-dimensional topological insulators and thus ideal candidates to exhibit the quantum spin Hall effect $^{11,12}$ The interior of a two-dimensional topological insulator exhibits a spinorbit gap, whereas topologically protected helical edge modes exist at the edges of the material. ${ }^{11-13}$ The two topologically protected spin-polarized edge modes have opposite propagation directions and therefore the charge conductance vanishes, whereas the spin conductance has a non-zero value.

In the past few years various groups have successfully synthesized silicene $e^{4,14-16}$ and germanene $e^{5,17-19}$ on a variety of substrates. To date germanene has only been grown on metallic substrates, such as $\mathrm{Pt}(111),{ }^{17} \mathrm{Au}(111),{ }^{5} \mathrm{Ge}_{2} \mathrm{Pt}^{18,20}$ and $\mathrm{Al}(111),{ }^{19}$ which might hinder a proper decoupling of the key electronic states of germanene near the Fermi level from the underlying substrate. Only Bampoulis et al. ${ }^{18}$ managed to resolve the primitive cell of the buckled honeycomb structure of germanene. 
Here we report the growth of germanene on a band gap material, namely $\mathrm{MoS}_{2}$. We found that the germanene layer, which is only weakly coupled to the $\mathrm{MoS}_{2}$ substrate, exhibits a clear V-shaped density of states, which is one of the hallmarks of a two-dimensional Dirac material. These experimental observations are in very good agreement with density functional theory calculations. The synthesis of germanene on $\mathrm{MoS}_{2}$ is a first step towards future germanene-based device applications.

\subsection{Experimental and calculation details}

The scanning tunneling microscopy (STM) and spectroscopy (STS) experiments have been performed at room temperature with an ultra-high vacuum STM (Omicron STM-1). The base pressure of the ultra-high vacuum system is $5 \times 10^{-11} \mathrm{mbar}$. We have used electrochemically etched tungsten STM tips. The $\mathrm{MoS}_{2}$ samples were freshly cleaved from synthesized $\mathrm{MoS}_{2}$ (acquired from 2D Semiconductors) and mounted on a Mo sample holder and subsequently introduced into the ultra-high vacuum system. Ge was deposited onto the $\mathrm{MoS}_{2}$ substrate by resistively heating a clean $\mathrm{Ge}(001)$ wafer that was located at a distance of $\sim 10 \mathrm{~mm}$ in front of the $\mathrm{MoS}_{2}$ substrate. Prior to the deposition the $\mathrm{Ge}(001)$ wafer was cleaned by a pre-anneal at $700 \mathrm{~K}$ for about $24 \mathrm{~h}$ followed by several cycles of argon ion bombardment at $800 \mathrm{eV}$ at room temperature and annealing at $1100 \mathrm{~K} .{ }^{21}$ After the deposition of germanium, the $\mathrm{MoS}_{2}$ sample was immediately inserted into the STM. The deposition rate was estimated by analyzing several STM images after the deposition of sub-monolayer amounts of germanium on the $\mathrm{MoS}_{2}$ substrate.

The density functional theory (DFT) calculations were performed using the projected augmented wave (PAW) formalism ${ }^{22}$ as implemented in the Vienna Ab Initio Simulation Package (VASP). ${ }^{23,24}$ Exchange-correlation effects were taken into account within the dispersion-corrected nonlocal optB88-vdW functional. ${ }^{25} \mathrm{An}$ energy cutoff of $600 \mathrm{eV}$ for the plane-waves and a convergence threshold of $10^{-5} \mathrm{eV}$ were employed. To avoid interactions between the supercells, a $25 \AA$ thick vacuum slab was added in the direction normal to the surface. The Brillouin zone was sampled by a $(4 \times 4) k$-point mesh. Structural relaxation was performed, while keeping the lowermost layer of sulfur atoms fixed, until the forces acting on the other atoms were less than $10^{-2} \mathrm{eV} / \AA$. 


\subsection{Results and discussion}

\subsection{1 $\mathrm{MoS}_{2}$}

$\mathrm{MoS}_{2}$ is a transition metal dichalcogenide. The elementary building block of a $\mathrm{MoS}_{2}$ crystal is a tri-layer structure consisting of one close-packed Mo atomic layer encapsulated between two atomic layers of close-packed $\mathrm{S}$ atoms. The atoms within this layer are strongly bonded by covalent interactions, whereas the interactions between the $\mathrm{MoS}_{2}$ layers are governed by weak van der Waals forces. The interlayer distance between $\mathrm{MoS}_{2}$ layer (S-Mo-S tri-layer) is approximately $0.65 \mathrm{~nm} . \mathrm{MoS}_{2}$ can easily be exfoliated and has a band gap that varies from an indirect $1.2 \mathrm{eV}$ to a direct $1.8 \mathrm{eV}$ for bulk and monolayer $\mathrm{MoS}_{2}$, respectively. ${ }^{26-29}$ Figure 6.1 shows the stick and ball model of $\mathrm{MoS}_{2}$ structure. In most cases only the S-atoms of $\mathrm{MoS}_{2}$

A

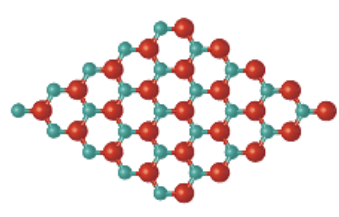

B

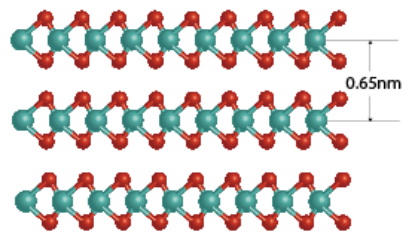

Figure 6.1: Ball stick model of $\mathrm{MoS}_{2}$ (A) Top view (B) side view
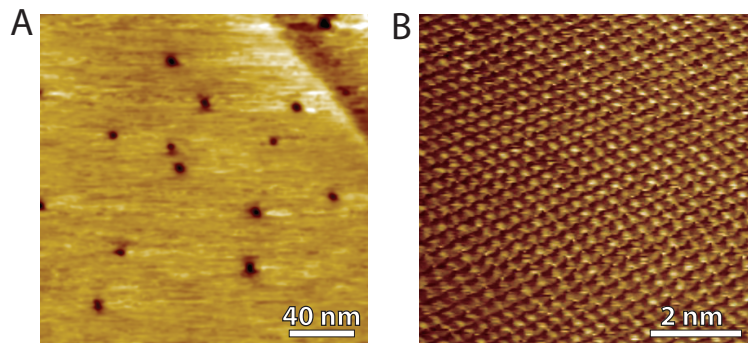

Figure 6.2: Scanning tunneling microscopy image of the $\mathrm{MoS}_{2}$ substrate (A) large scale with some defects (B) zoom-in of a defect free area 
substrate are resolved in STM images, resulting in a triangular structure with a lattice constant of $3.16 \AA$. The natural exfoliated $\mathrm{MoS}_{2}$ surface usually contains some defects (shown in Figure 6.2(A)) that are caused by a local variation in the stoichiometry, missing atoms or impurities. These defects can lead to n-type as well as p-type doping and are studied in depth. ${ }^{30-32}$ In addition, the defects affect the electronic structure of the $\mathrm{MoS}_{2}$ in the vicinity of the defects and therefore they appear usually substantially larger in STM images than they really are. In Figure 6.2(B) a high resolution, zoom-in, defect-free empty state STM image of the primitive honeycomb cell of $\mathrm{MoS}_{2}$ is shown.

\subsubsection{Germanene islands}

In Figure 6.3(A) a $\mathrm{MoS}_{2}$ substrate is shown after the deposition of $\sim 10 \%$ of a monolayer of germanium at room temperature (here one monolayer corresponds to a $(5 \times 5)$ cell of germanene on a $(6 \times 6)$ cell of the $\mathrm{MoS}_{2}$ substrate). Several germanium islands have been nucleated at pre-existing defects of the $\mathrm{MoS}_{2}$ substrate. From image to image the shape and size of these germanium islands changes, which implies (1) attachment and detachment of germanium atoms at the edges of the germanium islands and (2) rapid diffusion of germanium atoms across the surface. Interestingly, there is a hexagonal shaped vacancy island in the center of all the nucleated germanium islands.

Figure 6.3(B) shows a small scale STM image of the $\mathrm{MoS}_{2} /$ germanium substrate. The frizzy appearance of the germanium islands is clearly visible. We cannot exclude that part of this dynamics is induced by the scanning process. In the upper left part of the image a germanium island changes abruptly in size. In Figures 6.3(C) and (D) zoom-ins of a germanium-free part of the $\mathrm{MoS}_{2}$ substrate and a germanium island are shown, respectively. The lattice constant of the germanium islands is $20 \%$ larger than the lattice constant of the $\mathrm{MoS}_{2}$ substrate, i.e. $3.8 \pm 0.2 \AA$ for germanium versus $3.16 \AA$ for $\mathrm{MoS}_{2}$. The germanium islands exhibit a threefold symmetry as the underlying $\mathrm{MoS}_{2}$ substrate does. In addition, the high symmetry directions of the $\mathrm{MoS}_{2}$ substrate and the germanium islands are aligned (see Figure 6.3(A)).

Differential conductivity $(d I / d V)$ spectra recorded on a germanium island and the bare $\mathrm{MoS}_{2}$ substrate are depicted in Figure 6.3(E). As expected the $\mathrm{MoS}_{2}$ region has 

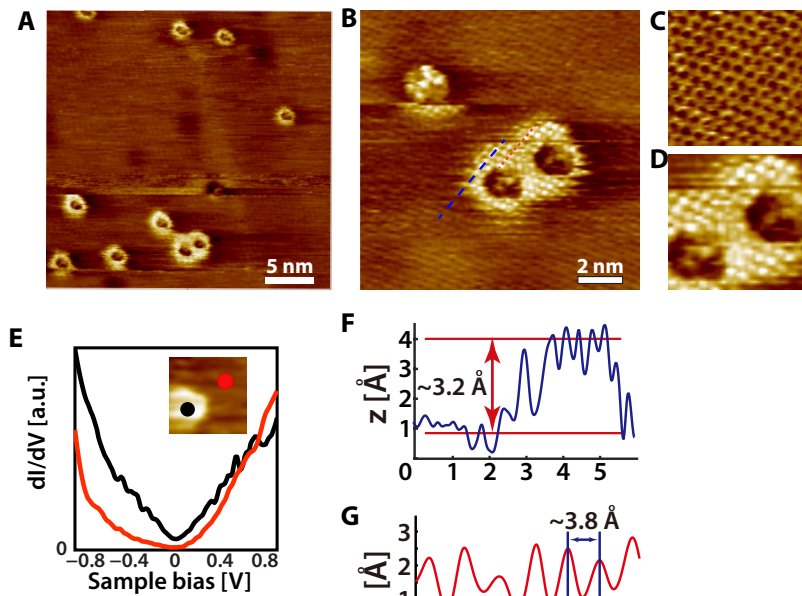

$\mathbf{F}$
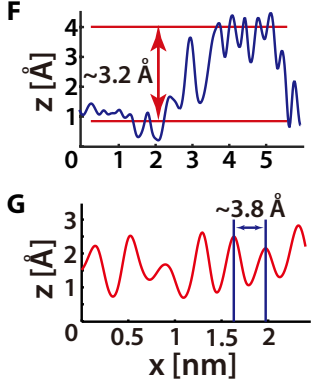

Figure 6.3: (A) Scanning tunneling microscopy image of the $\mathrm{MoS}_{2}$ surface after the deposition of $\sim 10 \%$ of a monolayer of germanium at room temperature. Small germanium islands are nucleated at pre-existing defect sites of the $\mathrm{MoS}_{2}$ substrate. The set points are $0.5 \mathrm{~V}$ and $0.6 \mathrm{nA}$.

(B) Scanning tunneling microscopy image of the $\mathrm{MoS}_{2} /$ germanium substrate. The sample bias is $0.5 \mathrm{~V}$ and the tunneling current is $0.3 \mathrm{nA}$.

(C) A zoom-in on a bare $\mathrm{MoS}_{2}$ area. The STM image reveals a honeycomb structure with a lattice constant of $3.15 \pm 0.2 \AA$, which corresponds to the lattice constant of $\mathrm{MoS}_{2}$. The sample bias and the tunneling current are the same as in (A).

(D) A zoom-in on the large germanene island of panel (B) reveals a hexagonal lattice with a lattice constant of $3.8 \pm 0.2 \AA$. The sample bias and the tunneling current are the same as in $(\mathrm{A})$.

(E) Differential conductivity recorded on a germanene island (black curve) and the surrounding $\mathrm{MoS}_{2}$ surface (red curve). The set points are $1 \mathrm{~V}$ and $0.3 \mathrm{nA}$.

(F) Line scan taken across the germanene island indicated with the blue dashed line in (B). The height of the germanene islands is $\sim 3.2 \AA$.

(G) Line scan taken on top of the germanene island indicated with the red dashed line in (B). The measured lattice constant of the germanene island corresponds to $3.8 \pm 0.2 \AA$.

a band gap, whereas the differential conductivity of the germanium island reveals a well-defined V-shape around zero bias $(|V|<0.5 \mathrm{~V})$. For small sample biases the differential conductivity is proportional to the density of states and therefore also 
the density of states of the germanium islands exhibits a V-shape. A V-shaped density of states is one of the hallmarks of a two-dimensional Dirac system. Based on these experimental observations we conclude that the germanium islands in Figure 6.3(A)-(B) are actually germanene islands.

In Figure 6.3(F)- $(\mathrm{G})$ line scans taken across the germanene island (see Figure 6.3(B) are depicted. The height of the germanene island is $\sim 3.2 \AA$. Since the electronic structure of germanene and $\mathrm{MoS}_{2}$ are substantially different it is not appropriate to compare this experimentally determined step height with theoretical predictions.

\subsubsection{Monolayer germanene}

In Figure 6.4(A) an STM image is shown after the deposition of $\sim 1$ monolayer of germanium. The germanene islands are now much larger and virtually the whole $\mathrm{MoS}_{2}$ surface is covered with germanene. The hexagonal shaped vacancy islands have all disappeared. The periodicity of the structure in Figure 6.4(A) is again 3.8 $\pm 0.2 \AA$. It is pointed out that the resolution in Figure 6.4(A) is somewhat lower
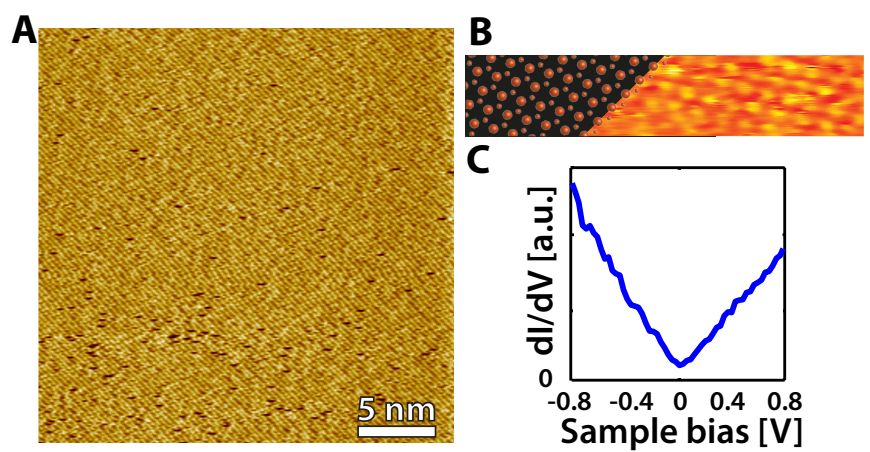

Figure 6.4: (A) A continuous film of germanene on $\mathrm{MoS}_{2}$ after the deposition of about a monolayer of germanium. The sample bias and tunnel current are $-1 \mathrm{~V}$ and $0.3 \mathrm{nA}$, respectively.

(B) Left: ball-and-stick model. Large (small) circles represent upward (downward) buckled germanium atoms. Right: High-resolution scanning tunneling microscopy image of germanene (only the upward buckled germanium atoms are resolved).

(C) Differential conductivity recorded on the germanene film. The set points are $-1.4 \mathrm{~V}$ and $0.6 \mathrm{nA}$, respectively. 


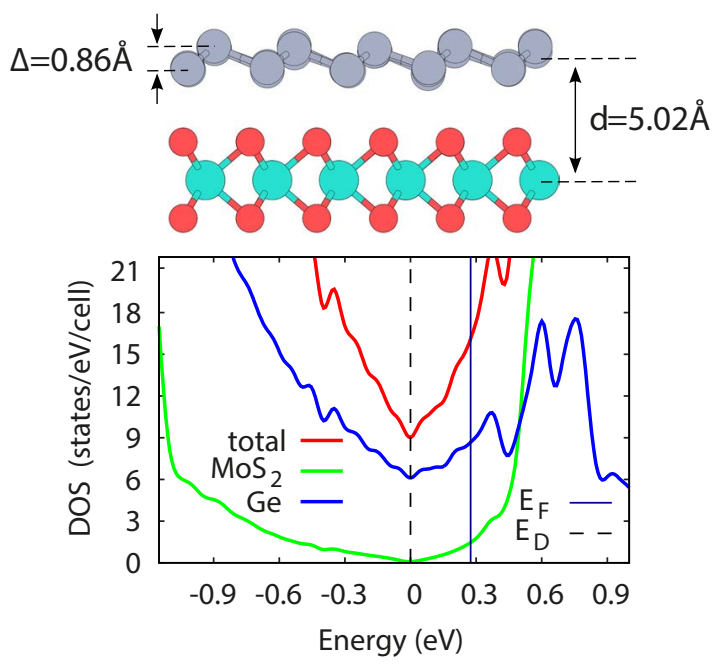

Figure 6.5: Calculated total and partial densities of states of germanene on $\mathrm{MoS}_{2}$. Vertical solid and dashed lines correspond to the Fermi energy and Dirac point, respectively.

than in the previous images. In Figure 6.4(A) only one of the two triangular sublattices is resolved. We did not find any evidence for the presence of a moiré pattern. Due to the $20 \%$ difference in lattice constants between germanene and $\mathrm{MoS}_{2}$ one expects only very small moiré periodicities $(<2 \mathrm{~nm}) .{ }^{33}$ As shown in Figure 6.4(C) the differential conductivity has a well-defined V-shape and thus confirms our earlier assignment that we are dealing with germanene. The differential conductivity does, however, not completely vanish at the Dirac point.

Free-standing low-buckled germanene is known to be a two-dimensional Dirac system that shares many properties with its famous counterpart graphene. ${ }^{34,35}$ However, here we are dealing with a germanene layer on a substrate and even though the interaction between germanene and $\mathrm{MoS}_{2}$ is rather weak, the electronic structure of germanene might be affected by the substrate.

\subsubsection{Density functional theory calculations}

To support our experimental observations of germanene, we have performed firstprinciples calculations using density functional theory (DFT). Unlike earlier DFT 
studies, where germanene has been considered on $\mathrm{MoS}_{2}$ in its free-standing configuration with a lattice constant of $3.97 \AA,{ }^{36,37}$ we consider here germanene laterally contracted by $\sim 5 \%$ in accordance with the experimental observations. Particularly, we use a $(5 \times 5)$ unit cell of germanene placed on top of a $(6 \times 6)$ unit cell of a $\mathrm{MoS}_{2}$ monolayer with a lattice constant $a_{\mathrm{MoS}_{2}}=3.18 \AA$, which yields a contracted germanene lattice constant $a_{G e}=3.82 \AA$. Subsequently we optimize the atomic structure by taking Van der Waals interactions into account and find an average equilibrium buckling parameter of germanene $\Delta=0.86 \pm 0.10 \AA$ and a corresponding interlayer distance $d=5.02 \AA$, defined as the distance between the in-plane averaged centers of mass of germanene and $\mathrm{MoS}_{2}$.

The density of states (DOS) is depicted in Figure 6.5 and shows a pronounced Vshape in the energy range of $\sim 1 \mathrm{eV}$ near the Fermi energy stemming from electronic states of germanium. Moreover, the shape of both germanene and $\mathrm{MoS}_{2} \mathrm{DOS}$ are found to be in very good agreement with the experimental $d I / d V$ spectra shown in Figure 6.3(E). Moreover, the non-zero DOS at the Dirac point observed in experimental spectra is also reproduced. The only difference concerns the position of

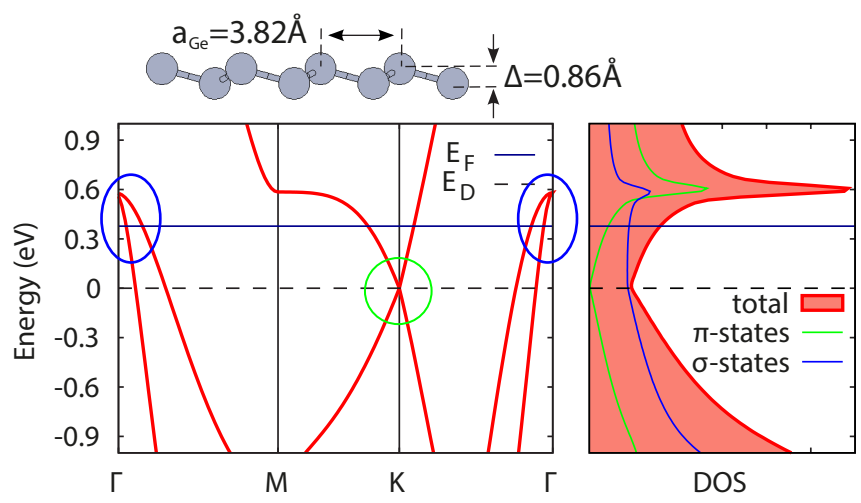

Figure 6.6: Band structure shown along the high-symmetry points of the Brillouin zone and the corresponding orbital-resolved density of states calculated for contracted germanene with a lattice constant $a_{G e}=3.82 \AA$ and a buckling parameter $\Delta=0.86 \pm 0.10 \AA$. Horizontal solid and dashed lines correspond to the Fermi energy and Dirac point, respectively. Green and blue circles mark the $\pi$ - and $\sigma$-bands, respectively. 

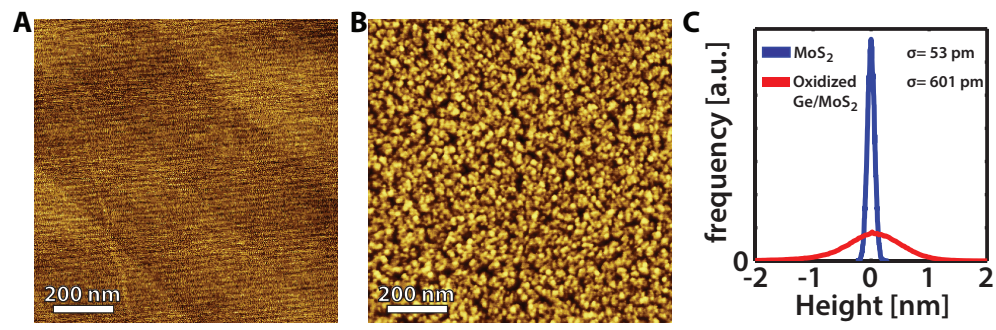

Figure 6.7: (A) Atomic force microscope image of $\mathrm{MoS}_{2}$ before Ge deposition, (B) Atomic force microscope image $\mathrm{MoS}_{2}$ after the Ge deposition. The AFM images are taken in ambient conditions,

(C) Probability distribution of the surface height of $\mathrm{MoS}_{2}$ and oxide $\mathrm{Ge} / \mathrm{MoS}_{2}$ measured with AFM

the Fermi energy, which is shifted in the calculated DOS toward higher energies by $\sim 0.3 \mathrm{eV}$. This shift indicates an $n$-type doping of the system. The absence of such a shift in the experimental spectra can be associated with the presence of acceptor impurities (e.g., O) or unsaturated defects in the sample. To gain more insight into the origin of the finite DOS at the Dirac point as well as the observed doping, we calculate the band structure and perform orbital analysis of contracted free-standing germanene with the above mentioned structural parameters $a_{G e}$ and $\Delta$. The results are shown in Figure 6.6. In addition to the $\pi$-bands leading to the formation of a Dirac cone in the vicinity of the K-point, there are two $\sigma$-bands close to the $\Gamma$ point. The emergence of those bands is related to the relatively large buckling of germanene, which makes the occupation of $\sigma$-states energetically less favorable ${ }^{35}$ and leads to a $\sigma-\pi$ charge transfer. As a consequence of this process, the Fermi energy is shifted relative to the Dirac point, whereas unoccupied $\sigma$-bands produce a finite DOS in the relevant energy region. The $\sigma$-states contribution to the DOS is weakly dependent on energy as the corresponding dispersion is nearly quadratic $\left(E_{\sigma}(k) \sim k^{2}\right)$. Therefore, we conclude that the observed V-shape of the total DOS originates predominantly from the $\pi$-states of germanene. 


\subsubsection{Ambient experiments}

After having performed the STM and STS measurements we took the sample (germanene on $\mathrm{MoS}_{2}$ ) out of the ultra-high vacuum system for an ex-situ analysis. Xray photoelectron spectroscopy measurements revealed that the germanene was oxidized. The sample also was investigated by atomic force microscope (AFM) before and after Ge deposition. Figure 6.7(A) shows the bare $\mathrm{MoS}_{2}$ sample with smooth surface. After deposition of Ge in UHV, the sample was taken out of UHV imaged by AFM (see Figure 6.7(B)). The image in Figure 6.7(B) is rougher than in Figure 6.7(A) indicating that the Ge has been oxidized.

\subsection{Conclusions}

In summary, we have synthesized large continuous layers of germanene on a band gap material. The germanene islands preferentially nucleate at pre-existing defects of the $\mathrm{MoS}_{2}$ surface. Germanene's lattice constant is about 20\% larger than that of $\mathrm{MoS}_{2}$ and the angle between the two lattices is 0 degrees. The density of states of the germanene layer exhibits a well-defined V-shape around the Fermi level, which is one of hallmarks of a two-dimensional Dirac system. These experimental observations are in very good agreement with state-of-the-art DFT calculations. 


\section{Bibliography}

[1] Novoselov, K. S. et al. Electric field effect in atomically thin carbon films. Science 306, 666-669 (2004).

[2] Novoselov, K. S. et al. Two-dimensional gas of massless dirac fermions in graphene. Nature 438, 197-200 (2005).

[3] Geim, A. K. \& Novoselov, K. S. The rise of graphene. Nature Materials 6, 183-191 (2007).

[4] Vogt, P. et al. Silicene: compelling experimental evidence for graphenelike two-dimensional silicon. Physical Review Letters 108, 155501 (2012).

[5] Dávila, M., Xian, L., Cahangirov, S., Rubio, A. \& Le Lay, G. Germanene: a novel two-dimensional germanium allotrope akin to graphene and silicene. New Journal of Physics 16, 095002 (2014).

[6] Zhu, F.-f. et al. Epitaxial growth of two-dimensional stanene. Nature Materials 14, 1020-1025 (2015).

[7] Guzmán-Verri, G. G. \& Voon, L. C. L. Y. Electronic structure of silicon-based nanostructures. Physical Review B 76, 075131 (2007).

[8] Cahangirov, S., Topsakal, M., Aktürk, E., Şahin, H. \& Ciraci, S. Two-and one-dimensional honeycomb structures of silicon and germanium. Physical Review Letters 102, 236804 (2009).

[9] Yao, Y., Ye, F., Qi, X.-L., Zhang, S.-C. \& Fang, Z. Spin-orbit gap of graphene: First-principles calculations. Physical Review B 75, 041401 (2007).

[10] Liu, C.-C., Feng, W. \& Yao, Y. Quantum spin Hall effect in silicene and twodimensional germanium. Physical Review Letters 107, 076802 (2011).

[11] Kane, C. L. \& Mele, E. J. $Z_{2}$ topological order and the quantum spin Hall effect. Physical Review Letters 95, 146802 (2005).

[12] Kane, C. L. \& Mele, E. J. Quantum spin Hall effect in graphene. Physical Review Letters 95, 226801 (2005). 
[13] Bernevig, B. A. \& Zhang, S.-C. Quantum spin Hall effect. Physical Review Letters 96, 106802 (2006).

[14] Fleurence, A. et al. Experimental evidence for epitaxial silicene on diboride thin films. Physical Review Letters 108, 245501 (2012).

[15] Chiappe, D. et al. Two-dimensional Si nanosheets with local hexagonal structure on a $\mathrm{MoS}_{2}$ surface. Advanced Materials 26, 2096-2101 (2014).

[16] Feng, B. et al. Evidence of silicene in honeycomb structures of silicon on $\operatorname{Ag}(111)$. Nano Letters 12, 3507-3511 (2012).

[17] Li, L. et al. Buckled germanene formation on Pt (111). Advanced Materials 26, 4820-4824 (2014).

[18] Bampoulis, P. et al. Germanene termination of $\mathrm{Ge}_{2} \mathrm{Pt}$ crystals on $\mathrm{Ge}(110)$. Journal of Physics: Condensed Matter 26, 442001 (2014).

[19] Derivaz, M. et al. Continuous germanene layer on $\mathrm{Al}(111)$. Nano Letters 15, 2510-2516 (2015).

[20] Zhang, L., Bampoulis, P., van Houselt, A. \& Zandvliet, H. J. W. Twodimensional Dirac signature of germanene. Applied Physics Letters 107, 111605 (2015).

[21] Zandvliet, H. J. W. The Ge(001) surface. Physics Reports 388, 1-40 (2003).

[22] Blöchl, P. E. Projector augmented-wave method. Physical Review B 50, 17953 (1994).

[23] Kresse, G. \& Futhmüller, J. Efficient iterative schemes for ab initio totalenergy calculations using a plane-wave basis set. Physical Review B 54, 11169 (1996).

[24] Kresse, G. \& Joubert, D. From ultrasoft pseudopotentials to the projector augmented-wave method. Physical Review B 59, 1758 (1999).

[25] Klimeš, J., Bowler, D. R. \& Michaelides, A. Van der Waals density functionals applied to solids. Physical Review B 83, 195131 (2011). 
[26] Mattheiss, L. Band structures of transition-metal-dichalcogenide layer compounds. Physical Review B 8, 3719 (1973).

[27] Böker, T. et al. Band structure of $\mathrm{MoS}_{2}, \mathrm{MoSe}_{2}$, and $\alpha-\mathrm{MoTe}_{2}$ : Angleresolved photoelectron spectroscopy and ab initio calculations. Physical Review B 64, 235305 (2001).

[28] Mak, K. F., Lee, C., Hone, J., Shan, J. \& Heinz, T. F. Atomically thin $\mathrm{MoS}_{2}$ : a new direct-gap semiconductor. Physical Review Letters 105, 136805 (2010).

[29] Splendiani, A. et al. Emerging photoluminescence in monolayer $\mathrm{MoS}_{2}$. Nano Letters 10, 1271-1275 (2010).

[30] Whangbo, M.-H. et al. On the correlation between the scanning tunneling microscopy image imperfections and point defects of layered chalcogenides 2H-MX $2(\mathrm{M}=\mathrm{Mo}, \mathrm{W} ; \mathrm{X}=\mathrm{S}, \mathrm{Se})$. Surface Science 326, 311-326 (1995).

[31] Addou, R., Colombo, L. \& Wallace, R. M. Surface defects on natural $\mathrm{MoS}_{2}$. ACS Applied Materials \& Interfaces 7, 11921-11929 (2015).

[32] Lin, J., Pantelides, S. T. \& Zhou, W. Vacancy-induced formation and growth of inversion domains in transition-metal dichalcogenide monolayer. ACS Nano 9, 5189-5197 (2015).

[33] Yankowitz, M. et al. Emergence of superlattice Dirac points in graphene on hexagonal boron nitride. Nature Physics 8, 382-386 (2012).

[34] Neto, A. H. C., Guinea, F., Peres, N. M. R., Novoselov, K. S. \& Geim, A. K. The electronic properties of graphene. Reviews of Modern Physics 81, 109 (2009).

[35] Acun, A. et al. Germanene: the germanium analogue of graphene. Journal of Physics: Condensed Matter 27, 443002 (2015).

[36] Li, X., Wu, S., Zhou, S. \& Zhu, Z. Structural and electronic properties of germanene/ $\mathrm{MoS}_{2}$ monolayer and silicene/ $\mathrm{MoS}_{2}$ monolayer superlattices. Nanoscale Research Letters 9, 1-9 (2014). 
[37] Li, X. D., Wu, S. Q. \& Zhu, Z. Z. Band gap control and transformation of monolayer- $\mathrm{MoS}_{2}$-based hetero-bilayers. Journal of Materials Chemistry C 3, 9403-9411 (2015). 



\section{Summary}

The research of low-dimensional electron systems has received quite some attention during the last two decades. This thesis deals with the synthesis and characterization of metal-induced nanowires on $\mathrm{Ge}(110)$ surface and germanene, a novel two-dimensional material, which can be considered as the germanium analogue of graphene. Experiments of Au- and Pt- induced nanowires on $\mathrm{Ge}(110)$ are presented in this thesis. Inspired by the Pt/Ge system we have exploited a novel way to synthesize germanene by using the formation of the eutectic phase. This eutectic phase is composed of $22 \% \mathrm{Pt}$ and $78 \% \mathrm{Ge}$. Upon cooling the eutectic undergoes spinodal decomposition into a $\mathrm{Ge}_{2} \mathrm{Pt}$ and a pure Ge phase. Furthermore, the growth of germanene on a band gap material $-\mathrm{MoS}_{2}$ - has been presented in the final part of this thesis. In chapter 1, a brief introduction of the low-dimensional electron systems has been given. The experimental details have been addressed in chapter 2 .

In chapter 3, we have demonstrated the properties of the bare Ge(110) surface and Au-induced nanowires. After several cycles of cleaning the bare Ge(110) surface displayed several reconstructions, which are all composed of pentagons i.e. fivemembered atom rings. In our case, there are three different reconstruction domains i.e. $16 \times 2, c(8 \times 10)$ and a disordered phase. Three well-defined electronic states are extracted from the normalized differential conductive spectrum. Two of the states, located near the Fermi level, respectively $(0.4 \mathrm{eV}$ and $-0.3 \mathrm{eV})$, are related to the pentagons. The third state has been tentatively ascribed to the Ge-Ge back bond. Subsequently, we have studied the structural and electronic properties of $\mathrm{Ge}(110)$ after the deposition of a sub-monolayer amount of Au. Upon Au deposition and annealing nanowires are formed that are aligned along the 'ridges' of the unreconstructed $\mathrm{Ge}(110)$ surface (this direction is referred to as the [110] direction). Scanning tunneling spectroscopy measurements recorded on these nanowires reveal that the nanowires are semiconducting, suggesting that the nanowires are composite of Ge. 
In chapter 4, we have studied the structural and electronic properties of Pt-induced nanowires on $\mathrm{Ge}(110)$ surfaces by scanning tunneling microscopy and low energy electron microscopy. The deposition of a sub-monolayer amount of $\mathrm{Pt}$ and subsequent annealing at $1100( \pm 25) \mathrm{K}$ results into nanowires which are aligned along the densely packed [110] direction of the Ge(110) surface. With increasing Pt coverage the nanowires form densely packed arrays with separations of $1.1 \pm 0.1 \mathrm{~nm}$, $2.0 \pm 0.1 \mathrm{~nm}$, and $3.4 \pm 0.1 \mathrm{~nm}$. Ge pentagons reside in the troughs for nanowire separations of $3.4 \mathrm{~nm}$, however, for smaller nanowire separations no pentagons are found. Spatially resolved scanning tunneling spectroscopy measurements reveal a filled electronic state at $-0.35 \mathrm{eV}$. This electronic state is present in the troughs as well as on the nanowires. The $-0.35 \mathrm{eV}$ state has the strongest intensity on the pentagons, which is in line with the results of the bare Ge(110) surface as discussed in the previous chapter. For Pt depositions exceeding two monolayers, pentagonfree nanowire patches are found, that coexist with Pt-Ge clusters. Upon annealing at $1050 \mathrm{~K}$ these Pt-Ge clusters become liquid-like, indicating that we are dealing with eutectic $\mathrm{Pt}_{0.22} \mathrm{Ge}_{0.78}$ clusters. Low energy electron microscopy videos reveal the formation and spinodal decomposition of these eutectic Pt-Ge clusters.

In chapter 5, we suggest that the Pt-Ge clusters emerged in the previous chapter, are actually $\mathrm{Ge}_{2} \mathrm{Pt}$ crystallites. The outermost of the crystalline exhibit a honeycomb structure with a nearest-neighbor distance of $2.5 \pm 0.1 \AA$. This honeycomb lattice is composed of two hexagonal sub-lattices that are displaced vertically by $0.2 \AA$ with respect to each other. The interior of the germanene sheets displays a V-shaped density of states indicative of a 2D Dirac system. Based on our experimental observations we propose that the outermost layer of the Pt-Ge crystals is a germanene layer. However, the density of states at the minimum of the V-shaped curve does not completely vanish, which we ascribe to the underlying metallic Pt-Ge substrate. Two types of step edges are found on the germanene sheets: straight reconstructed zigzag step edges with a $4 \times$ periodicity and rough step edges. Both types of step edges have a parabolic shaped $d I / d V$ curves.

In chapter 6, we have synthesized germanene on a band gap material: $\mathrm{MoS}_{2}$ and investigated the electronic properties of germanene. The germanene islands preferentially nucleate at pre-existing defects of the $\mathrm{MoS}_{2}$ surface. Germanene's lattice constant is about 20\% larger than that of $\mathrm{MoS}_{2}$ and the angle between the two lattices is 0 degrees. The density of states of the germanene layer exhibits a well-defined 
V-shape around the Fermi level, which is one of the hallmarks of a two-dimensional Dirac system. These experimental observations are in very good agreement with state-of-the-art DFT calculations. 



\section{Samenvatting}

Het onderzoek naar laag dimensionale elektronsystemen heeft in de laatste tien jaren veel aandacht gekregen. Dit proefschrift beschrijft zowel de groei en karakterisatie van metaal geïnduceerde één dimensionale nanodraden gegroeid op een $\mathrm{Ge}(110)$ oppervlak en germanene, een nieuw tweedimensionaal materiaal, welke kan worden beschouwd als een germanium equivalent aan graphene. In het geval van de nanodraden worden zowel Au als Pt geïnduceerde draden beschreven. Geïnspireerd door de methode gebaseerd op Pt-Ge, hebben we een nieuwe manier gevonden om germanene te synthetiseren door gebruik te maken van de eutectische menging. De eutectische fase bestaat voor $22 \%$ uit Pt en voor $78 \%$ uit Ge. Tijdens het koelen ondergaat het eutectische mengsel een ontleding in een $\mathrm{Ge}_{2} \mathrm{Pt}$ en een pure Ge fase. Verder wordt in dit proefschrift de groei van germanene op een halfgeleider, $\mathrm{MoS}_{2}$, beschreven. Het hoofdstuk 1 zal laag dimensionale elektronsystemen introduceren. In hoofdstuk 2 volgt de experimentele beschrijving.

In hoofdstuk 3 worden de eigenschappen van een schoon $\mathrm{Ge}(110)$ oppervlak beschreven als wel de eigenschappen van het zelfde oppervlak met Au-geïnduceerde nanodraden. $\mathrm{Na}$ een aantal schoonmaakcycli vertoond het $\mathrm{Ge}(110)$ oppervlak verscheidene oppervlaktereconstructies. Deze reconstructies bestaan allen uit atoomgroepjes welke zijn geordend in vijfhoeken. In ons geval zijn er drie reconstructies te vinden, namelijk $16 \times 2, c(8 \times 10)$ en een ongeordende fase. Uit het genormaliseerde afgeleide geleidingsspectrum zijn ook drie goed te onderscheiden toestanden gevonden. Twee van deze toestanden, welke rond het Ferminiveau liggen ( $0.4 \mathrm{eV}$ en -0.3 $\mathrm{eV})$, zijn gerelateerd aan de zojuist beschreven vijfhoeken. De derde toestand wordt voorlopig toegekend aan een Ge-Ge verbinding. Vervolgens hebben we de structurele en de elektronische eigenschappen van het $\mathrm{Ge}(110)$ oppervlak bestudeerd na het opdampen van een sub-monolaag aan Au. Na het opdampen van Au en het vervolgens temperen zijn nanodraden te vinden welke zich gelijkrichten aan de stapranden in de zogenoemde [110] richting. Raster tunnel spectroscopie metingen 
van de draden tonen aan dat deze draden een halfgeleider eigenschap hebben, wat waarschijnlijk betekent dat de draden uit Ge bestaan.

In hoofdstuk 4 hebben we met raster tunnel microscopie en lage energie elektronenmicroscopie de structuur en elektronische eigenschappen van Pt-geïnduceerde nanodraden op een $\mathrm{Ge}(110)$ oppervlak gemeten. Het opdampen van een sub-monolaag aan Pt gevolgd door temperen op een temperatuur van $1100 \mathrm{~K}$ resulteerde in een zeer hoge dichtheid aan nanodraden welke parallel liggen in de [11̄0] richting. Bij hogere Pt bedekking vormen de nanodraden een bedekking waarbij de draden van elkaar gescheiden zijn met een afstand van $1.1 \mathrm{~nm}, 2.0 \mathrm{~nm}$ of $3.4 \mathrm{~nm}$. Ge vijfhoeken liggen enkel in het dal behorend tot de nanodraden met een scheiding van $3.4 \mathrm{~nm}$. Scans van het oppervlak laten zien dat er een gevulde toestand is bij een voltage van $-0.35 \mathrm{eV}$. Deze toestand is zowel aanwezig in het dal tussen als op de nanodraden. De toestand is het sterkst vertegenwoordigd bij metingen op de vijfhoeken, wat overeenkomt met de vindingen op schoon $\mathrm{Ge}(110)$. Bij het opdampen van meer dan twee monolagen aan Pt zijn er nanodraden te vinden welke geen vijfhoeken bevatten en welke gepaard gaan met Pt-Ge clusters. Bij het verhitten tot $1040 \mathrm{~K}$ worden deze clusters vloeibaar, wat aantoont dat we hier te mkaen hebben met eutectische $\mathrm{Pt}_{0.22} \mathrm{Ge}_{0.78}$ clusters. Lage energie elektronenmicroscopie kan dit proces vastleggen.

In hoofdstuk 5 suggereren we dat de clusters bestaan uit kristallijn $\mathrm{Ge}_{2} \mathrm{Pt}$. De toplaag van deze kristallen bestaat uit een honingraatstructuur waarbij de naaste atomen gescheiden zijn door een afstand van $2.5 \AA$ A . Deze honingraat bestaat uit twee hexagonale sub-roosters welke verticaal van elkaar gescheiden zijn met $0.2 \AA$. Binnenin deze honingraat, welke als germanene wordt verondersteld, vertoond een toestandsdichtheid in de vorm van een V. Deze V-vorm is een eigenschap van 2D Dirac materialen. Gebaseerd op onze experimentele ondervindingen stellen wij dat de buitenste laag van de Pt-Ge kristallen een germanene laag is. Wat wel gevonden is, is dat het minimum van de toestandsdichtheid niet nul wordt. Dit schrijven wij toe aan interactie tussen de germanene laag en het onderliggende Pt-Ge substraat. Twee soorten stapranden zijn gevonden op de germanene vellen: rechte zigzag stapranden met een $4 \times$ periodiciteit en ruwe stapranden. Beide soorten stapranden laten een parabolische $d I / d V$ curve zien.

In hoofdstuk 6 beschrijven we de groei van germanene op een halfgeleider: $\mathrm{MoS}_{2}$ en bestuderen we de elektronische eigenschappen van germanene. De germanene 
eilanden vormen op de al aanwezige defecten in het oppervlak van $\mathrm{MoS}_{2}$. De roosterafstand van germanene is ongeveer $20 \%$ groter dan dat van $\mathrm{MoS}_{2}$ en de roosters zijn niet gedraaid ten opzichte van elkaar. De toestandsdichtheid van de germanene laag toont een duidelijke V-vorm rond het Ferminiveau, wat een kenmerk is van een tweedimensionaal Dirac materiaal. De experimenten zijn in goede overeenstemming met hedendaagse DFT berekeningen. 



\section{Publications}

- L.Zhang, P. Bampoulis, A.N. Rudenko, Q. Yao, A. van Houselt, B. Poelsema, M.I. Katsnelson and H.J.W. Zandvliet, Structural and electronic properties of Germanene on $\mathrm{MoS}_{2}$, Physical Review Letters 116, 256804 (2016), featured in Physics.

- L. Zhang, P. Bampoulis, A. Safaei, H.J.W. Zandvliet and A. van Houselt, Structural and electronic properties of Pt induced nanowires on Ge(110), Applied Surface Science 387, 766-770 (2016).

- L.Zhang, P. Bampoulis, A. van Houselt and H.J.W. Zandvliet, Two-dimensional Dirac signature of germanene, Applied Physics Letters 107, 111605 (2015).

- A. Acun, L. Zhang, P. Bampoulis, M. Farmanbar, M. Lingenfelder, A. van Houselt, A.N. Rudenko, G. Brocks, B. Poelsema, M.I. Katsnelson and H.J.W. Zandvliet, Germanene: the germanium analogue of graphene, Journal Physics Condensed Matter 27, 443002 (2015).

- P. Bampoulis, L. Zhang, A. Safaei, R. van Gastel B. Poelsema and H.J.W. Zandvliet, Germanene termination of $\mathrm{Ge}_{2} \mathrm{Pt}$ crystals on $\mathrm{Ge}(110)$, Journal of Physics Condensed Matter, 26, 442001 (2014).

- C.J. Walhout, A. Acun, L. Zhang, M. Ezawa and H.J.W. Zandvliet, Scanning tunnelling spectroscopy study of the Dirac spectrum of germanene, Journal of Physics Condensed Matter 28, 284006 (2016). 
- P. Bampoulis, A. Acun, L. Zhang and H.J.W. Zandvliet, Electronic and energetic properties of Ge(110) pentagons, Surface Science 626, 1 (2014).

- Q. Yao, R. van Bremen, G.J. Slotman, L. Zhang, S. Haartsen, K. Sotthewes, P. Bampoulis, P.L. de Boeij, A. van Houselt, S. Yuan and H.J.W. Zandvliet, Spatially resolved electronic structure of twisted graphene, Physical Review B 95, 245116 (2017) 


\section{Acknowledgements}

In the last four years, I have received countless help and support from many people. Without their precious support, it would have not been possible to conduct this research and obtain the doctoral degree smoothly.

Firstly, I would like to express my sincere thanks to my supervisor Prof. Harold Zandvliet. Harold, thanks for giving me the opportunity to join the Physics of Interface and Nanomaterials (PIN) group. Thanks for your continuous support, encouragement, and the help you have provided throughout my Ph.D. study. Your immense knowledge, patience, and considerate behavior impressed me a lot. Your guidance helped me in all the time of research and writing of this thesis. I could not have imagined having a better supervisor for my Ph.D. study.

I would like to thank Dr. Arie van Houselt as my co-supervisor and the help he has provided during the process of my Ph.D. My sincere thanks also go to the scientific staff of the group Prof. Bene Poelsema, Dr. Stefan Kooij, and Dr. Herbert Wormeester who have provided me with valuable discussions, suggestions, and advice during the last four years. The technical staff of the group: Martin, Hans, and Herman thanks for the countless number of times you helped me fixing the STM or the UHV system. I would like to thank Simone, the secretary of the group, for her support and handling of many administrative tasks in the past four years.

Prof. B. Poelsema, Prof. M. Huijben, Prof. G. Palasantzas and Prof. N. Oncel: I would like to thank you for agreeing to be part of my graduation committee and thoroughly reviewing my thesis.

I would like to acknowledge the China Scholarship Council for the financial support of this research.

I thank my fellow group members for the scientific and non-scientific discussions, and for all the fun we have had in the last four years. I acknowledge Pantelis and Qirong to be my paranymphs.

Pantelis, thanks for all the interactions we had these years. You know how much 
you have contributed to my work. I really appreciated the numerous conversations we had. It was a nice experience to attend the conference with you in the UK. Adil, you were always happy to provide me with help. Thanks for your assistance to make me feel comfortable as a new member of the group. Qirong, my fellow country man, I never felt alone when you became a colleague of mine. Thanks for so many things. Kai, I appreciate the many very valuable talks we had, which made me think deeper and feel better. Rik, I am happy to talk with you in the lab. By the way thanks for the Dutch translation of the summary of my thesis! Nikolai, thanks for the collaboration and the lots of fun we have in every summer. Edwin, Wojtek, Mikhail, Özlem, Floor, Carolien, Jordi, and all other BSc and MSc students of the PIN group thanks for all the moments we have shared. I would also like to thank the former group members especially Hairong and Ali for the support and help they have provided. My special thanks go to Hairong for picking me up in the station and the considerable help afterwards.

My life become colorful with the friends I have made in Twente. Shanqiu, Qingqian, and Aijie we started our Ph.D. at almost the same time got to know each other at the beginning and became good friends. I appreciate this friendship. And we made a lot of fun together with Yanbo and Jie at the first year of our Ph.D. I miss those days! I would also like to thank Chuan, Johnny, Mengjiao, Haishan, Jianfeng, Huanshu, Haichuan, Peng, Yuxing, Minmin, and many more, for all the fun we have had.

Last but not the least, I would like to thank the continuous unconditional support from my father, my sisters and my brother. I cannot find any words to express my emotions to them. I would like to thank my parents-in-law for their spiritual support. My deepest gratitude goes to my wife Aimei and my lovely new-born daughter Aojia, I love you from the bottom of my heart. 